\title{
International Coordination of Macroprudential Policies with Capital Flows and Financial Asymmetries
}

\author{
William Chen* Gregory Phelan ${ }^{\dagger}$
}

This version: June 2, 2020

\begin{abstract}
Lack of coordination for prudential regulation hurts developing economies but benefits advanced economies. We consider a two-country macro model in which countries have limited ability to issue state-contingent contracts in international markets. Both countries have incentives to stabilize their economy by using prudential limits, but the emerging economy depends on the advanced economy to bear global risk. Intermediating global risk requires bearing systemic risk, which financially developed economies are unwilling to bear, preferring financial stability over credit flows. Advanced economies prefer tighter prudential limits than would occur with coordination, to the harm of emerging economies.
\end{abstract}

Keywords: International Capital Flows, Capital Controls, Macroeconomic Instability, Macroprudential Regulation, Policy Coordination, Spillovers, Financial Crises.

JEL classification: E44, F36, F38, F42, G15.

\footnotetext{
*Williams College. Email: wyc1@ williams.edu

${ }^{\dagger}$ Department of Economics, Williams College. Email: gregory.phelan@gmail.com.

We are grateful for feedback from Patrick Conway, Paolo Pesenti, Stan Rabinovich, Eli Remolona, Alp Simsek, and James Yetman.
} 


\section{Introduction}

Following the Great Recession, economists and policy makers have debated the appropriate uses of prudential regulations to promote financial and macroeconomic stability. ${ }_{1}^{1}$ Many hope that limiting risk-taking can decrease the frequency and duration of times of economic or financial stress. The effectiveness and implementation of these policies are complicated by interactions through international capital markets as well as the degree of international coordination. For these reasons, international coordination of prudential regulations have garnered renewed interest (Basel III was created in response to the financial crisis). This paper considers how the asymmetric nature of the global financial system is likely to affect policy coordination.

Economists have long recognized the crucial role of financial development to facilitate global intermediation ${ }^{2}$ Advanced economies (AEs) like the U.S. act as the "world's global banker," providing safe debt to emerging market economies (EMEs) and investing in risky foreign assets. For AEs to bear global risk on behalf of EMEs requires not only bearing fundamental risk but also exposing themselves to long-run financial instability; the frequency or duration of crises for AEs may be higher than would occur if AEs did not have leveraged aggregate balance sheets. While fundamental risk is generally compensated by excess returns (Gourinchas et al., 2010), with financial frictions or incomplete markets, sources of long-run instability need not be correctly compensated by returns (i.e., pecuniary externalities). Stability necessitates prudential policies.

In light of these concerns, our paper theoretically considers how global spillovers through international capital markets can affect countries' capital account policy choices. We use a stochastic macroeconomic model based off Brunnermeier and Sannikov (2015). Two countries (AE and EME) produce two goods and have limited ability to issue equity in international markets. The financial asymmetry is that the AE country has lower risk aversion than the EME country (see Gourinchas et al., 2010). There are no trade frictions other than (potentially) regulations imposed by each country limiting capital inflows or domestic leverage. Because international financial mar-

\footnotetext{
${ }^{1}$ Common prudential policies include bank capital requirements, counterparty concentration limits, interbank exposure limits, loan-to-value ratios, and reserve requirements, as well as limitations on capital inflows.

${ }^{2}$ See for example Gourinchas and Rey (2007), Gourinchas et al. (2010), Lane and Milesi-Ferretti (2007), and Maggiori (2017).
} 
kets are imperfect, global output depends endogenously on the relative share of wealth in each country, and debt imbalances (i.e., capital inflows or leverage) create volatility that increases the fraction of the time the global economy spends with misallocated capital. Higher leverage and capital inflows lead to a better static allocation of capital and higher growth at the cost of increased volatility that hurts dynamic global stability. A social planner would impose prudential controls in both the $\mathrm{AE}$ and the EME because countries use too much leverage in the laissez-faire competitive equilibrium.

The asymmetric role of bearing global financial instability creates different incentives for AEs and EMEs to use prudential policies. Advanced economies have incentives to impose marginally tighter prudential regulations to "regulate away" sources of long-run financial instability (decreasing the frequency and duration of crises), which benefits AEs at the expense of EMEs. In contrast, EMEs want AEs to provide intermediation because AEs have superior risk-bearing capacities. Policy coordination must balance these opposing incentives. We show it is unlikely that advanced economies are willing to expose themselves to long-run financial instability on behalf of EMEs.

We first consider how regulation in one country positively affects outcomes and aggregate dynamics in the other (what we call "macro-level spillovers"). We find that tighter prudential regulations in the $\mathrm{AE}$ will decrease global volatility when the AE is relatively poor and improve the terms of trade for the $\mathrm{AE}$ when the $A E$ is relatively poor. As a result, tighter regulations will decrease the frequency with which the $\mathrm{AE}$ is relatively poor. Hence, prudential controls by the $\mathrm{AE}$ increase the frequency with which the EME is relatively poor. Accordingly, the EME has strategic incentives to also tighten its policy in order to increase the frequency of being relatively rich. Incomplete markets due to limited equity issuance are crucial for this result. Costinot et al. (2014) show that countries have incentives to manipulate their terms of trade through taxes on capital flows only when their share of world output fluctuates over time. In our model, if countries could freely issue equity, then their share of world output would be constant because of (perfect) risk sharing; with complete markets, there would be no reason to manipulate the terms of trade. We numerically solve for coordinated policies chosen by a global social planner and for uncoordinated policies chosen in Nash equilibrium and find that, due to these strategic incentives, Nash policies 
are tighter than would be chosen by a social planner. A global social planner would (for most Pareto weights) choose some degree of financial openness in the AE, allowing limited leverage and credit inflows to provide better international risk sharing because the $\mathrm{AE}$ has a higher risk tolerance. However, in Nash equilibrium the macro-level spillovers and strategic incentives lead both countries to close credit flows completely (maximally regulate).

Crucially, the differences between Nash and optimal coordinated policies have asymmetric impacts on the AE and EME. When the AE does not use prudential controls but allows credit inflows and domestic leverage, the more risk averse EME benefits from better international risk sharing since the AE acts as a global intermediary, borrowing with safe debt to invest in risky capital. In Nash equilibrium, however, the AE is unwilling to act as a global intermediary, recognizing that credit flows create financial instability (longer and more frequent crises) that mostly harms its own agents to the benefit of the EME. In other words, because markets are incomplete, the so-called “exorbitant privilege" (excess return) from intermediating global risk does not sufficiently compensate the so-called "exorbitant duty" (losses in crises) of being the primary bearer of financial instability. Relative to the laissez-faire competitive equilibrium and to coordinated outcomes that place less than $80 \%$ Pareto weight on the AE, the AE prefers the Nash equilibrium because it mitigates exposure to long-run financial instability. In contrast, the EME's welfare outcomes are worse under Nash policies. In principle, policy coordination leads to outcomes at least as good as the uncoordinated equilibrium, but achieving successful coordination is nontrivial, and the benefits may accrue unequally to participants. Understanding the incentives for coordination and the incentives that cause Nash equilibrium to differ from the coordinated equilibrium offer important insight for the conduct and creation of new international regulations.

Related Literature. Our paper follows the stochastic continuous-time macro literature, pioneered by Brunnermeier and Sannikov (2014a, 2015, 2016a) and He and Krishnamurthy (2012, 2013, 2014), who apply continuous-time methods to analyze the non-linear global dynamics of economies with financial frictions. Most closely related, Brunnermeier and Sannikov (2015) demonstrate that closed capital accounts can improve welfare relative to open accounts because closing capital accounts reduce global instability arising from incomplete markets. We extend the 
analysis of Brunnermeier and Sannikov (2015) in two main ways: first, we allow each country to choose occasionally binding limits on capital inflows, with countries choosing potentially different policies; second, we use recursive preferences to disentangle effects driven by risk aversion from intertemporal substitution. By giving countries more flexibility in their policy choices, our model admits interesting policy spillovers and strategic considerations.

Our paper connects the literatures on the impact of capital controls on terms of trade and on macroprudential policy coordination (see below). In Costinot et al. (2014), for example, countries optimally "manipulate" the terms of trade by differentially taxing capital inflows (outflows) when growth is higher (lower) than the rest of the world. We show that manipulating the terms of trade with simple leverage constraints on capital outflows proves an effective macroprudential tool in an economy with long-run financial instability due to incomplete markets. As in Costinot et al. (2014), the terms of trade creates individual incentives that are not necessarily aligned with social incentives. The literature on macroprudential policy coordination has studied several cases in which countries' policy choices create spillovers. Our novel insight and main contribution is that in a financially asymmetric world, this terms-of-trade mechanism has different normative implications for the welfare of AEs and EMEs. Incentives to manipulate the terms of trade complicates macroprudential policy coordination between AEs and EMEs because it misaligns their objectives. Both countries want to mitigate exposure to systemic risk, but EMEs want AEs to provide intermediation, which requires bearing some systemic risk.

Economists have tended to focus on the use of capital controls by EMEs to induce favorable terms of trade (see e.g. Costinot et al. 2014; Farhi and Werning, 2014) or to mitigate the adverse consequences of fickle capital flows (see e.g. Caballero and Simsek, 2016). Similar to Costinot et al. (2014), Farhi and Werning (2014), and Heathcote and Perri (2016), in our model capital controls work by changing a country's terms of trade. However, the mechanism in our model is quite different. Rather than manipulating the risk-free interest rate and consumption behavior, capital controls primarily affect the terms of trade hedge identified by Brunnermeier and Sannikov (2015), which has a meaningful impact because of incomplete international markets. Limiting inflows alone is sufficient to benefit a country because the terms of trade hedge changes the sta- 
tionary distribution of an economy, dynamically benefiting the financial position of the country in the long-run. An advantage of this approach is the simplicity of the policy instrument, as opposed to dynamically adjusting taxes on capital inflows/outflows.

The literature on macroprudential policy and pecuniary externalities includes Caballero and Krishnamurthy (2001, 2004), Aoki et al. (2009, 2010), Bianchi (2010, 2011), Mendoza (2010), and Bianchi and Mendoza (2010). Korinek (2016) provides a "first welfare theorem" when international markets lack frictions; there is role for coordination in our setting because international financial markets are imperfect. Within the continuous-time literature, Caballero and Simsek (2017) consider how macroprudential policy can mitigate damage arising from belief heterogeneity, and Phelan (2016) illustrates that leverage limits improve macroeconomic stability by endogenously increasing the frequency with which the banking sector is well-capitalized. These results contrast with Gârleanu et al. (2015), where limits on leverage reduce agents' incentives to diversify and result in more correlated investments, increasing financial fragility.

Our model contributes to the literature on coordinated policy in international settings due to spillovers. Banerjee et al. (2016) explore the impact of spillovers from the macroeconomic policies of advanced economies to emerging market economies. Acharya and Bengui (2018) study capital flow management when AEs are at the ZLB. Jin and Shen (2019) studies macroprudential policy coordination spillovers among EMEs, who over-accumulate liquid assets, thus pushing down global rates. Sergeyev (2014) studies policy coordination in response to bank funding shocks, and global coordination works through affecting interest rate.

Similar to our analysis, Agénor et al. (2018) consider a linearized core-periphery model when financial intermediation is subject to frictions and show that policies trade off mitigating the shortrun consequences of the financial accelerator (and spillovers) and the long-run effects of higher costs of capital. Our paper is closely related to Davis and Devereux (2019), who analyze an asymmetric two-country economy where one country faces domestic financial frictions and the other does not. Our result that tighter policy improves stability is consistent with the evidence in Boar et al. (2017) who find that countries that more frequently use macroprudential tools, other things being equal, experience stronger and less volatile GDP growth. Our result that tighter 
policy provides strategic incentives for another country to tighten is consistent with the evidence in Agénor et al. (2018) who find that countries tend to set macroprudential policy more actively if their "neighbors" are more active.

Outline. The remainder of the paper is organized as follows. Section 2 presents the model and the equilibrium conditions. Section 3 numerically solves for equilibrium in a calibrated economy and illustrates the effects of regulations. Section 4 solves for optimal coordinated and uncoordinated policies (constant/fixed leverage limits). Section 5 concludes.

\section{The Model}

This section presents a modified version of the two-country model in Brunnermeier and Sannikov (2015). The global economy is populated by agents who live in two different countries, the AE and the EME. Variables related to the AE and the EME will be denoted by a subscript of $A$ and $E$, respectively. Agents use capital to produce the two intermediate consumption goods $a$ and $b$, and agents in the $\mathrm{AE}$ have productive advantages at producing good $a$ (vice versa the EME and b). Capital trades in a competitive market, but agents in each country may be subject to leverage constraints owing to capital account policies in each country. Financial frictions limit international credit flows to risk-free debt (non-state contingent contracts).

\subsection{Setup}

Technology. Time is infinite and continuous. Capital can be used to produce either good $a$ or good $b$, which are then combined into an aggregate (final) consumption good $y_{t}$, given by

$$
y_{t}=\left(y_{a, t}\right)^{\alpha}\left(y_{b, t}\right)^{1-\alpha},
$$

where $y_{a, t}$ is the supply of good and $y_{b, t}$ is the supply of good $b$.

Agents can produce goods $a$ and $b$ using linear production technologies, but agents in the AE have a superior technology for good $a$ while agents in the EME have a superior technology for 
good $b$. Given $k_{t}$ units of a capital, agents in the AE produce good $a$ at rate $\bar{a} k_{t}$ and good $b$ at rate $\underline{a} k_{t}$, where $\bar{a}>\underline{a}>0$. The EME agents face the reverse situation, with good $a$ produced at rate $\underline{a} k_{t}$ and good $b$ at rate $\bar{a} k_{t}$. Because of these productivity differences, output endogenously depends on agents' risk-taking decisions $3^{3}$

Denote the aggregate amount of world capital available at time $t$ by $K_{t}$, and denote the share of world capital held by agents in the AE and in the EME by $\psi_{A, t}$ and $\psi_{E, t}$, respectively. Denote the fraction of world capital devoted to production of goods $a$ and $b$ by $\psi_{A a, t}, \psi_{A b, t}, \psi_{E a, t}$, and $\psi_{E b, t}$, where the first superscript denotes the country employing the capital. By definition,

$$
\psi_{A a, t}+\psi_{A b, t}+\psi_{E a, t}+\psi_{E b, t}=1
$$

The supply of goods $a$ and $b$ per unit of capital then are given by

$$
Y_{a, t}=\bar{a} \psi_{A a, t}+\underline{a} \psi_{E a, t}, \quad Y_{b, t}=\underline{a} \psi_{A b, t}+\bar{a} \psi_{E b, t},
$$

yielding the total supply of the aggregate good

$$
Y_{t} K_{t}=\left(Y_{a, t}\right)^{\alpha}\left(Y_{b, t}\right)^{1-\alpha} K_{t}
$$

Let the final good be the numeraire. Then the prices of $a$ and $b$ can be written as

$$
P_{a, t}=\alpha\left(\frac{Y_{b, t}}{Y_{a, t}}\right)^{1-\alpha}, \quad P_{b, t}=(1-\alpha)\left(\frac{Y_{a, t}}{Y_{b, t}}\right)^{\alpha} .
$$

\footnotetext{
${ }^{3}$ Our main insights would still hold if output followed an endowment process and countries traded claims to endowments because the incentive to manipulate terms of trade would remain (see Costinot et al. (2014)). However, we prefer a model with endogenous output for two reasons. First, a stationary distribution generally will not exist unless multiple trees and asymmetric consumption baskets are introduced, at which point the consequent model would be more complicated, not less. For intuition, suppose final output followed a single endowment process. The model would be the same as the case of no productivity differences in Brunnermeier and Sannikov (2015), who show that the stationary distribution would be degenerate. The issue is that there are no forces in the model that cause mean reversion in the share of global wealth. Multiple endowment processes with a symmetric consumption basket would not change this problem. Second, in the real world, countries do face incomplete international markets and have to manage a fundamental trade off between static efficiency of capital allocation and dynamic stability. We want to better understand how countries behave when financial crises have consequences for real economic activity.
} 
There is a single type of physical capital. We model productivity shocks as shocks directly to capital, which can be interpreted as shocks to "effective capital." Capital owned by agents in Country $i$ evolves according to

$$
\frac{d k_{t}}{k_{t}}=\left(\Phi\left(l_{t}\right)-\delta\right) d t+\sigma d W_{i, t}
$$

where $\Phi(\cdot)$ is a standard investment technology: ${ }_{4}^{4}>0$ is the rate of depreciation, $\sigma$ is the volatility of capital growth, and $W_{i, t}$ is a standard Brownian motion. The two Brownian motions, $W_{A, t}$ and $W_{E, t}$, are independent and exogenous.

This specification is tractable and admits the interpretation of global aggregate shocks with idiosyncratic (negatively correlated) country specific shocks. Thus, the shocks can capture countryspecific productivity gains as well as redistributive shocks (e.g. international law suits). The reader may interpret these shocks as either shocks to capital located in a specific country (as in Brunnermeier and Sannikov (2015) ) or productivity shocks based on the owner's identity 5 Natural disasters are an example of the former while changes in a country's technical knowledge are an example of the latter. One advantage of the latter interpretation is that physical capital does not need to cross borders when reallocated between countries. Thus, an agent in country $i$ buys capital, wherever it is physically located, but the productivity of capital, and shocks to productivity, are determined by the owner's country of residence. ${ }^{6}$ The reader should choose whichever interpretation they prefer. As long as the shocks are either only to capital physically located in country $i$ or only to capital owned by country $i$, the model is mathematically identical. 7 It follows that aggregate

\footnotetext{
${ }^{4}$ In previous versions of this paper, capital grew according to an exogenous rate rather than with adjustment costs. The results are qualitatively and quantitatively similar. We choose this set up to stay closer to the modeling choices in Brunnermeier and Sannikov (2015).

${ }^{5}$ In reality shocks may depend on both the country location and the country of ownership. For tractability in our paper we assume that only one of these matters. Appendix F shows that risk premia in our baseline model are similar to risk premia in a model which includes shocks dependent on both country location and the owner's country of residence. In the case of unit elasticity of substitution and risk aversion, there exists parametrizations such that risk premia are the same.

${ }^{6}$ This assumption implies that if Apple runs a factory in China, the productivity of the factory is determined by Apple, and changes in productivity are driven by changes in Apple's characteristics.

${ }^{7}$ The specification of shocks in our model is mathematically identical to the specification in Brunnermeier and Sannikov (2015). We mention this point to address concerns we have previously received that shocks based on ownership are nontrivially different from location-specific shocks. The interpretation of the shocks matters for a quantitative estimation, but our purposes are theoretical. The actual equations are not changed, only the mapping to data.
} 
capital follows the law of motion

$$
\frac{d K_{t}}{K_{t}}=\left(\Phi\left(\boldsymbol{l}_{t}\right)-\delta\right) d t+\psi_{A, t} \sigma d W_{A, t}+\psi_{E, t} \sigma d W_{E, t}
$$

To economize on notation, we will write $\mu_{K, t}=\Phi\left(\boldsymbol{l}_{t}\right)-\delta, \Sigma_{K, t}=\left[\psi_{A, t} \sigma, \psi_{E, t} \sigma\right]^{T}$, and $d \mathbf{W}_{t}=$ $\left[d W_{A, t}, d W_{E, t}\right]^{T}$.

Preferences. Agents have recursive preferences as in Duffie and Epstein (1992). Let $\gamma_{i}$ be the risk aversion of agents in Country $i$. Agents in the AE have lower risk aversion $\left(\gamma_{A} \leq \gamma_{E}\right)$, which implies that in equilibrium the AE will generally act as the "global intermediary" (use leverage). 8 To focus our results on the role of the $\mathrm{AE}$ as the global intermediary, we assume a common discount rate $\rho$ and unit elasticity of intertemporal substitution (EIS) $\varphi^{9}$ The lifetime utility $V_{i, 0}$ of Country $i$ agents is given by

$$
V_{i, 0}=\mathbb{E}\left[\int_{0}^{\infty} f^{i}\left(c_{i, t}, V_{i, t}\right) d t\right]
$$

where $f^{i}(\cdot)$ is the aggregator

$$
f^{i}\left(c_{i, t}, V_{i, t}\right)=\rho\left(1-\gamma_{i}\right) V_{i, t}\left(\log \left(c_{i, t}\right)-\frac{1}{1-\gamma_{i}} \log \left(\left(1-\gamma_{i}\right) V_{i, t}\right)\right)
$$

Markets for Physical Capital and Risk-free Bonds. Agents can trade ownership of physical capital in a competitive international market. We denote the equilibrium market price of capital per unit by $q_{t}$. Hence, capital $k_{t}$ has market value $q_{t} k_{t}$. We postulate that $q_{t}$ evolves endogenously

\footnotetext{
${ }^{8}$ Asymmetry in the volatility of capital growth $\left(\sigma_{A}<\sigma_{E}\right)$ is an alternative modeling approach that we have used in previous versions of this paper. This approach delivers the same qualitative results regarding global risk bearing. Intuitively, a lower $\sigma_{A}$ causes agents in the AE to price capital as if their risk aversion was lower than the EME's. See Footnote 13 for the exact equation. We prefer asymmetric risk aversion, however, because it focuses on the AE's role as a global intermediary rather than a particular micro-foundation for why the AE has a superior risk-bearing capacity.

${ }^{9}$ By using recursive preferences rather than CRRA preferences, we know that our results are driven by asymmetry in risk aversion rather than asymmetry in EIS. Our results also hold under CRRA preferences since the central mechanism is the connection between financial asymmetry and the terms of trade for intermediate goods, not the incentive to consume and save intertemporally.
} 
according to

$$
\frac{d q_{t}}{q_{t}}=\mu_{q, t} d t+\sigma_{q A, t} d W_{A, t}+\sigma_{q E, t} d W_{E, t}
$$

where $\mu_{q, t}, \sigma_{q A, t}$, and $\sigma_{q E, t}$ will be determined in equilibrium. The capital price determines the rate of internal investment according to Tobin's q:

$$
\Phi^{\prime}\left(\boldsymbol{l}_{t}\right)=\frac{1}{q_{t}}
$$

Agents can also trade a risk-free bond that is in zero net supply. We denote the endogenously determined risk-free return by $d r_{t}^{F}$ (we implicitly assume that the risk-free interest rate has absolutely continuous paths). Agents can borrow or save in the risk-free asset, but they may face borrowing limits imposed by regulation in their country. Agents cannot sell short capital.

Returns from Capital. The return from holding capital can be written as diffusion processes by summing capital gains $d\left(q_{t} k_{t}\right) /\left(q_{t} k_{t}\right)$, which has two volatility terms, and the dividend yield from using capital to produce good $a$ or $b$, which has no volatility terms. We denote the return from an agent in Country $i$ buying physical capital and using it to produce good $j$ by $d r_{t}^{i j}$. Given equations (4) and (8) for the laws of motion for capital and the capital price and using Ito's Lemma, returns for the AE when producing good $a$ are given by

$$
d r_{t}^{A a}=\left(\frac{\bar{a} P_{a, t}}{q_{t}}+\mu_{q, t}+\Phi\left(l_{t}\right)-\delta+\sigma \sigma_{q A, t}\right) d t+\left(\sigma+\sigma_{q A, t}\right) d W_{A, t}+\sigma_{q E, t} d W_{E, t}
$$

and analogously for the other country-goods pairs ${ }^{10}$ Notably, the AE shocks affect the returns to capital used in the AE directly ( $k_{t}$ changes) and indirectly through the effect on the capital price $q_{t}$; however, the EME's shocks affect returns indirectly through the effect on the capital price (analogously for how shocks affect the EME's returns). To economize on notation, we will let $\Sigma_{i j, t}$ denote the volatility vector for Country $i$ when producing good $j$. For example, the volatility vector of investing in good $a$ for the AE is $\Sigma_{A a, t}=\left[\sigma+\sigma_{q A, t}, \sigma_{q E, t}\right]^{T}$. Note that $\Sigma_{i a, t}=\Sigma_{i b, t}$.

$$
{ }^{10} \text { e.g., } d r_{t}^{E b}=\left(\frac{\bar{a} P_{b, t}}{q_{t}}+\mu_{q, t}+\Phi\left(l_{t}\right)-\delta+\sigma \sigma_{q E, t}\right) d t+\sigma_{q A, t} d W_{A, t}+\left(\sigma+\sigma_{q E, t}\right) d W_{E, t} .
$$


Incomplete Markets, Financial Frictions, and Prudential Regulations. The key financial friction in this model is the inability of countries to issue equity to each other. Agents can only trade risk-free bonds to purchase capital ownership, and as a result, markets are incomplete because they are limited to non-contingent financial contracts and cannot sell short capital. This reliance on riskfree debt generates financial instability that would be attenuated if agents could purchase equity in the other country. If markets were complete, then countries would not apply prudential regulations or capital controls. With free issuance of equity, countries could perfectly share aggregate risk and consume constant shares of world output. Costinot et al. (2014) shows that countries only have an incentive to manipulate capital flows only when their share of world output fluctuates over time.11 In addition, agents cannot sell short investment in the production of goods $a$ and $b$. Incomplete international markets can be motivated by home bias in equity holdings and micro-founded by agency problems and asymmetric information. 12

The heart of our analysis involves prudential regulations in each country. We model prudential regulations as borrowing limits requiring that leverage from capital inflows not exceed a countryspecific threshold. Within the context of our model, leverage limits have the effect of limiting aggregate borrowing and therefore aggregate inflows. Maintaining the interpretation that capital ownership could be across borders, leverage limits would therefore apply to total balance sheets (holdings of domestic and foreign capital assets), and so limits on borrowing via debt will limit total asset holdings. In reality, macroprudential policies such as leverage limits are typically designed to target domestic excesses, not to address capital flow imbalances, and capital controls are envisioned as separate sets of tools. But the distinction is not always so clear. One example

\footnotetext{
${ }^{11}$ Consider the example in Section IIIC of Costinot et al. (2014). When there are aggregate fluctuations, countries have incentives to tax capital inflows when they expect to earn a larger share of world output tomorrow and tax capital outflows in the opposite case. If they expect to earn the same share of world output tomorrow, however, then they have no incentive to manipulate the terms of trade. This example is the proper mapping of their paper to our model, where output is effectively exogenous under perfect risk sharing but becomes endogenous with incomplete markets.

${ }^{12}$ Heathcote and Perri (2013) provide an explanation for the empirically observed bias toward domestic asset holdings. See Jensen and Meckling (1976), Bolton and Scharfstein (1990), and Holmström and Tirole (1997) for theories of agency problems and asymmetric information limiting state-contingent contracts. More broadly, a home equity bias can be rooted in a fundamental trade-off between the diversification benefits and participation costs of investing in international markets. Gârleanu et al. (2015) show that limited market integration endogenously arises because of this trade-off, and since portfolios comprise more correlated securities, risk premia are higher relative to the frictionless benchmark.
} 
in which macroprudential policies were used to affect capital inflows is the case of Peru, which limited banks' derivatives positions as a way of stemming capital flows (see Keller, 2018). If the reader prefers a single interpretation of prudential limits in our model, then limits on capital inflows is the more robust interpretation, since heterogeneity within each country would imply that domestic leverage limits could have different effects from limits on aggregate inflows.

Consumption and Portfolio Choice. Each agent in Country $i$ decides her consumption rate $c_{i, t}$ as well as how to allocate remaining wealth. Agents face a portfolio choice problem of how much capital to invest in the production of goods $a$ and $b$, and how much to invest in the risk-free bond. For Country $i$, we denote the portfolio weights by $\left(x_{i a, t}, x_{i b, t}, 1-x_{i a, t}-x_{i b, t}\right)$, where $x_{i a, t}$ is the fraction of wealth invested in capital used to produce good $a, x_{i b, t}$ is the fraction of wealth invested in capital used to produce $\operatorname{good} b$, and $1-x_{i a, t}-x_{i b, t}$ is the fraction of wealth invested in the risk-free asset. Portfolio weights $x_{i a, t}$ and $x_{i b, t}$ must be non-negative.

Denote $L_{A, t}$ and $L_{E, t}$ as the leverage constraints imposed on the AE and EME, respectively (we restrict leverage constraints to be deterministic functions of the state variable). These constraints impose that the market value of an agent's borrowing (debt) cannot exceed $L_{i, t}$ times their wealth (leverage of 0 implies no borrowing). In other words, for an agent in Country $i$

$$
x_{i a, t}+x_{i b, t} \leq L_{i, t}+1 \text {. }
$$

Given a consumption rate $c_{i, t}$ and portfolio weights $\left(x_{i a, t}, x_{i b, t}, 1-x_{i a, t}-x_{i b, t}\right)$, the the net worth $n_{i, t}$ of an agent evolves according to

$$
\frac{d n_{i, t}}{n_{i, t}}=x_{i a, t} d r_{t}^{i a}+x_{i b, t} d r_{t}^{i b}+\left(1-x_{i a, t}-x_{i b, t}\right) d r_{t}^{F}-\frac{c_{i, t}}{n_{i, t}} d t
$$

Thus, agents' problems can be summarized as maximizing utility (6) subject to the budget constraint (12) together with the solvency constraint $n_{t} \geq 0$ and the borrowing constraint (11). With unit EIS, agents optimally consume a fraction $\rho$ of net wealth, implying $c_{t}=\rho n_{t}$ for all agents.

Definition 1 (Equilibrium). For any initial allocation of wealth, an equilibrium is a map from 
histories of shocks $\left\{W_{s}^{A}, W_{s}^{E}, s \in[0, t]\right\}$ to the allocation of capital $\left(\psi_{A a, t}, \psi_{A b, t}, \psi_{E a, t}, \psi_{E b, t}\right)$ and the aggregate consumption good $\left(C_{A, t}, C_{E, t}\right)$ as well as price $q_{t}$ and risk-free rate $d r_{t}^{F}$ such that

1. All agents solve their optimal consumption and portfolio choice problems, subject to the solvency constraint on their net worth and leverage constraints.

\section{Capital, consumption, and debt markets clear.}

\subsection{Solving for Equilibrium}

Since countries cannot issue equity internationally, an agent's portfolio decisions depend on her level of wealth, so equilibrium depends on the aggregate level of wealth in each country. As the AE's wealth increases, its aggregate capital holdings will increase, which will increase the fraction of global capital used to produce good $a$. Thus, capital allocations and final good production will depend on country's relative wealth, which will vary in response to global shocks. We use stochastic continuous-time methods to solve for global equilibrium dynamics. We solve for a recursive (or Markov), rational-expectations equilibrium in which the single state variable is the relative wealth of the two countries.

Denote the aggregate net worth of agents in the AE at time $t$ by $N_{A, t}$. Then the relative share of

net wealth held by the AE is defined to be $\eta_{t} \equiv \frac{N_{A, t}}{q_{t} K_{t}}$. Thus, $\eta_{t}$ represents the share of global wealth held by the AE. The aggregate portfolio choice of the AE and EME can be written respectively as

$$
\left(\frac{\psi_{A a, t}}{\eta_{t}}, \frac{\psi_{A b, t}}{\eta_{t}}, 1-\frac{\psi_{A, t}}{\eta_{t}}\right), \quad\left(\frac{\psi_{E a, t}}{1-\eta_{t}}, \frac{\psi_{E b, t}}{1-\eta_{t}}, 1-\frac{\psi_{E, t}}{1-\eta_{t}}\right)
$$

where $\psi_{A, t}=\psi_{A a, t}+\psi_{A b, t}$ and $\psi_{E, t}=\psi_{E a, t}+\psi_{E b, t}$. Equilibrium consists of an endogenous law of motion for $\eta_{t}$ and capital allocations and prices that are functions of the state variable $\eta_{t}$.

The Value Function. The aggregator function (7) implies agents in the AE and the EME have the value functions

$$
V_{A, t}=\frac{\left(\xi_{t} n_{A, t}\right)^{1-\gamma_{A}}}{1-\gamma_{A}}, \quad V_{E, t}=\frac{\left(\zeta_{t} n_{E, t}\right)^{1-\gamma_{E}}}{1-\gamma_{E}}
$$


where $\xi_{t}$ and $\zeta_{t}$ capture the forward-looking value of an additional dollar of net worth. We postulate that in equilibrium

$$
\begin{gathered}
\frac{d \xi_{t}}{\xi_{t}}=\mu_{\xi, t} d t+\Sigma_{\xi, t} \cdot d \mathbf{W}_{t}, \\
\frac{d \zeta_{t}}{\zeta_{t}}=\mu_{\zeta, t} d t+\Sigma_{\zeta, t} \cdot d \mathbf{W}_{t} .
\end{gathered}
$$

Asset Pricing Equations. When leverage constraints do not bind, agents choose portfolios such that excess returns equal the covariance of the returns with their stochastic discount factor (SDF). We can separate the covariance into two terms. For AE agents, the first term is denoted by $\varsigma_{A a, t}$, and the second term is denoted by $\varsigma_{\xi A, t}$. The term $\varsigma_{A a, t}$ captures the "fundamental" variance of returns from holding capital whereas the term $\varsigma_{\xi A, t}$ captures the covariance between the marginal value of AE agents' net worth $\xi_{t}$ and returns from holding capital. We can analogously define $\zeta_{E b, t}$ and $\zeta_{\zeta E, t}$. However, when leverage constraints bind, the excess returns can exceed the risk premium. We prove these claims by examining agents' Hamilton-Jacobi-Bellman (HJB) equations. For details, see Appendix A.

The leverage constraint will bind for agents in the $\mathrm{AE}$ when, if they were subject to no constraints, $\psi_{A, t} / \eta_{t} \geq L_{A, t}+1$ and in the EME when, if they were subject to no constraints, $\psi_{E, t} /(1-$ $\left.\eta_{t}\right) \geq L_{E, t}+1$. Thus $\psi_{A, t} / \eta_{t} \leq L_{A, t}+1$ and $\psi_{E, t} /\left(1-\eta_{t}\right) \leq L_{E, t}+1$ over the state space.

Characterizing Equilibrium. Using the returns equations and our conjectures on agents' value functions, together with market clearing for capital and consumption, we can characterize equilibrium as a system of differential equations in the capital price $q_{t}$, the AE's value of additional net worth $\xi_{t}$, and the EME's value of additional net worth $\zeta_{t}{ }_{13}^{13}$ This system depends on the endogenous state variable $\eta_{t}$. Here, we report the law of motion for $\eta_{t}$ and characterize the state space. We derive the law of motion and the system of differential equations in Appendix A.

\footnotetext{
${ }^{13}$ The AE's and EME's HJB equations give ODEs for $\xi_{t}$ and $\zeta_{t}$. The ODE for $q_{t}$ is obtained by differencing the AE's and EME's asset pricing equations. For example, if both countries specialize their production, then

$$
\begin{aligned}
\mathbb{E}\left[d r_{t}^{A a}\right]-\mathbb{E}\left[d r_{t}^{E b}\right] & =\gamma_{A} \varsigma_{A a, t}+\left(\gamma_{A}-1\right) \varsigma_{\xi A, t}-\gamma_{E} \varsigma_{E b, t}-\left(\gamma_{E}-1\right) \varsigma_{\zeta E, t} \\
& =\gamma_{A} \frac{\psi_{A, t}}{\eta_{t}}\left(\left(\sigma+\sigma_{q A, t}\right)^{2}+\sigma_{q E, t}^{2}\right)+\left(\gamma_{A}-1\right) \varsigma_{\xi A, t}-\gamma_{E} \frac{\psi_{E, t}}{1-\eta_{t}}\left(\sigma_{q A, t}^{2}+\left(\sigma+\sigma_{q E, t}\right)^{2}\right)-\left(\gamma_{E}-1\right) \varsigma_{\zeta E, t}
\end{aligned}
$$
}


First, we characterize equilibrium when leverage constraints do not bind.

Proposition 1. When leverage constraints do not bind, the equilibrium law of motion of $\eta$ will be endogenously given as

$$
\frac{d \eta_{t}}{\eta_{t}}=\mu_{\eta, t} d t+\sigma_{\eta A, t} d W_{A, t}+\sigma_{\eta E, t} d W_{E, t}
$$

where

$$
\begin{aligned}
\mu_{\eta, t}= & \frac{\bar{a} P_{a, t}-\imath_{t}}{q_{t}}-\rho+\left(\frac{\psi_{A, t}}{\eta_{t}}-1\right)\left(\gamma_{A} \varsigma_{A a, t}+\left(\gamma_{A}-1\right) \varsigma_{\xi A, t}\right) \\
& -\left(\frac{\psi_{A, t}}{\eta_{t}} \Sigma_{A a, t}-\left(\Sigma_{q, t}+\Sigma_{K, t}\right)\right) \cdot\left(\Sigma_{q, t}+\Sigma_{K, t}\right)-\sigma \sigma_{q E, t} \\
\sigma_{\eta A, t}= & \frac{1-\eta_{t}}{\eta_{t}} \psi_{A, t} \sigma+\left(\frac{\psi_{A, t}}{\eta_{t}}-1\right) \sigma_{q A, t} \\
\sigma_{\eta E, t}= & -\psi_{E, t} \sigma+\left(\frac{\psi_{A, t}}{\eta_{t}}-1\right) \sigma_{q E, t} .
\end{aligned}
$$

The state space is divided into 3 regions. For $\eta<\eta^{a}$, both countries produce good a and the EME produces good $b$. For $\eta>\eta^{b}$ both countries produce good $b$ and the AE produces good $a$. For $\eta \in\left[\eta^{a}, \eta^{b}\right]$ countries specialize, using only their most productive technology. Goods prices satisfy

$$
\frac{\underline{a}}{\bar{a}} \leq \frac{P_{b, t}}{P_{a, t}} \leq \frac{\bar{a}}{\underline{a}}
$$

where the first (second) inequality becomes equality in the left (right) region of the state space.

Equilibrium is slightly modified when leverage constraints bind. In this case, the constraint prevents agents from taking as much risk as they demand. As a result, their excess returns will not equal the covariance of their returns with their SDF. We deal with this issue by observing that in equilibrium either the AE or the EME is a net debtor. Suppose, for concreteness, that leverage constraints bind for the AE. Then the EME must be a net lender, so its agents cannot be

A lower $\gamma_{A}$ causes RHS to shrink. To maintain equality, all else equal, the AE takes more leverage and increases $\psi_{A, t}$. This equation is similar in the case of asymmetric $\sigma$. For simplicity, let $\gamma_{A}=\gamma_{E}=1$. Then

$$
\mathbb{E}\left[d r_{t}^{A a}\right]-\mathbb{E}\left[d r_{t}^{E b}\right]=\frac{\psi_{A, t}}{\eta_{t}}\left(\left(\sigma_{A}+\sigma_{q A, t}\right)^{2}+\sigma_{q E, t}^{2}\right)-\frac{\psi_{E, t}}{1-\eta_{t}}\left(\sigma_{q A, t}^{2}+\left(\sigma_{E}+\sigma_{q E, t}\right)^{2}\right) .
$$

A lower $\sigma_{A}$ similarly decreases the RHS, so the AE increases $\psi_{A, t}$, all else equal. 
constrained, hence their excess returns still equal the covariance of their returns with their SDF. We use this observation to re-express $d \eta_{t}$. The state variable $\eta_{t}$ will follow this new law of motion until the leverage constraint no longer binds, at which point the law of motion reverts to (16).14

Proposition 2. If the EME's leverage constraint binds, then $\eta_{t}$ continues to follow the law of motion in Proposition 1. When the AE's leverage constraint binds, $\psi_{A, t}=\left(1+L_{A, t}\right) \eta_{t}$, and the drift of $\eta_{t}$ becomes

$$
\begin{aligned}
\mu_{\eta t}= & \left(\frac{\psi_{A, t}}{\eta_{t}}-1\right)\left(\gamma_{E} \varsigma_{E b, t}+\left(\gamma_{E}-1\right) \varsigma_{\zeta E, t}-\frac{\bar{a} P_{b, t}}{q_{t}}\right)+\frac{1}{\eta_{t} q_{t}}\left(\psi_{A a, t} \bar{a} P_{a, t}+\psi_{A b, t} \underline{a}_{b, t}\right) \\
& -\frac{l_{t}}{q_{t}}-\rho+\left(\frac{\psi_{A, t}}{\eta_{t}}-\psi_{A, t}-1\right) \sigma\left(\sigma_{q A, t}-\sigma_{q E, t}\right) \\
& -\left(\frac{\psi_{A, t}}{\eta_{t}} \Sigma_{A a, t}-\left(\Sigma_{q, t}+\Sigma_{K, t}\right)\right) \cdot\left(\Sigma_{q, t}+\Sigma_{K, t}\right) .
\end{aligned}
$$

The volatility vector $\Sigma_{\eta, t}$ and the division of the state space remain the same as in (16).

\section{Numerical Results}

We numerically solve our model using the method of the false transient. For details, we refer the reader to Appendix C. For the remainder of this paper, we will suppress time subscripts.

\subsection{Calibration}

Table 1 lists the parameter values we use. While our goal is not a quantitative model, we calibrate our model parameters to be reasonable. We set the risk aversions of the AE and EME so that they are not far from 1 and 2 , respectively. The discount rate $\rho$ is set at $4 \%$ following the continuous-time macrofinance literature. We normalize $\bar{a}=1$ and let $\underline{a}=0.85$ to reflect gains from specialization in trade of $18 \%$, but we also check our results are robust to other values of $\underline{a}$ in

\footnotetext{
${ }^{14}$ Even though $\eta_{t}$ follows the same law of motion when leverage constraints do not bind, it does not necessarily imply that, as functions of $\eta$, the equilibrium prices, volatilities, and capital allocations are the same in regions of the state space where constraints do not bind. For example, if $L_{A, t}$ does not bind when $\eta_{t}>0.3$, it does not imply that, for $\eta_{t}>0.3, \psi_{A a, t}$ in the equilibrium with an occasionally binding leverage constraint agrees everywhere with $\psi_{A a, t}$ in the equilibrium with no leverage constraints.
} 
Table 1: This table lists the model's parameters, our chosen values, the quantities to match, the target numbers, and the model's calculations. If the quantities to match are taken from the literature or chosen by another rationale, we do not report the target and the model's calculation.

\begin{tabular}{lccllc}
\hline \hline \multirow{2}{*}{ Description } & & & & \\
& Parameter & Value & Quantity to Match & Target & Model \\
\hline \hline & & & & & \\
Risk aversion of the AE & $\gamma_{A}$ & 1.05 & - & - & - \\
Risk aversion of the EME & $\gamma_{E}$ & 2 & - & - & - \\
Discount rate & $\rho$ & $4 \%$ & Literature & - & - \\
First-Best Productivity & $\bar{a}$ & 1 & Normalized & - & - \\
Second-Best Productivity & $\underline{\lambda}$ & 0.85 & Specialization gains & - & - \\
Investment Tech Productivity & $\lambda$ & 57 & Consumption growth & $2 \%$ & $1.9 \%$ \\
Investment Tech Illiquidity & $\kappa$ & 5200 & Investment/output ratio & $20 \%$ & $21 \%$ \\
Depreciation rate & $\delta$ & $5 \%$ & Literature & - & - \\
Capital Growth Volatility & $\sigma$ & $2 \%$ & Consumption volatility & $2 \%$ & $2 \%$ \\
Cobb-Douglas Weight & $\alpha$ & $1 / 2$ & Symmetry & - & - \\
& & & & & \\
\hline \hline
\end{tabular}

Appendix E For computational tractability, we choose the following functional form for $\Phi(\cdot)$ :

$$
\Phi(\imath)=\frac{\lambda}{\kappa} \log (\kappa \imath+1) .
$$

We calibrate the parameters $\lambda$ and $\kappa$ to match a $2 \%$ growth rate of aggregate U.S. consumption and an investment to output ratio of $20 \%$, following Di Tella (2017). The depreciation rate $\delta$ is set at 5\% following Brunnermeier and Sannikov (2015) and Di Tella (2017). Finally, we set $\alpha=1 / 2$ so that overall demand for intermediate goods $a$ or $b$ does not influence our results, following Brunnermeier and Sannikov (2015).

For simplicity, we suppose countries choose a fixed, state-independent leverage constraint $L_{A}$ and $L_{E}$. We first consider how varying constraints affect the stationary distribution (stability) of the global economy and then consider how constraints in each country affect welfare in the AE. Our results do not depend on asymmetry-the effects of leverage constraints on each country are qualitatively similar if $\gamma_{A}=\gamma_{E}$, and each country has an incentive to "over-regulate" in Nash 
equilibrium - but the distributional consequences depend on asymmetric risk tolerance. Note that the numerical results in Brunnermeier and Sannikov (2015) correspond to a special case of our model with $\gamma_{A}=\gamma_{E}=1$ (the functional form for $\Phi(\cdot)$ is different but quantitatively similar). Setting $L_{A}=L_{E}=0$ yields their results with closed capital accounts.

\subsection{Stability}

In the absence of any policy, the economy features a stochastic steady state around $\eta=0.41$, near where capital is best allocated at $\eta=0.35$ (since the AE has lower risk aversion), and the economy tends to drift toward the stochastic steady state after shocks move the system away. At the stochastic steady state, the AE's share of world capital is only slightly greater than $1 / 2$, so misallocation is minimal (at $\eta=0.41, \psi_{A} \approx 0.501$, so capital is almost efficiently allocated). Thus, the stationary distribution is concentrated at $\eta$ where flow output is relatively high (i.e., the economy is typically stable).

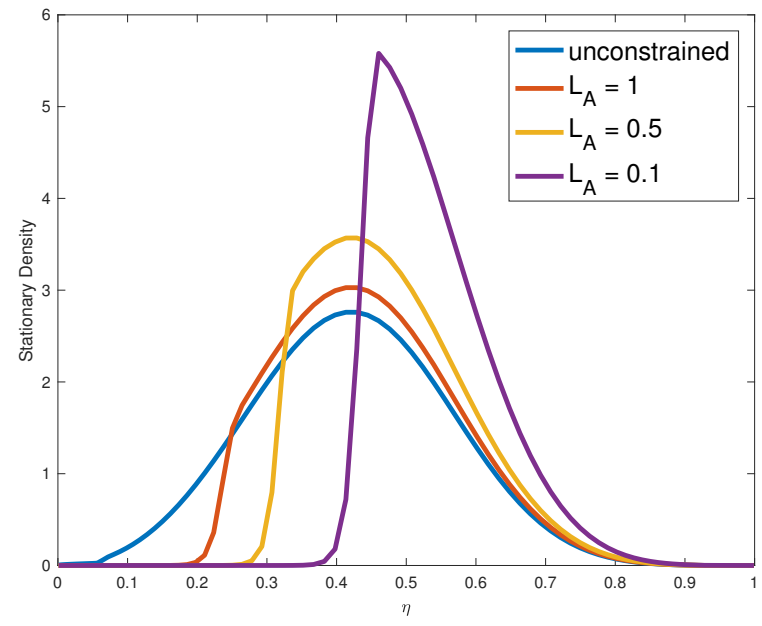

(a) EME unconstrained

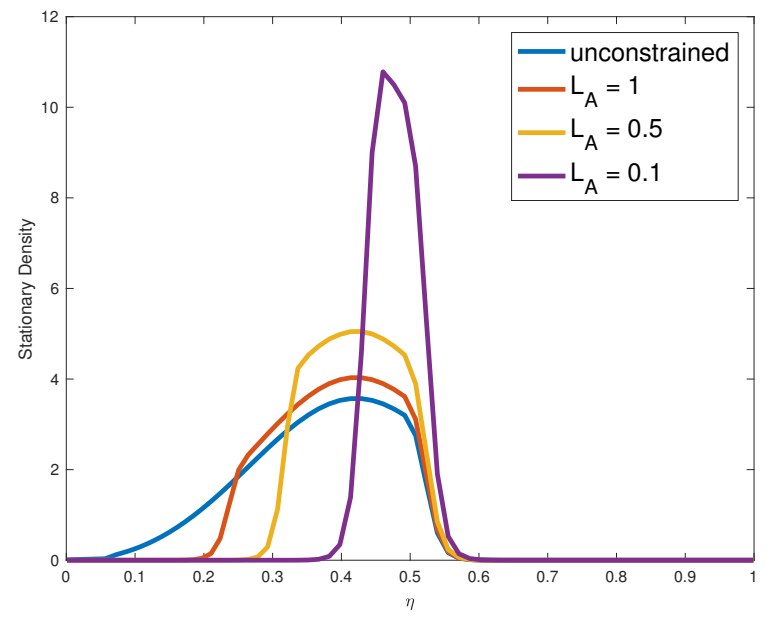

(b) $L_{E}=0$

Figure 1: Stationary distribution of $\eta$ (stability). Tighter constraints in the AE shift mass toward higher $\eta$ (good for the AE), and tighter constraints in the EME shift mass toward lower $\eta$ (good for the EME).

Figure 1 plots the stationary distribution of $\eta$ varying $L_{A}$ for two cases. Panel (a) considers when the EME does not impose leverage constraints, and Panel (b) considers the other extreme 
when the EME sets leverage to zero (closed capital account). The blue distribution in Panel (a) thus shows the distribution in the laissez-faire economy without constraints. Notably, it is the most disperse distribution. This is precisely the notion of long-run financial instability: the distribution of $\eta$ in the competitive equilibrium is more dispersed than any country would like. Moreover, this dispersion does not occur solely because of the level of endogenous volatility $\Sigma_{\eta}$.

Tighter regulation can shift mass in the direction a country prefers. Of course, the flip-side is that when the AE tightens, the relative wealth distribution must worsen from the perspective of the EME. Contrasting Panels (a) and (b) clearly illustrates how tightening by the EME has shifted the distribution toward low $\eta$ (bad for the AE).

Tighter leverage constraints increase the stability of the economy (in the sense of relative wealth differences) and reduce long-run financial stability because of two reasons. First, leverage decreases the volatility of $\eta$. Second, it improves the terms of trade after bad shocks, thus raising the rate at which net worth recovers. This "terms of trade hedge" increases the drift of $\eta$ near the stochastic steady state. These two forces together mean that following bad shocks which push the economy away from the steady state, the economy recovers at a faster rate with leverage constraints. In this way, the effect of capital controls in our model is quite complementary to the effect in Costinot et al. (2014). Because in our model taxing/limiting inflows improves the distribution of the economy, taxes on inflows need not be coupled with taxes on outflows. Figure 2 plots changes in the drift and volatility of $\eta_{t}$ (we define $\sigma_{\eta}=\sqrt{\sigma_{\eta A}^{2}+\sigma_{\eta E}^{2}}$ ). Panel (a) in Figure 3 illustrates the terms of trade hedge.

However, tighter regulation is not without cost. When constraints bind, flow allocations are worsened and output suffers. These costs are reflected by lower asset prices, as Figure 3.b) shows, and lower asset prices imply a lower growth rate of capital due to Tobin's q (9). Thus, tighter constraints lead to flow decreases in output and growth. As in Brunnermeier and Sannikov (2015), there is a dynamic trade-off between financial stability and the statically efficient allocation of capital as well as a trade-off between financial stability and growth.

These effects are present in Brunnermeier and Sannikov (2015), but in our model the distributional consequences are different. In both of our models, reducing leverage encourages financial 


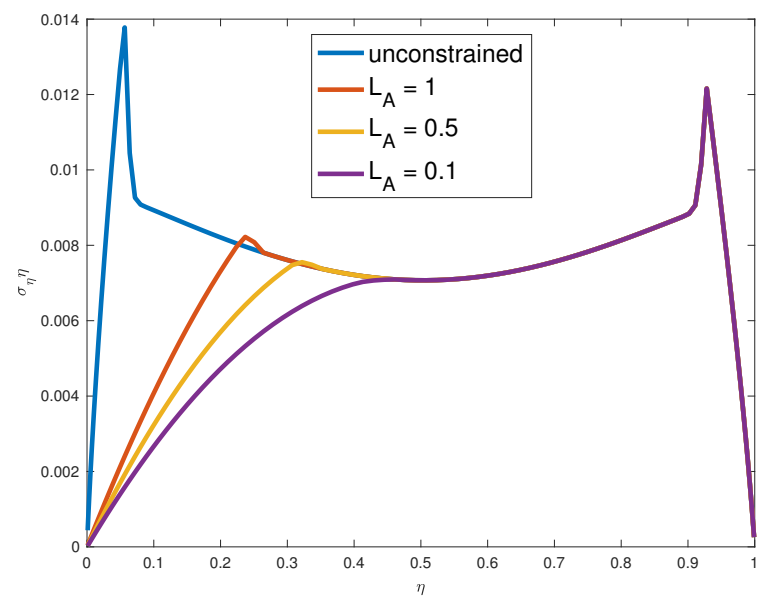

(a) Volatility $\sigma_{\eta}$

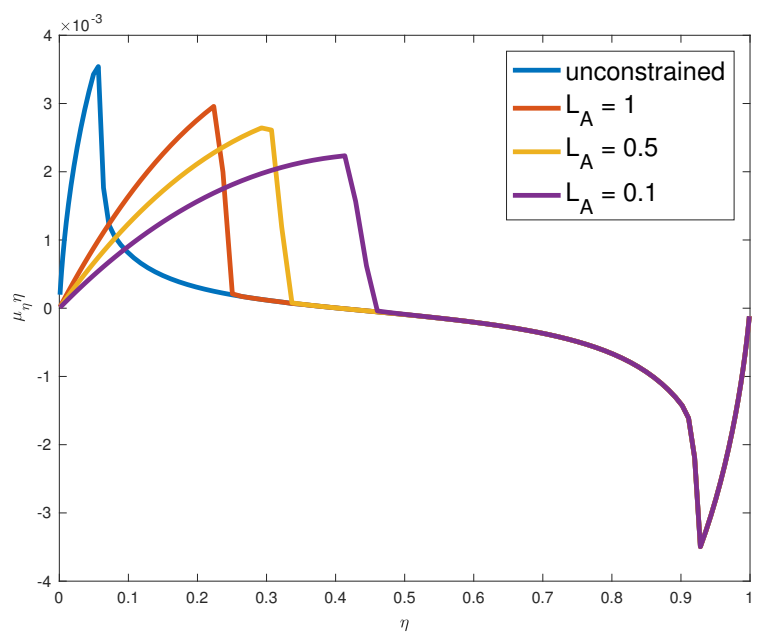

(b) Drift $\mu_{\eta}$

Figure 2: Evolution of $\eta$. Tighter constraints in the AE decrease volatility and (generally) increase drift, creating a more stable economy. (EME unconstrained)

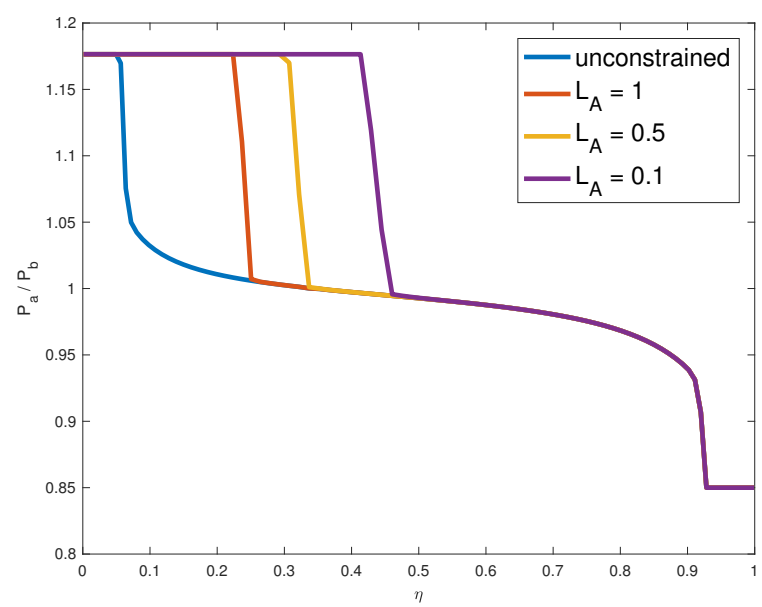

(a) Terms of Trade $P_{a, t} / P_{b, t}$

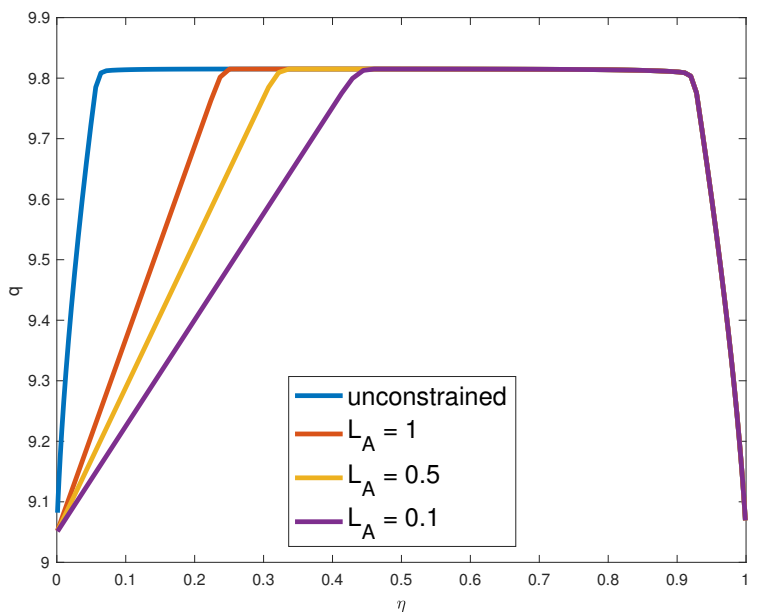

(b) Asset price $q_{t}$

Figure 3: Equilibrium Asset Price and Terms of Trade. (EME unconstrained)

stability by changing the time at which the terms of trade hedge applies. With open capital accounts, the terms of trade hedge for the AE is highest when $\eta$ is low, spurring faster growth when the AE has low net worth. However, this hedge in bad times comes at the cost of a higher probability of leaving the region near the stochastic steady state. In contrast, leverage constraints shift the terms of trade hedge so that it applies closer to the stochastic steady state. While tighter constraints 
decrease the drift of $\eta$ for low $\eta$, it increases the drift of $\eta$ near the stochastic steady state. In the long run, a higher drift closer to good times is better for stability, even though it takes longer to get out of bad times. The reason is that only an exceptionally bad sequence of shocks will cause $\eta$ to deviate far from the steady state. For most shocks, a higher drift of $\eta$ near the stochastic steady state prevents the economy from getting close to crisis. This mechanism is why closed capital accounts improve stability and welfare in Brunnermeier and Sannikov (2015) and in our model.

\subsection{Welfare}

The main goal of our analysis is to understand how countries choose regulation in order to maximize the welfare of its agents. In general, leverage constraints will not improve welfare almost everywhere. Consider the $\mathrm{AE}$ as an example. When $\eta$ is close to zero, restricting leverage slows down the rate at which agents rebuild their net worth. The reason is that expected returns will be very high but need to borrow to finance their investment. Limits on their leverage reduces their ability to capitalize on the high returns. As a result, the value function near $\eta=0$ will decrease when the leverage constraint tightens. However, if $\eta$ is further away from zero, then restrictions on leverage can promote financial stability enough that the value function increases with tighter constraints.

This non-monotonic response to leverage constraints across the state space complicates the welfare analysis of different regulatory regimes. Since countries are asymmetric, it does not make sense to simply assume equal initial wealth (i.e. $\eta_{0}=1 / 2$ ). The stochastic steady state may not be a reasonable initial condition either because the stationary distributions in Figure 1 indicate that the economy exhibits substantial long-run variance in $\eta{ }^{15}$ Ideally, we want to evaluate the welfare of the $\mathrm{AE}$ and EME in such a way that we capture the impact of leverage constraints on the long-run financial stability of the economy - the key motivation for these policy tools in the first place.

To address these issues, we apply an ex-ante measure of welfare. Rather than choose an arbitrary initial condition, we suppose that countries adopt a "prior" about the location of $\eta$ at time

\footnotetext{
${ }^{15}$ Another complication is the fact that welfare is non-monotonic with $\eta$. Consider the AE. For most of the state space, welfare increases with $\eta$. For high enough $\eta$, however, aggregate output is so low that the AE would actually prefer if $\eta$ was lower since the EME can produce good $b$ at a superior rate.
} 
$t=0$. Since neither country's information set "before time begins" should include a signal of $\eta_{0}$, the best "prior" is the stationary distribution of $\eta$. We therefore measure welfare by computing the expectation of Country $i$ 's value function with respect to the stationary distribution of $\eta$. We label this measure of welfare by $\mathbb{E}\left[V_{i}\right]$ and refer to it interchangeably as ex-ante welfare.

To make this notation more precise, recall that the value function for Country $i V_{i, 0}$ is subject to an initial condition $\eta_{0}$. We can reformulate this value function as the conditional expectation

$$
V_{i, 0}=\mathbb{E}\left[\int_{0}^{\infty} f^{i}\left(C_{i, t}, V_{i, t}\right) d t \mid \eta_{0}\right]
$$

Ex-ante welfare then is equivalent to integrating out the initial condition $\eta_{0}$ using some prior on its distribution.

We prefer this measure of welfare rather than evaluating the value functions at a single point because it captures the impact of regulation on long-run financial stability in two ways. First, conditional on $\eta_{0}$, leverage constraints influence the future investment opportunities which agents expect to have. For example, the stochastic steady state becomes more stable, so they expect capital to be optimally allocated more often. Second, they alter countries' uncertainty or beliefs about the economy's long-run stability. Evaluating countries' value functions only captures the first effect. The second effect, however, is important because a key benefit of greater long-run financial stability is increased certainty about the economy's health in its current state.

Welfare Spillovers. In this economy the dynamic benefits of a more stable economy generally outweigh the flow costs, and as a result tightening constraints in one country generally improves welfare for that country. However, there are important cross-country spillovers. Figure 4 plots ex-ante welfare for the $\mathrm{AE}$ as a function of the leverage constraint chosen by the EME, for three levels of constraints in the AE.

There are two important patterns to notice. First, given the policy in the EME, a tighter regulation in the $\mathrm{AE}$ (lower $L_{A}$ ) improves welfare in the AE. This is because the stability trade-off outweighs the flow cost, as discussed earlier. Second, fixing $L_{A}$ in the AE, welfare in the AE is increasing with $L_{E}$. In other words, tightening in the EME decreases welfare in the AE. Macro-level 


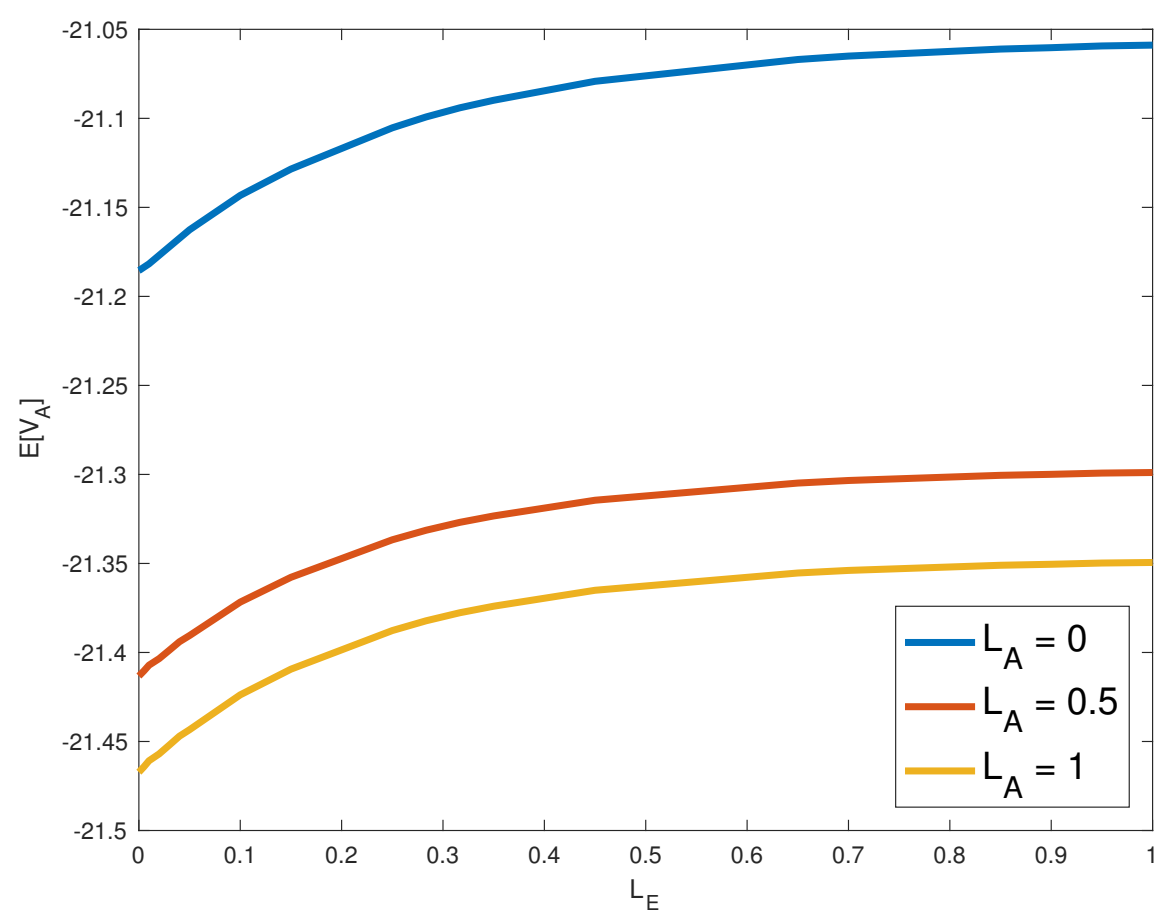

Figure 4: the AE's ex-ante welfare varying leverage constraints in the EME along the X-axis and in the $\mathrm{AE}$ across the different lines.

spillovers from capital account policies are reflected by changes in the stationary distribution of $\eta$ and in welfare.

There are two complementary intuitions for this result, and it is important to consider both. First, when the EME tightens, that shifts mass toward states when the AE is relatively poor. As we have seen, if the EME tightens and shifts mass toward states when the AE is poor, the AE can shift mass away from those states by tightening as well. Hence, uncoordinated regulation to improve stability has a "zero-sum" element: a country cannot shift mass away from $\eta=1$ without shifting it toward $\eta=0$. As we will see later, this mechanism is precisely why uncoordinated (Nash) policy choices will be tighter than policies coordinated by a social planner, since each country will have an incentive to tighten in order to improve the distribution of outcomes (from its perspective).

Second, more intermediation requires dealing with greater long-run financial instability. When a country takes on leverage (intermediating risk), it must also bear systemic risk (endogenous volatility) and a weakened terms of trade hedge, which produce greater financial instability. By restricting leverage, countries can reduce their exposure to sources of instability. These effects are 
reflected in Figures 2 and 3 . In the first figure, the volatility of $\eta$ decreases when the AE tightens regulation - and importantly, the volatility of $\eta$ decreases only at times when the AE is relatively poor, which is precisely when the $\mathrm{AE}$ does not want systemic risk. In the second figure, the terms of trade hedge applies much closer to the stochastic steady state, precisely when the AE needs it to maintain capital levels close to optimal. But as we've seen, limiting leverage in order to reduce instability is costly to the other country.

\section{Financial Asymmetry and Strategic Incentives}

In this section we let countries choose policies to maximize welfare in each country. Before diving into the results, we briefly clarify the policy game being played by countries. There are two players, the AE and the EME. They play a one-shot game. Before time $t=0$, each country chooses and commits to a fixed leverage constraint. They maximize their ex-ante welfare subject to constraints stated in Section 2, taking as given the other country's policy choice. We solve the coordinated equilibrium and the uncoordinated Nash equilibrium numerically. This "static game" illuminates some of the key forces governing how countries might engage in coordination without being complicated by the problems of multiple equilibria that emerge in dynamic and repeated games.

\subsection{Coordinated and Uncoordinated Policies}

Our numerical results indicate that closing capital accounts is not only a Nash equilibrium but a dominant strategy. Figure 5 plots ex-ante welfare in the AE, varying $L_{A}$ for a set of $L_{E}{ }^{16}$ What is clear is that for each $L_{E}$, welfare in the $\mathrm{AE}$ is maximized by $L_{A}=0$, and it monotonically increases as $L_{A}$ decreases. The same behavior holds for the EME.

Table 2 compares outcomes in the competitive equilibrium (no constraints) to the coordinated and uncoordinated equilibria using the ex-ante measure. Our first main result—-that uncoordinated

\footnotetext{
${ }^{16}$ This figure only displays a few cases. We determine that closed capital accounts is a dominant strategy in two ways. First, we compute a much finer grid of pairs of leverage constraints, which again suggests closed accounts are dominant strategies. Second, we conduct a fixed point iteration where we fix one player's constraint, compute the other player's best response, fix that best response, compute the first player's best response, and so on, considering many initial conditions. See Appendix C for a more thorough description.
} 


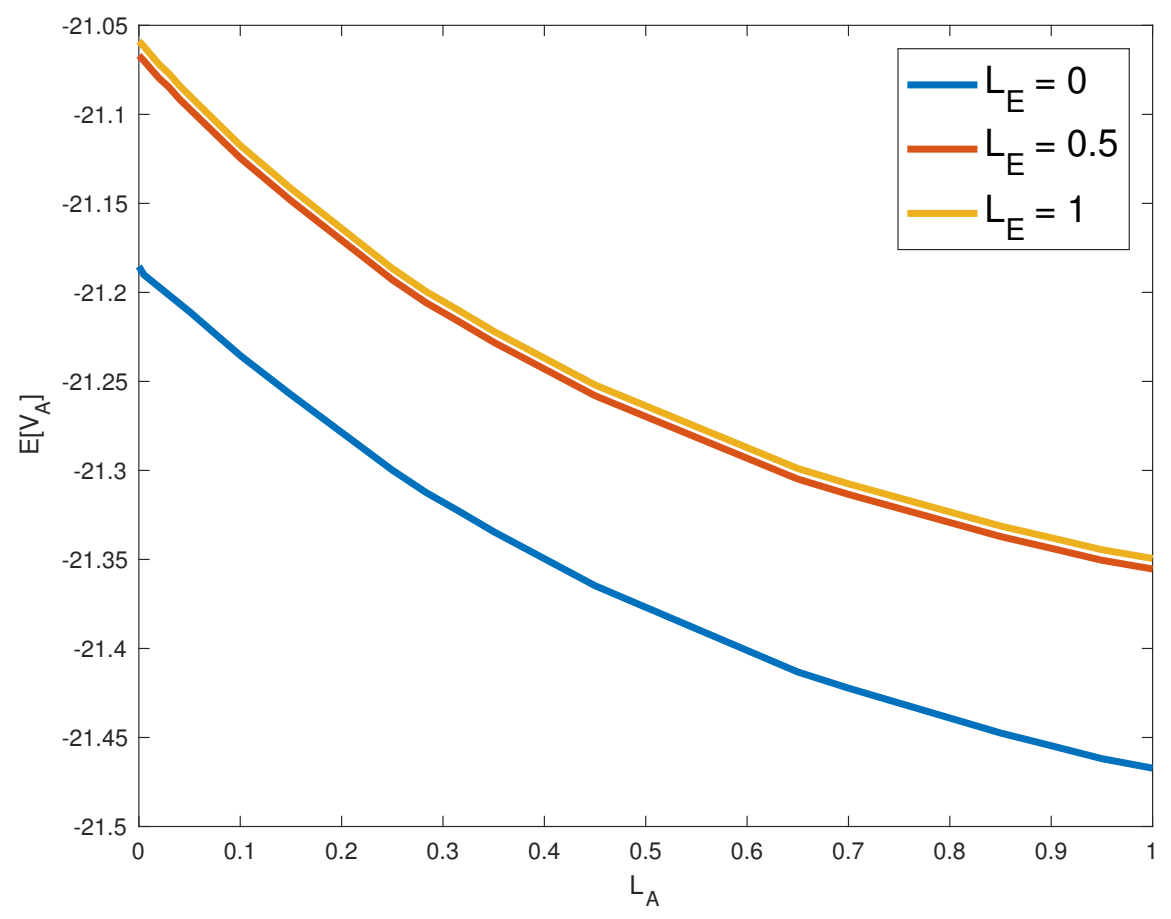

Figure 5: Welfare in the AE varying $L_{A}$ for given $L_{E}$. Setting $L_{A}=0$ appears to be a dominant strategy (i.e., best response to any $L_{E}$ ).

constraints are tighter than coordinated constraints—holds broadly across parameters, including in a symmetric model. For all parameters we have considered the Nash equilibrium is zero. While prudential regulations in our model are highly stylized, the key insight should apply across a wide range of potential environments. The key mechanism of our model is that, when international credit markets are imperfect, a policy that stabilizes the $\mathrm{AE}$ will lead the $\mathrm{AE}$ to be relatively richer more frequently. ${ }^{17}$ This is a negative spillover to the EME, which increases the incentive for the EME to enact stabilizing policies. While a richer setup is likely to generate positive spillovers through other sets of pecuniary externalities, we expect that the negative externality present in our model would continue to be present. Thus, while the quantitative importance of our mechanism will depend on the full set of global interactions, on the margin the mechanism we highlight will lead to tighter uncoordinated policies relative to global coordination.

\footnotetext{
${ }^{17}$ While in our model leverage limits are controls on capital inflows, in reality these instruments can have very different roles. Korinek and Sandri (2016) quantitatively find the optimal capital control and macroprudential regulation for emerging economies to mitigate contractionary exchange rate depreciations and reduce the amount and riskiness of financial liabilities. Nonetheless, the authors find it is optimal for emerging economies to employ both instruments in order to improve stability.
} 
Table 2: Consumption equivalent ex-ante welfare in percentage points relative to the competitive equilibrium with coordinated and uncoordinated leverage limits.

\begin{tabular}{ccccc}
\hline \hline & $L_{A}$ & $L_{E}$ & $\Delta$ Welfare for AE & $\Delta$ Welfare for EME \\
\hline \hline Social optimum & $177.4 \%$ & $0 \%$ & $-7.88 \%$ & $13.1 \%$ \\
Nash & 0 & 0 & $26.8 \%$ & $-7.62 \%$ \\
& & & & \\
\hline \hline
\end{tabular}

The second critical result which emerges in this analysis is that the EME is most hurt by a lack of policy coordination. The EME experiences a $13.1 \%$ welfare increase in consumption equivalent units in the social optimum relative to the competitive equilibrium but suffers a $7.62 \%$ consumption equivalent decrease in Nash equilibrium. In contrast, the low risk aversion AE benefits in Nash equilibrium compared to coordination (with equal Pareto weights) and especially compared to laissez faire. With our calibration, the $\mathrm{AE}$ gains $26.8 \%$ in consumption equivalents relative to the competitive equilibrium. If countries coordinate, then the AE actually suffers a $7.8 \%$ decrease in consumption equivalents 18 Hence, the AE benefits from lack of coordination (choosing tighter policy than the coordinated optimum) but the EME is hurt from lack of coordination (choosing essentially the same policy in each case) 19

Since countries are asymmetric, equal Pareto weights are not necessarily the correct choice. Moreover, it is instructive to consider the change in the AE's and EME's ex-ante welfare as a social planner varies Pareto weights. Toward this end, Figure 6 plots welfare pairs $\left(\mathbb{E}\left[V_{A}\right], \mathbb{E}\left[V_{E}\right]\right)$ for each country in different regulation regimes. The black asterisk is the welfare pair in competitive equilibrium (no constraints), the black circle is the welfare pair in Nash equilibrium (zero leverage), the red line is the Pareto frontier when the Pareto weight $\alpha$ is less than $1 / 2$, and the blue line is

\footnotetext{
${ }^{18}$ The asymmetry in welfare widens as the financial asymmetry widens. In other words, the larger the difference $\gamma_{E}-\gamma_{A}$ is, the more that the EME benefits from coordination and the more that the EME suffers in Nash equilibrium. See Table 3 .

${ }^{19}$ These welfare changes are implausibly large because we assume a high degree of market incompleteness (no international equity issuance). Adding more realistic risk sharing would decrease the quantitative significance of our analysis but would not change the qualitative story.
} 
the Pareto Frontier when $\alpha$ is greater than or equal to $1 / 220$ The Nash equilibrium is inside the frontier, though just barely. Thus, there exists a Pareto weight where the total welfare loss under Nash is not large, but the distributional consequences for the AE and EME are large.

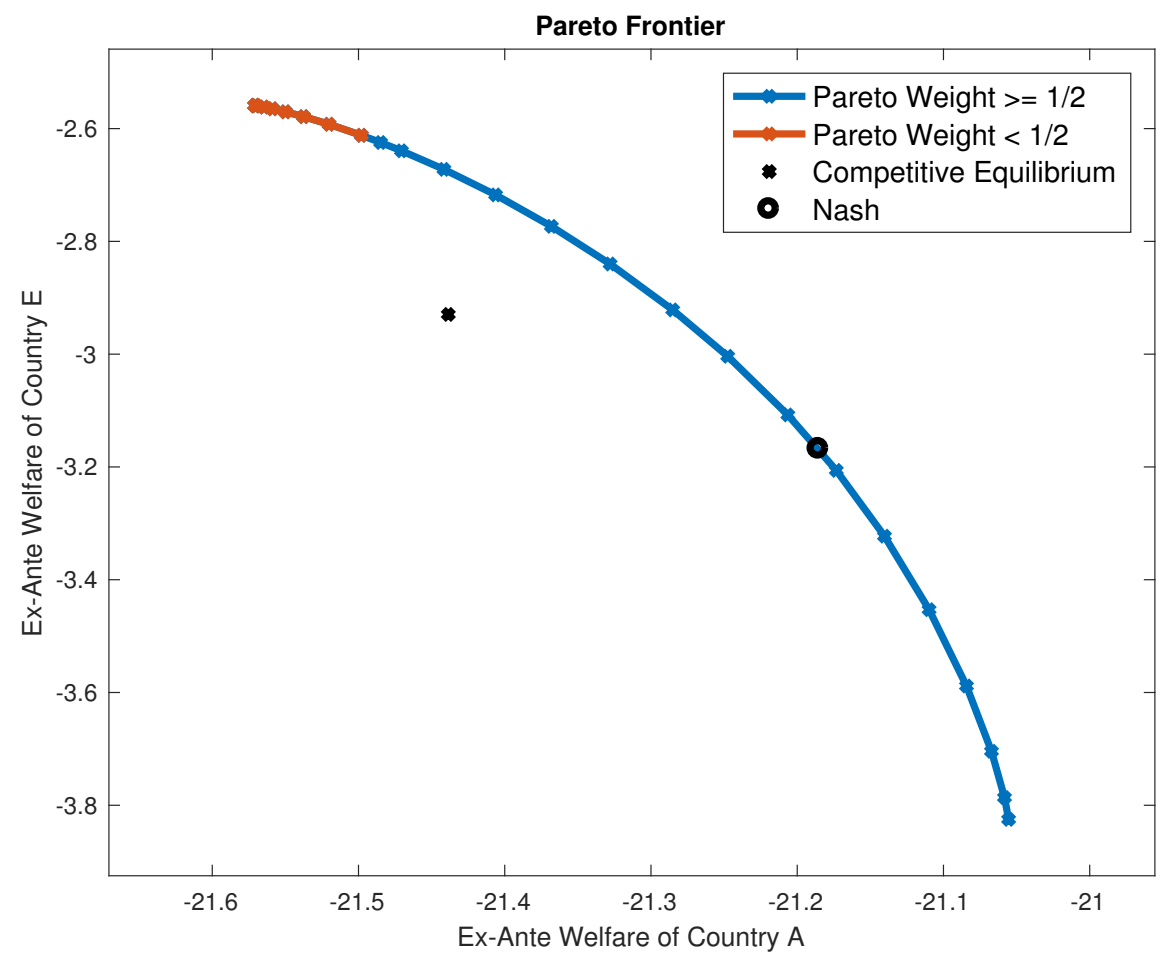

Figure 6: $\mathbb{E}\left[V_{E}\right]$ against $\mathbb{E}\left[V_{A}\right]$ as the Pareto weight $\alpha$ on the AE's ex-ante welfare varies from 0 to 1. The values from the competitive and Nash equilibria are also plotted.

Figure 7 plots the optimal leverage chosen by a global social planner as we vary the weight put on the AE. When all weight is put on the EME, the social planner calls for very high leverage by the $\mathrm{AE}$ and zero leverage for the EME. This is sensible: the social planner wants the AE to bear all risk (fundamental and systemic) and to provide intermediation to the EME. As more weight is put on the $\mathrm{AE}$, the social planner imposes tighter leverage in the $\mathrm{AE}$, thus protecting the $\mathrm{AE}$ from systemic risk. If the social planner cares only about the AE, then the EME will be called on to provide intermediation.

These results show the stark contrast in welfare relative to the competitive equilibrium when countries choose policies according to Nash: the AE benefits substantially while the EME is hurt.

\footnotetext{
${ }^{20}$ We construct the Pareto Frontier by computing optimal policies across 30 Pareto weights ranging equidistantly from near zero to near one. The hashes in the red and blue lines indicate particular Pareto weights.
} 


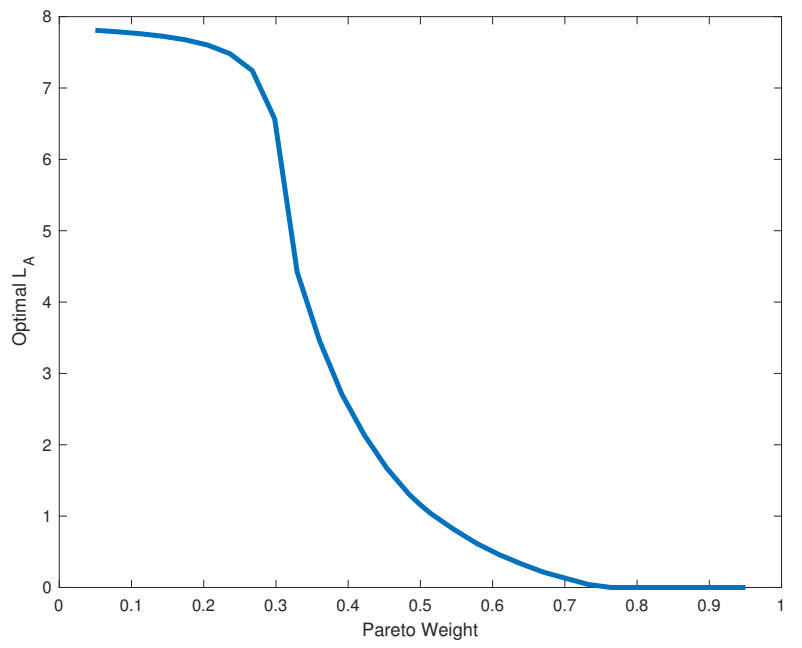

(a) AE Leverage

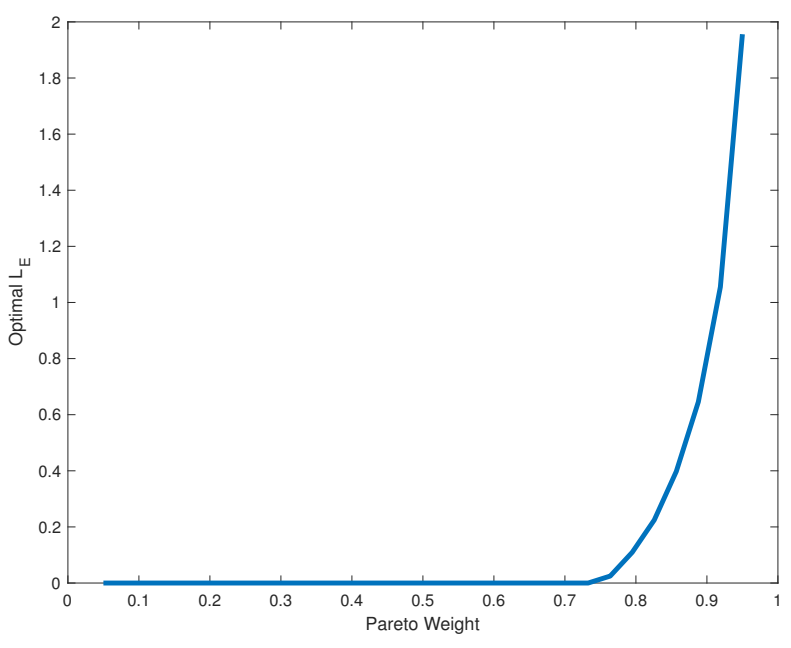

(b) EME Leverage

Figure 7: Optimal Leverage Varying the Pareto Weight on the AE.

With weights approximately in the interval $[0.6,0.7]$, the policy chosen by a planner will Pareto dominate the allocation in the competitive equilibrium. The Nash equilibrium appears to be on or near the Pareto frontier when $\alpha$ is sufficiently large. When the planner puts non-trivial weight on the EME, then it is optimal for the AE to intermediate risk (i.e., to bear financial instability) for the sake of the EME. However, this requires the AE to suffer worse welfare outcomes. Without coordination the $\mathrm{AE}$ is not willing to bear the financial risk that benefits the rest of the world.

Moreover, it is likely that any policy coordiation would result in policies similar to those chosen by a social planner who places a large Pareto weight on the AE. The location of Nash equilibrium in Figure 6 indicates that Pareto-improving policy coordination would produce very small gains (just inside the frontier), and the outcome would resemble that of a social planner who chooses a fairly large $\alpha$. Even if the AE is willing to accept worse welfare outcomes, a priori one would expect the consequent policies to still be fairly close to Nash equilibrium. If coordination requires the AE to give up too much welfare, then the AE can always unilaterally set $L_{A}=0$. Coordinating prudential regulations will necessitate policies that are strongly determined by the AE's welfare. 


\subsection{Mechanisms}

Here, we investigate the mechanisms driving our results. First, we consider a benchmark case where we shut off the terms of trade hedge by assuming countries directly produce the final consumption good. This case illustrates the effect of leverage constraints on the pricing of risk in equilibrium. Second, we consider another benchmark case where we shut off amplification through asset prices by assuming countries have access to a linear investment technology, which causes the price of capital to be constant. This case focuses attention on the terms of trade hedge. Finally, we construct counterfactual laws of motion to compare the relative importance of risk pricing and the terms of trade in our baseline model. Due to space constraints, we only briefly describe the benchmark model variants and refer the reader to Appendix B for more details.

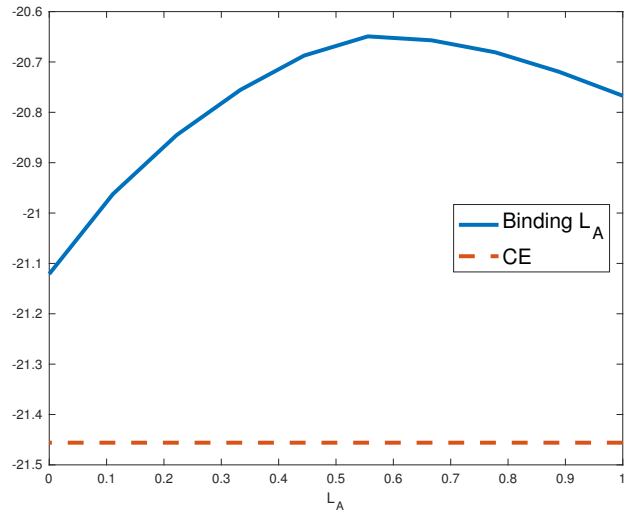

(a) One Good (No ToT hedge)

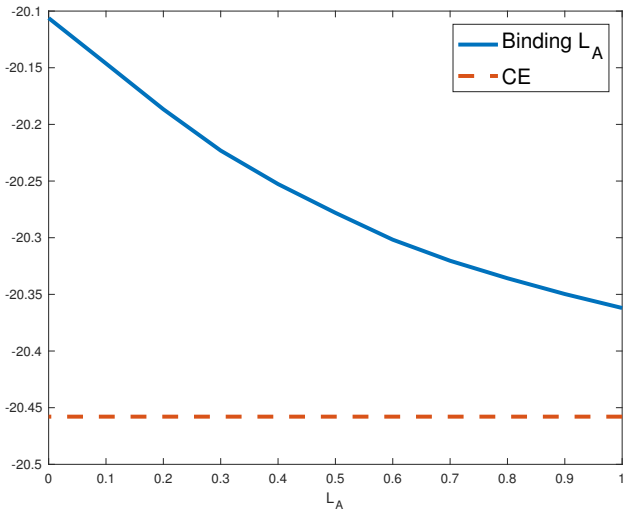

(b) Linear Investment Technology (Constant asset price $q$ )

Figure 8: Panels (a) and (b) plot the ex-ante welfare of the AE in the one good model and in the linear investment technology model, respectively. The EME imposes $L_{E}=0$. The blue line plots the AE's ex-ante welfare with a binding but varying leverage constraint while the dashed red line plots the AE's ex-ante welfare if it does not impose a binding leverage constraint.

Risk Pricing Only (no terms of trade hedge). Intermediate goods $a$ and $b$ no longer exist in this benchmark. Instead, agents in the AE produce the final consumption good at rate $\bar{a} k_{t}$ while agents in the EME produce the final consumption good at rate $\underline{a} k_{t}$, where $\bar{a}>\underline{a}$. This modification removes the terms-of-trade hedge that promotes the rate at which net worth recovers precisely 
when a country becomes relatively poor ${ }^{21}$ Therefore, if leverage constraints still affect welfare, then the reason is that they alter the pricing of risk in equilibrium and influence the buildup of systemic risk. Figure 8(a) shows how tighter leverage constraints affect the AE's ex-ante welfare, assuming the EME imposes $L_{E}=0$. While restricting leverage improves ex-ante welfare relative to the competitive equilibrium, it is no longer monotonic. Instead, it is optimal to permit some leverage. Thus, while leverage constraints have a meaningful impact on welfare through their effect on equilibrium risk pricing alone, their benefits do not justify completely closed capital accounts.

Intuitively, the static benefit of efficiently allocating capital is more pronounced when no terms of trade hedge applies. Without a terms of trade in intermediate goods, countries only compare the gains from higher output against the gains of lower asset price volatility. In the full model, tighter leverage constraints provide an additional benefit through a better terms-of-trade hedge. The economy becomes stabler in terms of its volatility and the rate at which countries rebuild their net worth.

Terms of Trade Only (constant asset price). We choose a new functional form for the internal investment technology:

$$
\Phi(\imath)=\lambda \imath
$$

This linear technology implies a constant price of capital $q=1 / \lambda$, so there is no volatility in the price of capital. We choose $\lambda$ to match the average price of capital in our original model. The only source of endogenous risk now is from countries' leverage choices. Figure 8 (b) shows that it remains optimal for the AE to close its capital accounts, and ex-ante welfare continues to monotonically increase as $L_{A}$ decreases. Moreover, we verify that, fixing $L_{A}=0$, the EME's welfare monotonically increases when $L_{E}$ decreases, hence $\left(L_{A}, L_{E}\right)=(0,0)$ is still a Nash equilibrium.

\footnotetext{
${ }^{21}$ In principle, the ideal comparison is setting $\bar{a}=\underline{a}$, but it turns out that in this equilibrium asset prices become constant, and neither country borrows. In addition, Brunnermeier and Sannikov (2015) shows the stationary distribution is degenerate. By assuming one country is permanently more productive, we can obtain a stationary equilibrium with fire sales but without the terms of trade providing a hedge in bad times. While this benchmark does not perfectly isolate the terms of trade hedge, it is still informative because it does shut off the hedge.
} 
The fact that we obtain these results pinpoints the terms of trade hedge as the key mechanism driving our results.

For intuition, recall that the log of a geometric Brownian motion $G_{t}$ with drift $\mu$ and volatility $\sigma$ can be solved in closed form as

$$
\log \left(G_{t}\right)=\log \left(G_{0}\right)+\left(\mu-\frac{\sigma^{2}}{2}\right) t+\sigma W_{t}
$$

A change in $\mu$ has a "first-order" effect on $\mathbb{E}\left[\log \left(G_{t}\right)\right]$, but a change in $\sigma$ only has a "secondorder" effect since it is the variance that directly affects $\mathbb{E}\left[\log \left(G_{t}\right)\right]$. For example, if $\mu$ and $\sigma$ both decreased by one percentage point, the change in $\mu$ would have a larger effect (assuming $\sigma<1$ ). The behavior of $\eta_{t}$ is similar. We can heuristically rewrite (16), the law of motion for $\eta$, as

$$
\begin{aligned}
\frac{d \eta_{t}}{\eta_{t}}= & (\text { Dividend Yield }+ \text { Covariance Terms }) d t \\
& + \text { Leverage Ratio } \times(\text { Fundamental }+ \text { Endogenous Vol. }) d \mathbf{W}_{t} .
\end{aligned}
$$

An increase in the terms of trade hedge increases the dividend yield and thus has a "first-order" effect because it directly affects the drift of $\eta$. Although a decrease in asset price volatility influences the drift of $\eta$, the effect occurs through covariances and is therefore still "second-order."

In Appendix B.3 we examine the relative contributions risk pricing and the terms of trade hedge from another perspective by considering "counterfactual" scenarios in which we replace any prices of intermediate goods in the expression for $\mu_{\eta}$ in $(16$ by the prices from the constrained equilibrium (thus we suppose the only effect of prudential limits is on terms of trade and not also on endogenous volatility). These results show that the terms of trade hedge is the more important mechanism through which leverage constraints provide financial stability.

Incomplete Markets and the Terms of Trade Although the effect from the terms of trade dominates the effect from endogenous risk, incomplete markets are still the key reason why the terms of trade matter. As we noted in Section 2, if markets were complete, then countries would not strategically manipulate capital flows to influence the terms of trade. Incomplete markets, how- 
ever, force countries to hold portfolios where their production as a share of world output fluctuates. In equilibrium, their production is more valuable due to higher excess returns precisely when their net worth is low. This feature creates an incentive to dynamically manipulate the terms of trade between goods $a$ and $b$. In other words, incomplete markets cause time variation in a country's income which creates similar incentives for terms-of-trade manipulation to those studied in Costinot et al. (2014).

However, in contrast to previous work on terms-of-trade manipulation, prudential regulations affect the terms of trade through portfolio choice and production decisions, not through consumption behavior. With unit EIS, countries always consume a constant fraction of their share of world wealth (e.g., for the $\mathrm{AE}, C_{A}=\rho q \eta$ ). Agents consume more or less as a result of policy only due to changes in $q$. Limiting leverage instead affects the scale of a country's production, which influences the returns a country receives from holding capital. Because individual agents take prices as given, they do not internalize how their leverage decisions change their terms of trade. By limiting leverage, countries earn better returns on capital, which speeds up the rate at which their net worth recovers after bad shocks near the stochastic steady state.

\subsection{Discussion}

While our model is highly stylized, these results should still hold in some form across a wide range of potential environments. For example, our results are robust to considering other ways of modeling AEs as the financial center. We obtain qualitatively identical results if AEs and EMEs have identical preferences but AEs face lower fundamental volatility for their investments. What matters for our results is that AEs have an incentive to bear global risk but financial instability caused by market incompleteness encourages them to, at least in part or on the margin, regulate that risk away. This incentive complicates the conduct of policy coordination which grants EMEs greater access to financial intermediation by AEs. To achieve coordination, EMEs may need to compensate AEs in some form in order for AEs to agree to bear more global risk. If EMEs have strong demand for additional intermediation, then AEs may be able to obtain substantial compensation as the price for coordination. 
We have abstracted away from channels for positive macro-level spillovers, and richer set-ups would likely incorporate them. However, the key insight of our model is that a stabilizing policy in one country means that country becomes relatively richer more frequently, and we expect this mechanism, on the margin, to remain in models with positive externalities. A more realistic Nash outcome would likely involve tighter leverage constraints relative to the coordinated equilibrium, but constraints would perhaps be positive rather than zero. However, since the EME would still want the AE to provide financial intermediation, any policy coordination will continue to place substantial weight on the AE's welfare. For the interested reader, Appendix D discuss some implications of our model for international financial regulations.

Several dimensions of our model can be further extended to provide greater insight as to how global macroprudential and capital account regulation should be conducted. Our analysis ignores any important heterogeneity within countries. Indeed, Buch and Goldberg (2016) find that heterogeneity among banks lead to different responses to cross-border spillovers. Thus, a homogenous constraint may not be the best choice, and further research should be conducted to determine how agent heterogeneity influences the optimal constraint. Second, including richer heterogeneity across countries (besides asymmetric risk aversions), and particularly across financial sectors, would likely provide additional forces for strategic interactions between countries.

\section{Conclusion}

Emerging market economies depend on "center" countries in the global financial system to provide intermediation and risk-bearing, but to do so requires that AEs bear systemic risk. When international credit markets are imperfect, more stable countries are more likely to be relatively wealthy compared to less stable countries. Prudential regulations, when effective, increases economic stability, creating a macro-level spillover through international capital flows. As a result, tight prudential controls in one country provides strategic incentives for tight policy in the other. Because countries can unilaterally choose their prudential regulations, AEs have an incentive to provide less intermediation than demanded by EMEs. Therefore, coordination will likely result in policies that place relatively more emphasis on the outcomes of AEs than the outcomes of EMEs. 


\section{References}

ACHARYA, S. AND J. BENGUi (2018): "Liquidity traps, capital flows," Journal of International Economics, 114, 276-298.

AChARYA, V. V. (2013): "The Dodd-Frank Act and Basel III: Intentions, Unintended Consequences, and Lessons for Emerging Markets," in New Paradigms for Financial Regulation: Emerging Market Perspectives, ed. by M. Kawai and E. S. Prasad, Washington, D.C.: Brookings Institution Press.

Agénor, P., L. Gambacorta, E. Kharroubi, G. Lombardo, and L. P. da Silva (2018): "Assessing the gains from international macroprudential policy cooperation," BIS Working Papers No 643.

Alford, D. (2013): "International Financial Reforms: Capital Standards, Resolution Regimes, and Supervisory Colleges and Their Effect on Emerging Markets," in New Paradigms for Financial Regulation: Emerging Market Perspectives, ed. by M. Kawai and E. S. Prasad, Washington, D.C.: Brookings Institution Press, chap. 3, 244-278.

Aoki, K., G. Benigno, And N. KiYotaki (2009): “Capital flows and asset prices," in NBER International Seminar on Macroeconomics 2007, University of Chicago Press, 175-216.

- (2010): “Adjusting to capital account liberalization,” Tech. rep., CEP Discussion Paper No 1014.

BAIR, S. (2012): Bulls by the Horn: Fighting to Save Main Street from Wall Street and Wall Street from Itself, Free Press.

Banerjee, R., M. B. Devereux, And G. Lombardo (2016): "Self-oriented monetary policy, global financial markets and excess volatility of international capital flows," Journal of International Money and Finance, 68, 275-297.

BARTh, J. R., G. CAPRIO JR, AND R. LeVine (2013): "Bank Regulation and Supervision in 180 Countries from 1999 to 2011," Journal of Financial Economic Policy, 5, 111-219.

BECK, T. (2018): "Basel III and Unintended Consequences for Emerging Markets and Developing Economies - Part 5: Effects on Capital Market Development and the Real Economy,”.

BECK, T., E. JONES, AND N. WoOdS (2018): "Developing countries navigating global banking standards," https://www.geg.ox.ac.uk/project/developing-countries-navigating-global-bankingstandards. 
BiAnCHI, J. (2010): "Credit Externalities: Macroeconomic Effects and Policy Implications," American Economic Review, 100, 398-402.

- (2011): “Overborrowing and Systemic Externalities in the Business Cycle," American Economic Review, 101, 3400-3426.

Bianchi, J. And E. G. Mendoza (2010): “Overborrowing, Financial Crises and 'Macroprudential' Taxes," Working Paper 16091, National Bureau of Economic Research.

Boar, C., L. Gambacorta, G. Lombardo, and L. A. Pereira da Silva (2017): "What are the effects of macroprudential policies on macroeconomic performance?" BIS Quarterly Review.

Bolton, P. And D. S. Scharfstein (1990): "A Theory of Predation Based on Agency Problems in Financial Contracting," The American Economic Review, 80, pp. 93-106.

BRUnNermeier, M. K. AND Y. SAnnikov (2014a): "A Macroeconomic Model with a Financial Sector," American Economic Review, 104, 379-421.

(2014b): “A Macroeconomic Model with a Financial Sector," American Economic Review, $104,379-421$.

(2015): "International Credit Flows and Pecuniary Externalities," American Economic Journal: Macroeconomics, 7, 297-338.

- (2016a): “The I theory of money,” Tech. rep., National Bureau of Economic Research.

(2016b): "Macro, money, and finance: A continuous-time approach," in Handbook of Macroeconomics, Elsevier, vol. 2, 1497-1545.

BuCH, C. M. AND L. GoldBERG (2016): “Cross-border prudential policy spillovers: how much? How important? Evidence from the International Banking Research Network," Tech. rep., National Bureau of Economic Research.

Caballero, R. J. And A. Krishnamurthy (2001): "International and domestic collateral constraints in a model of emerging market crises," Journal of Monetary Economics, 48, 513548.

(2004): “Smoothing sudden stops,” Journal of Economic Theory, 119, 104-127.

CAballero, R. J. And A. SimseK (2016): "A Model of Fickle Capital Flows and Retrenchment: Global Liquidity Creation and Reach for Safety and Yield," Tech. rep., National Bureau of Economic Research. 
(2017): "A risk-centric model of demand recessions and macroprudential policy," Tech. rep., National Bureau of Economic Research.

Costinot, A., G. Lorenzoni, AND I. Werning (2014): "A theory of capital controls as dynamic terms-of-trade manipulation," Journal of Political Economy, 122, 77-128.

Davis, J. S. And M. B. Devereux (2019): "Capital Controls as Macro-Prudential Policy in a Large Open Economy," NBER Working Paper Series 25710, National Bureau of Economic Research.

Di Tella, S. (2017): "Uncertainty Shocks and Balance Sheet Recessions," Journal of Political Economy, 125, 2038 - 2081.

DreZner, D. W. (2007): All Politics is Global: Explaining International Regulatory Regimes, Princeton University Press.

DufFIE, D. AND L. G. EPSTEIN (1992): "Stochastic differential utility," Econometrica: Journal of the Econometric Society, 353-394.

FARHI, E. AND I. WERnING (2014): "Dilemma not trilemma? Capital controls and exchange rates with volatile capital flows," IMF Economic Review, 62, 569-605.

GÂrleanu, N., S. PAnageas, And J. Yu (2015): "Financial entanglement: A theory of incomplete integration, leverage, crashes, and contagion," American Economic Review, 105, 19792010.

GOURINCHAS, P.-O. AND H. REY (2007): "From world banker to world venture capitalist: US external adjustment and the exorbitant privilege," in $G 7$ current account imbalances: sustainability and adjustment, University of Chicago Press, 11-66.

Gourinchas, P.-O., H. Rey, N. Govillot, et AL. (2010): "Exorbitant privilege and exorbitant duty," Tech. rep., Institute for Monetary and Economic Studies, Bank of Japan.

He, Z. And A. Krishnamurthy (2012): "A Model of Capital and Crises," The Review of Economic Studies, 79, 735-777.

_ (2013): “Intermediary Asset Pricing," American Economic Review, 103, 732-70.

(2014): “A Macroeconomic Framework for Quantifying Systemic Risk," Tech. rep., National Bureau of Economic Research. 
Heathcote, J. And F. PerRi (2013): “The International Diversification Puzzle Is Not as Bad as You Think," Journal of Political Economy, 121, 1108 - 1159.

- (2016): “On the desirability of capital controls," IMF Economic Review, 64, 75-102.

Holmström, B. And J. TiRole (1997): "Financial Intermediation, Loanable Funds, and the Real Sector," The Quarterly Journal of Economics, 112, 663-91.

Jensen, M. C. And W. H. Meckling (1976): "Theory of the firm: Managerial behavior, agency costs and ownership structure," Journal of Financial Economics, 3, 305 - 360.

JiN, H. AND H. SHEN (2019): "Foreign asset accumulation among emerging market economies: A case for coordination," Review of Economic Dynamics.

KELLER, L. (2018): "Capital controls and risk misallocation: evidence from a natural experiment," Available at SSRN 3099680.

KORINEK, A. (2016): "Currency wars or efficient spillovers? A general theory of international policy cooperation," Tech. rep., National Bureau of Economic Research.

KORINEK, A. AND D. SANDRI (2016): “Capital controls or macroprudential regulation?” Journal of International Economics, 99, 27-42.

LAne, P. R. AND G. M. Milesi-Ferretti (2007): “The external wealth of nations mark II: Revised and extended estimates of foreign assets and liabilities, 1970-2004," Journal of international Economics, 73, 223-250.

MagGiori, M. (2017): "Financial intermediation, international risk sharing, and reserve currencies," American Economic Review, 107, pp. 3038-71.

MendozA, E. G. (2010): “Sudden Stops, Financial Crises, and Leverage," American Economic Review, 100, 1941-66.

Phelan, G. (2016): "Financial intermediation, leverage, and macroeconomic instability," American Economic Journal: Macroeconomics, 8, 199-224.

SergeyeV, D. (2014): “Financial Integration and Financial Instability,” .

ShimizU, Y. (2013): "Global Financial Regulations and the Asian Financial System: Lessons from the Financial Crisis," in New Paradigms for Financial Regulation: Emerging Market Perspectives, ed. by M. Kawai and E. S. Prasad, Washington, D.C.: Brookings Institution Press.

TAYLOR, M. (2010): "Basel III is bad news for emerging economies," Financial Times. 
Walter, A. (2015): “Emerging Countries and Basel III: Why is Engagement Still Low?" New Thinking and the New G20 Series 4, Centre for International Governance Innovation.

WATANAGASE, T. (2013): "The Impact of Changes in the Global Financial Regulatory Landscape on Emerging Markets," in New Paradigms for Financial Regulation: Emerging Market Perspectives, ed. by M. Kawai and E. S. Prasad, Washington, D.C.: Brookings Institution Press, chap. 3, 225-243.

White, W. R. (2001): “The evolving global financial system: some implications for emerging markets," Keynote lecture by William R. White, (former) Economic Adviser for the Bank for International Settlements (BIS), delivered at the Indira Gandhi Institute in Mumbai, India.

\section{Appendices for Online Publication}

\section{A Proofs}

\section{A.1 Asset Pricing Equations}

First, consider the Hamilton-Jacobi-Bellman (HJB) equation for the AE agents:

$$
0=\max _{c_{A, t}, x_{A a, t}, x_{A b, t}} f\left(c_{A, t}, V_{A, t}\right)+\mathbb{E}_{t}\left[d V_{A, t}\right]
$$

subject to the leverage constraint $L_{A, t}$ and the law of motion for net worth 12 . For now, suppress the max operator for economy of presentation. By Ito's product rule and Ito's lemma,

$$
\begin{aligned}
\mathbb{E}\left[d\left(\xi_{t} n_{A, t}\right)\right] & =\xi_{t} n_{A, t}\left(\mu_{\xi, t}+\mu_{n A, t}+\Sigma_{\xi, t} \cdot \Sigma_{n A, t}\right) \\
\mathbb{E}\left[\frac{d\left(\xi_{t} n_{A, t}\right)^{1-\gamma_{A}}}{1-\gamma_{A}}\right] & =\left(\xi_{t} n_{A, t}\right)^{-\gamma_{A}} \mathbb{E}\left[d\left(\xi_{t} n_{A, t}\right)\right]-\frac{\gamma_{A}}{2}\left(\xi_{t} n_{A, t}\right)^{-1-\gamma_{A}}\left(d\left(\xi_{t} n_{A, t}\right)\right)^{2} \\
& =\left(\xi_{t} n_{A, t}\right)^{1-\gamma_{A}}\left(\mu_{\xi, t}+\mu_{n A, t}+\Sigma_{\xi, t} \cdot \Sigma_{n A, t}\right)-\frac{\gamma_{A}}{2}\left(\xi_{t} n_{A, t}\right)^{1-\gamma_{A}}\left\|\Sigma_{\xi, t}+\Sigma_{n A, t}\right\|^{2}
\end{aligned}
$$


Plugging this quantity into the HJB yields

$$
\begin{aligned}
0= & \rho\left(1-\gamma_{A}\right) \frac{\left(\xi_{t} n_{A, t}\right)^{1-\gamma_{A}}}{1-\gamma_{A}}\left[\log \left(c_{A, t}\right)-\frac{1}{1-\gamma_{A}} \log \left(\left(\xi_{t} n_{A, t}\right)^{1-\gamma_{A}}\right)\right] \\
& +\left(\xi_{t} n_{A, t}\right)^{1-\gamma_{A}}\left(\mu_{\xi, t}+\mu_{n A, t}+\Sigma_{\xi, t} \cdot \Sigma_{n A, t}\right)-\frac{\gamma_{A}}{2}\left(\xi_{t} n_{A, t}\right)^{1-\gamma_{A}}\left\|\Sigma_{\xi, t}+\Sigma_{n A, t}\right\|^{2}
\end{aligned}
$$

Cancelling by $\left(\xi_{t} n_{A, t}\right)^{1-\gamma_{A}}$ and plugging in for the drift of the AE's net worth yields

$$
\begin{aligned}
0= & \rho\left[\log \left(c_{A, t}\right)-\frac{1}{1-\gamma_{A}} \log \left(\left(\xi_{t} n_{A, t}\right)^{1-\gamma_{A}}\right)\right]+\mu_{\xi, t}+\Sigma_{\xi, t} \cdot \Sigma_{n A, t} \\
& +\left[d r_{t}^{F}+\mathbb{E}\left[x_{A a, t}\left(d r_{t}^{A a}-d r_{t}^{F}\right)+x_{A b, t}\left(d r_{t}^{A b}-d r_{t}^{F}\right)\right]-\frac{c_{A, t}}{n_{A, t}}\right] \\
& -\frac{\gamma_{A}}{2}\left(\left\|\Sigma_{\xi, t}\right\|^{2}+2 \Sigma_{\xi, t} \cdot \Sigma_{n A, t}+\left\|\Sigma_{n A, t}\right\|^{2}\right) .
\end{aligned}
$$

Additionally plugging in for $\Sigma_{n A, t}$ obtains

$$
\begin{aligned}
0= & \rho\left[\log \left(c_{A, t}\right)-\frac{1}{1-\gamma_{A}} \log \left(\left(\xi_{t} n_{A, t}\right)^{1-\gamma_{A}}\right)\right]+\mu_{\xi, t} \\
& +\left[d r_{t}^{F}+\mathbb{E}\left[x_{A a, t}\left(d r_{t}^{A a}-d r_{t}^{F}\right)+x_{A b, t}\left(d r_{t}^{A b}-d r_{t}^{F}\right)\right]-\frac{c_{A, t}}{n_{A, t}}\right] \\
& +\left(1-\gamma_{A}\right) \Sigma_{\xi, t} \cdot\left[\left(x_{A a, t}+x_{A b, t}\right) \Sigma_{A a, t}\right] \\
& -\frac{\gamma_{A}}{2}\left(\left\|\Sigma_{\xi, t}\right\|^{2}+\left\|\left(x_{A a, t}+x_{A b, t}\right) \Sigma_{A a, t}\right\|^{2}\right) .
\end{aligned}
$$

Differentiating with respect to $c_{A, t}$ yields

$$
\rho\left(\xi_{t} n_{A, t}\right)^{1-\gamma_{A}} c_{A, t}^{-1}-\left(\xi_{t} n_{A, t}\right)^{1-\gamma_{A}} n_{A, t}^{-1}=0 \Rightarrow \frac{c_{A, t}}{n_{A, t}}=\rho .
$$

Differentiating w.r.t. to the portfolio shares on capital funded by inside equity yields, when leverage constraints are not binding,

$$
\begin{aligned}
& \mathbb{E}\left[d r_{t}^{A a}\right]-d r_{t}^{F}=\gamma_{A}\left(x_{A a, t}+x_{A b, t}\right) \Sigma_{A a, t} \cdot \Sigma_{A a, t}+\left(\gamma_{A}-1\right) \Sigma_{\xi, t} \cdot \Sigma_{A a, t}, \\
& \mathbb{E}\left[d r_{t}^{A b}\right]-d r_{t}^{F} \leq \gamma_{A}\left(x_{A a, t}+x_{A b, t}\right) \Sigma_{A a, t} \cdot \Sigma_{A a, t}+\left(\gamma_{A}-1\right) \Sigma_{\xi, t} \cdot \Sigma_{A a, t} .
\end{aligned}
$$


Note that the second inequality arises because the AE specializes in the production of good $a$.

Let $\varsigma_{A a, t}$ denote the covariance between the AE's volatility of holding capital and its volatility of net worth, and let $\zeta_{\xi A, t}$ denote the covariance between the volatility of $\xi$ and the AE's volatility of holding capital. These quantities are similarly defined for the EME. Using this short-hand, we have

$$
\mathbb{E}\left[d r_{t}^{A a}\right]-d r_{t}^{F} \leq \gamma_{A} \varsigma_{A a, t}+\left(\gamma_{A}-1\right) \varsigma_{\xi A, t}
$$

To summarize, agents in the AE consume at rate $\rho$ per unit of wealth, and they price risky capital according to

$$
\begin{aligned}
& \mathbb{E}\left[d r_{t}^{A a}\right]-d r_{t}^{F}=\gamma_{A} \varsigma_{A a, t}+\left(\gamma_{A}-1\right) \varsigma_{\xi A, t}, \\
& \mathbb{E}\left[d r_{t}^{A b}\right]-d r_{t}^{F} \leq \gamma_{A} \varsigma_{A a, t}+\left(\gamma_{A}-1\right) \varsigma_{\xi A, t} \\
& \mathbb{E}\left[d r_{t}^{E b}\right]-d r_{t}^{F} \leq \gamma_{A} \varsigma_{A e, t}+\left(\gamma_{A}-1\right) \varsigma_{\xi E, t},
\end{aligned}
$$

where

$$
\begin{aligned}
\varsigma_{A a, t} & =\left(x_{A a, t}+x_{A b, t}\right)\left[\left(\sigma+\sigma_{q A, t}\right)^{2}+\sigma_{q E, t}^{2}\right] \\
\varsigma_{\xi A, t} & =\left(\sigma+\sigma_{q A, t}\right) \sigma_{\xi A, t}+\sigma_{q E, t} \sigma_{\xi E, t}
\end{aligned}
$$

\section{A.2 Proof of Proposition 1: Law of Motion for $\eta$}

We conjecture that all equilibrium variables depend endogenously on the share of global wealth held by the AE:

$$
\eta_{t} \equiv \frac{N_{A, t}}{q_{t} K_{t}}
$$

In the subsequent sections, we verify this conjecture, but first we derive the equilibrium law of motion for $\eta_{t}$. Aggregating (12) over all agents in the AE and substituting equations (20), (21), 
and 22, obtains

$$
\begin{aligned}
\frac{d N_{A, t}}{N_{A, t}}= & d r_{t}+\left\{\gamma _ { A } \left[\left(x_{A a, t}+x_{A b, t}\right) \varsigma_{A a, t}+\left(\gamma_{A}-1\right)\left[\left(x_{A a, t}+x_{A b, t}\right) \varsigma_{\xi A, t}-\frac{C_{A, t}}{N_{A, t}}\right\} d t\right.\right. \\
& +\left(x_{A a, t}+x_{A b, t}\right) \Sigma_{A a, t} \cdot d \mathbf{W}_{t} .
\end{aligned}
$$

By Ito's product rule, global wealth evolves according to

$$
\frac{d\left(q_{t} K_{t}\right)}{q_{t} K_{t}}=\left(\mu_{q, t}+\Phi\left(\imath_{t}\right)-\delta+\Sigma_{q, t} \cdot \Sigma_{K, t}\right) d t+\left(\Sigma_{q, t}+\Sigma_{K, t}\right) \cdot d \mathbf{W}_{t}
$$

Expanding the returns equation in 20) allows us to write

$$
\mu_{q, t}+\Phi\left(\imath_{t}\right)-\delta=d r_{t}+\gamma_{A} \varsigma_{A a, t}+\left(\gamma_{A}-1\right) \varsigma_{\xi A, t}-\frac{\bar{a} P_{a, t}-\imath_{t}}{q_{t}}-\sigma \sigma_{q A, t} .
$$

Substituting this and applying Ito's quotient rule yields

$$
\begin{aligned}
\frac{d\left(1 /\left(q_{t} K_{t}\right)\right)}{1 /\left(q_{t} K_{t}\right)}= & \left(\left\|\Sigma_{q, t}+\Sigma_{K, t}\right\|^{2}-d r_{t}-\gamma_{A} \varsigma_{A a, t}-\left(\gamma_{A}-1\right) \varsigma_{\xi A, t}+\frac{\bar{a} P_{a, t}-\imath_{t}}{q_{t}}+\sigma \sigma_{q A, t}-\Sigma_{q, t} \cdot \Sigma_{K, t}\right) d t \\
& -\left(\Sigma_{q, t}+\Sigma_{K, t}\right) \cdot d \mathbf{W}_{t}
\end{aligned}
$$

By Ito's product rule,

$$
\begin{aligned}
\frac{d \eta_{t}}{\eta_{t}}= & \left\{\gamma_{A}\left[\left(x_{A a, t}+x_{A b, t}\right) \varsigma_{A a, t}-\varsigma_{A a, t}\right]+\left(\gamma_{A}-1\right)\left[\left(x_{A a, t}+x_{A b, t}\right) \varsigma_{\xi A, t}-\varsigma_{\xi A, t}\right]-\frac{C_{A, t}}{N_{A, t}}-\Sigma_{q, t} \cdot \Sigma_{K, t}\right\} d t \\
& +\left\{\left\|\Sigma_{q, t}+\Sigma_{K, t}\right\|^{2}+\frac{\bar{a} P_{a, t}-\boldsymbol{l}_{t}}{q_{t}}+\sigma \sigma_{q A, t}-\left(x_{A a, t}+x_{A b, t}\right) \Sigma_{A a, t} \cdot\left(\Sigma_{q, t}+\Sigma_{K, t}\right)\right\} d t \\
& +\left\{\left(x_{A a, t}+x_{A b, t}\right) \Sigma_{A a, t}-\Sigma_{q, t}-\Sigma_{K, t}\right\} \cdot d \mathbf{W}_{t} .
\end{aligned}
$$


This simplifies to

$$
\begin{aligned}
\frac{d \eta_{t}}{\eta_{t}}= & \left\{\frac{\bar{a} P_{a, t}-\imath_{t}}{q_{t}}-\frac{C_{A, t}}{N_{A, t}}+\left(x_{A a, t}+x_{A b, t}-1\right)\left(\gamma_{A} \varsigma_{A a, t}+\left(\gamma_{A}-1\right) \varsigma_{\xi A, t}\right)\right\} d t \\
& +\left\{\left\|\Sigma_{q, t}+\Sigma_{K, t}\right\|^{2}+\sigma \sigma_{q A, t}-\sigma \sigma_{q A, t}-\sigma \sigma_{q E, t}-\left(x_{A a, t}+x_{A b, t}\right) \Sigma_{A a, t} \cdot\left(\Sigma_{q, t}+\Sigma_{K, t}\right)\right\} d t \\
& +\left\{\left(x_{A a, t}+x_{A b, t}\right) \Sigma_{A a, t}-\Sigma_{q, t}-\Sigma_{K, t}\right\} \cdot d \mathbf{W}_{t}
\end{aligned}
$$

We can replace portfolio shares and dot products by observing

$$
\begin{aligned}
x_{A a, t}+x_{A b, t} & =\frac{\psi_{A, t} q_{t} K_{t}}{N_{A, t}}=\frac{\psi_{A, t}}{\eta_{t}} \\
\left\|\Sigma_{q, t}+\Sigma_{K, t}\right\|^{2} & =\left(\psi_{A, t} \sigma+\sigma_{q A, t}\right)^{2}+\left(\psi_{E, t} \sigma+\sigma_{q E, t}\right)^{2} .
\end{aligned}
$$

We can also simplify the volatility vector to become

$$
\begin{aligned}
\Sigma_{\eta t}= & {\left[\begin{array}{c}
\frac{\psi_{A, t}}{\eta_{t}}\left(\sigma+\sigma_{q A, t}\right)-\sigma_{q A, t}-\psi_{A, t} \sigma \\
\frac{\psi_{A, t}}{\eta_{t}} \sigma_{q E, t}-\sigma_{q E, t}-\psi_{E, t} \sigma
\end{array}\right] } \\
= & {\left[\begin{array}{c}
\frac{1-\eta_{t}}{\eta_{t}} \psi_{A, t} \sigma+\left(\frac{\psi_{A, t}}{\eta_{t}}-1\right) \sigma_{q A, t} \\
-\psi_{E, t} \sigma+\left(\frac{\psi_{A, t}}{\eta_{t}}-1\right) \sigma_{q E, t}
\end{array}\right] }
\end{aligned}
$$

Re-arranging and substitutions yield Proposition 1 .

\section{A.3 Proof of Proposition 1: Asset Pricing Condition and Division of the State Space}

In this section, we drop time subscripts.

Asset Pricing Condition. To obtain the global asset pricing condition, we difference the AE's required return on good $a$ and the EME's required return on good $b$, which yields

$$
\frac{\bar{a}\left(P_{a}-P_{b}\right)}{q}+\sigma \sigma_{q A}-\sigma \sigma_{q E}=\gamma_{A} \varsigma_{A a}+\left(\gamma_{A}-1\right) \varsigma_{\xi_{A}}-\gamma_{E} \varsigma_{E b}-\left(\gamma_{E}-1\right) \varsigma_{\zeta E}
$$


The AE Does Not Specialize. In this case, the AE chooses to produce good $b$. Then (21) binds with equality, yielding the equality

$$
\frac{P_{a}}{P_{b}}=\frac{a}{\bar{a}}
$$

which leads to the equations

$$
\begin{aligned}
\psi_{E b} & =\frac{1-\left(1+\frac{1-\alpha}{\alpha}\right) \psi_{A b}}{1+\frac{a}{a} \frac{1-\alpha}{\alpha}} \\
\psi_{A b} & =\frac{1-\left(1+\frac{\bar{a}}{a} \frac{1-\alpha}{\alpha}\right) \psi_{E b}}{1+\frac{1-\alpha}{\alpha}} .
\end{aligned}
$$

Depending on how the algorithm is coded, one of these forms may be more convenient. To pin down $\psi_{A a}$ or $\psi_{E a}$, we use the market clearing condition for consumption, which reduces to

$$
\rho q=\left(\bar{a} \psi_{A a}+\underline{a} \psi_{E a}\right)^{\alpha}\left(\underline{a} \psi_{A b}+\bar{a} \psi_{E b}\right)^{1-\alpha}-\imath(q),
$$

where $l(\cdot)$ is defined as a function of $q$ by Tobin's $q(9)$. Note that this condition holds even if leverage constraints bind

The EME Does Not Specialize. In this case, the EME chooses to produce good $a$. Then we have the equality

$$
\frac{P_{a}}{P_{b}}=\frac{\bar{a}}{\underline{a}},
$$

which leads to the equations

$$
\begin{aligned}
\psi_{A a} & =\frac{1-\left(1+\frac{1-\alpha}{\alpha}\right) \psi_{E a}}{1+\frac{\bar{a}}{\underline{a}} \frac{1-\alpha}{\alpha}} \\
\psi_{E a} & =\frac{1-\left(1+\frac{\bar{a}}{\underline{a}} \frac{1-\alpha}{\alpha}\right) \psi_{A a}}{1+\frac{1-\alpha}{\alpha}} .
\end{aligned}
$$


Depending on how the algorithm is coded, one of these forms may be more convenient. Note that this condition holds even if leverage constraints bind.

Only One Country Does Not Specialize. The previous two sections show that the price ratio of $\operatorname{good} a$ to $\operatorname{good} b$ satisfies

$$
\frac{\underline{a}}{\bar{a}} \leq \frac{P_{a}}{P_{b}} \leq \frac{\bar{a}}{\underline{a}}
$$

In order for the $\mathrm{AE}$ to not specialize, the left inequality must bind with equality, and in order for the EME to not specialize, the right inequality must bind with inequality. Since $\bar{a}>\underline{a}$, only one inequality can bind at a time. Therefore, there are three regions of capital allocations.

1. The AE specializes, the EME does not specialize.

2. Both countries specialize.

3. The AE does not specialize, the EME specializes.

\section{A.4 Proof of Proposition 2}

Suppose that the leverage constraint binds for the AE agents. Then (20) will not bind with equality. However, if the AE agents are levered, then the EME agents are not levered, so their asset-pricing 
condition must still hold. Writing out returns for the EME, we have

$$
\begin{aligned}
\mathbb{E}\left[d r_{t}^{E a}\right]-d r_{t} & \leq \gamma_{E} \varsigma_{E b, t}+\left(\gamma_{E}-1\right) \varsigma_{\zeta E, t} \\
\mathbb{E}\left[d r_{t}^{E b}\right]-d r_{t} & =\gamma_{E} \varsigma_{E b, t}+\left(\gamma_{E}-1\right) \varsigma_{\zeta E, t} \\
\mathbb{E}\left[d r_{t}^{A a}\right]-d r_{t} & \leq \gamma_{E} \varsigma_{E e, t}+\left(\gamma_{E}-1\right) \zeta_{\zeta A, t} \\
\varsigma_{E b, t} & =\frac{\psi_{E, t}}{1-\eta_{t}}\left[\sigma_{q A, t}^{2}+\left(\sigma+\sigma_{q E, t}\right)^{2}\right] \\
\varsigma_{E e, t} & \left.=\frac{\psi_{E, t}}{1-\eta_{t}}\left[\left(\sigma+\sigma_{q A, t}\right) \sigma_{q A, t}+\left(\sigma+\sigma_{q E, t}\right) \sigma_{q E, t}\right]+\sigma_{q E, t}^{2}\right] \\
\varsigma_{\zeta E, t} & =\sigma_{q A, t} \sigma_{\zeta A, t}+\left(\sigma+\sigma_{q E, t}\right) \sigma_{\zeta E, t} \\
\varsigma_{\zeta A, t} & =\left(\sigma+\sigma_{q A, t}\right) \sigma_{\zeta A, t}+\sigma_{q E, t} \sigma_{\zeta E, t} .
\end{aligned}
$$

Expanding the excess returns for production of intermediate goods yields

$$
\begin{aligned}
& \mathbb{E}\left[d r_{t}^{A a}\right]=\frac{\bar{a} P_{a, t}-\imath_{t}}{q_{t}}+\mu_{q, t}+\Phi\left(l_{t}\right)-\delta+\sigma \sigma_{q A, t}, \\
& \mathbb{E}\left[d r_{t}^{E b}\right]=\frac{\bar{a} P_{b, t}-\imath_{t}}{q_{t}}+\mu_{q, t}+\Phi\left(l_{t}\right)-\delta+\sigma \sigma_{q E, t}
\end{aligned}
$$

Observe the similar terms in these two expressions. By adding and subtracting the appropriate terms, we can write the excess returns from producing good $a$ for the AE in terms of the excess returns from producing good $b$ for the EME:

$$
\mathbb{E}\left[d r_{t}^{A a}\right]-d r_{t}=\mathbb{E}\left[d r_{t}^{E b}\right]-d r_{t}+\frac{\bar{a}\left(P_{a, t}-P_{b, t}\right)}{q_{t}}+\sigma \sigma_{q A, t}-\sigma \sigma_{q E, t}
$$

Since the EME's asset-pricing condition still holds when the AE is constrained, we have

$$
\mathbb{E}\left[d r_{t}^{A a}\right]-d r_{t}=\gamma_{E} \varsigma_{E b, t}+\left(\gamma_{E}-1\right) \zeta_{\zeta E, t}+\frac{\bar{a}\left(P_{a, t}-P_{b, t}\right)}{q_{t}}+\sigma \sigma_{q A, t}-\sigma \sigma_{q E, t},
$$

where we continue to use the notation that $\varsigma_{i j, t}$ denotes the risk premium required by Country $i$ when investing in returns on good $j$ or equity as if their risk aversion was one. We may similarly 
write the excess returns for the other expressions:

$$
\begin{aligned}
& \mathbb{E}\left[d r_{t}^{A b}\right]-d r_{t} \leq \gamma_{E} \varsigma_{E b, t}+\left(\gamma_{E}-1\right) \zeta_{\zeta E, t}-\frac{(\bar{a}-\underline{a}) P_{b, t}}{q_{t}}+\sigma \sigma_{q A, t}-\sigma \sigma_{q E, t}, \\
& \mathbb{E}\left[d r_{t}^{E b}\right]-d r_{t}=\gamma_{E} \varsigma_{E b, t}+\left(\gamma_{E}-1\right) \zeta_{\zeta E, t} .
\end{aligned}
$$

We can repeat this same exercise for the EME. If leverage constraints bind for $E$, then excess returns satisfy

$$
\begin{aligned}
& \mathbb{E}\left[d r_{t}^{E a}\right]-d r_{t} \leq \gamma_{A} \varsigma_{A a, t}+\left(\gamma_{A}-1\right) \varsigma_{\xi A, t}-\frac{(\bar{a}-\underline{a}) P_{a, t}}{q_{t}}-\sigma \sigma_{q A, t}+\sigma \sigma_{q E, t}, \\
& \mathbb{E}\left[d r_{t}^{E b}\right]-d r_{t}=\gamma_{A} \varsigma_{A a, t}+\left(\gamma_{A}-1\right) \varsigma_{\xi A, t}-\frac{\bar{a}\left(P_{a, t}-P_{b, t}\right)}{q_{t}}-\sigma \sigma_{q A, t}+\sigma \sigma_{q E, t}, \\
& \mathbb{E}\left[d r_{t}^{A a}\right]-d r_{t} \leq \gamma_{A} \varsigma_{A a, t}+\left(\gamma_{A}-1\right) \varsigma_{\xi A, t} .
\end{aligned}
$$

To derive the appropriate law of motion, observe that the AE's asset-pricing conditions always hold true as long as it is not constrained. Because we only used the AE's conditions in the original derivation of $\eta_{t}$ without leverage constraints, when the EME is constrained, the same law of motion still applies. The difference will be the set of equations that pin down capital allocations.

However, if constraints bind for the AE, then our stated law of motion is incorrect because, for example,

$$
\mathbb{E}\left[d r_{t}^{A a}\right]-d r_{t} \neq \gamma_{A} \varsigma_{A a, t}+\left(\gamma_{A}-1\right) \varsigma_{\xi A, t}
$$

To rectify this, we re-write

$$
\begin{aligned}
\frac{d N_{A t}}{N_{A t}}= & d r_{t}-\frac{c_{A t}}{n_{A t}} d t+\frac{\psi_{A a, t}}{\eta_{t}}\left(\gamma_{E} \varsigma_{E b, t}+\left(\gamma_{E}-1\right) \varsigma_{\zeta E, t}+\frac{\bar{a}\left(P_{a, t}-P_{b, t}\right)}{q_{t}}+\sigma \sigma_{q A, t}-\sigma \sigma_{q E, t}\right) d t \\
& +\frac{\psi_{A b, t}}{\eta_{t}}\left(\gamma_{E} \varsigma_{E b, t}+\left(\gamma_{E}-1\right) \zeta_{\zeta E, t}-\frac{\left.(\bar{a}-\underline{a}) P_{b, t}\right)}{q_{t}}+\sigma \sigma_{q A, t}-\sigma \sigma_{q E, t}\right) d t \\
& \left.+\left(\gamma_{E}-1\right) \varsigma_{\zeta E, t}\right) d t+\frac{\psi_{A, t}}{\eta_{t}} \Sigma_{A a, t} \cdot d \mathbf{W}_{t} .
\end{aligned}
$$


Adjusting the law of motion for aggregate wealth yields

$$
\begin{aligned}
\frac{d\left(1 /\left(q_{t} K_{t}\right)\right.}{1 /\left(q_{t} K_{t}\right)}= & \left(\left\|\Sigma_{q, t}+\Sigma_{K, t}\right\|^{2}-d r_{t}-\gamma_{E} \varsigma_{E b, t}-\left(\gamma_{E}-1\right) \zeta_{\zeta E, t}+\frac{\bar{a} P_{b, t}-\imath_{t}}{q_{t}}+\sigma \sigma_{q E, t}-\Sigma_{q, t} \cdot \Sigma_{K, t}\right) d t \\
& -\left(\Sigma_{q, t}+\Sigma_{K, t}\right) \cdot d \mathbf{W}_{t}
\end{aligned}
$$

By Ito's product rule,

$$
\begin{aligned}
\frac{d \eta_{t}}{\eta_{t}}= & -\frac{c_{A t}}{n_{A t}} d t+\frac{\psi_{A a, t}}{\eta_{t}}\left(\gamma_{E} \varsigma_{E b, t}+\left(\gamma_{E}-1\right) \varsigma_{\zeta E, t}+\frac{\bar{a}\left(P_{a, t}-P_{b, t}\right)}{q_{t}}+\sigma \sigma_{q A, t}-\sigma \sigma_{q E, t}\right) d t \\
& \left.+\frac{\psi_{A b, t}}{\eta_{t}}\left(\gamma_{E} \varsigma_{E b, t}+\left(\gamma_{E}-1\right) \zeta_{\zeta E, t}-\frac{\left.(\bar{a}-\underline{a}) P_{b, t}\right)}{q_{t}}+\sigma \sigma_{q A, t}-\sigma \sigma_{q E, t}+\left(\gamma_{E}-1\right) \varsigma_{\zeta E, t}\right)\right) d t \\
& +\left(\left\|\Sigma_{q, t}+\Sigma_{K, t}\right\|^{2}-\gamma_{E} \varsigma_{E b, t}-\left(\gamma_{E}-1\right) \varsigma_{\zeta E, t}+\frac{\bar{a} P_{b, t}-\boldsymbol{l}_{t}}{q_{t}}+\sigma \sigma_{q E, t}-\Sigma_{q, t} \cdot \Sigma_{K, t}\right) d t \\
& -\frac{\psi_{A, t}}{\eta_{t}} \Sigma_{A a, t} \cdot\left(\Sigma_{q, t}+\Sigma_{K, t}\right) d t+\left[\frac{\psi_{A, t}}{\eta_{t}} \Sigma_{A a, t}-\Sigma_{q, t}-\Sigma_{K, t}\right] \cdot d \mathbf{W}_{t} .
\end{aligned}
$$

Focusing on the drift, we have the simplification

$$
\begin{aligned}
\mu_{\eta t}= & \left.\left(\frac{\psi_{A, t}}{\eta_{t}}-1\right)\left(\gamma_{E} \varsigma_{E b, t}+\left(\gamma_{E}-1\right) \varsigma_{\zeta E, t}+\sigma \sigma_{q A, t}-\sigma \sigma_{q E, t}\right)+\left(\gamma_{E}-1\right) \varsigma_{\zeta E, t}\right) \\
& +\frac{\psi_{A a, t}}{\eta_{t}} \frac{\bar{a} P_{a, t}}{q_{t}}-\frac{\psi_{A, t}}{\eta_{t}} \frac{\bar{a} P_{b, t}}{q_{t}}+\frac{\psi_{A b, t}}{\eta_{t}} \frac{a P_{b, t}}{q_{t}}-\frac{C_{A, t}}{N_{A, t}}+\frac{\bar{a} P_{b, t}-\imath_{t}}{q_{t}} \\
& +\sigma \sigma_{q E, t}-\psi_{A, t} \sigma \sigma_{q A, t}-\psi_{E, t} \sigma \sigma_{q E, t} \\
& +\left\|\Sigma_{q, t}+\Sigma_{K, t}\right\|^{2}-\frac{\psi_{A, t}}{\eta_{t}} \Sigma_{A a, t} \cdot\left(\Sigma_{q, t}+\Sigma_{K, t}\right) \\
= & \left.\left(\frac{\psi_{A, t}}{\eta_{t}}-1\right)\left(\gamma_{E} \varsigma_{E b, t}+\left(\gamma_{E}-1\right) \varsigma_{\zeta E, t}-\frac{\bar{a} P_{b, t}}{q_{t}}\right)-\frac{l_{t}}{q_{t}}+\left(\gamma_{E}-1\right) \zeta_{\zeta E, t}\right) \\
& +\frac{\psi_{A a, t}}{\eta_{t}} \frac{\bar{a} P_{a, t}}{q_{t}}+\frac{\psi_{A b, t}}{\eta_{t}} \frac{a P_{b, t}}{q_{t}}-\frac{C_{A, t}}{N_{A, t}}+\left[\left(\frac{\psi_{A, t}}{\eta_{t}}-1\right)-\psi_{A, t}\right]\left(\sigma \sigma_{q A, t}-\sigma \sigma_{q E, t}\right) \\
& +\left\|\Sigma_{q, t}+\Sigma_{K, t}\right\|^{2}-\frac{\psi_{A, t}}{\eta_{t}} \Sigma_{A a, t} \cdot\left(\Sigma_{q, t}+\Sigma_{K, t}\right)
\end{aligned}
$$

Simplification yields Proposition 2. 


\section{A.5 Equilibrium ODEs}

To solve for equilibrium, we construct a system of ODEs in three dependent variables: $q$, $\xi$, and $\zeta$. It is convenient to work with a change of variables for $\xi$ and $\zeta$ because it allows us to reduce the system to a first-order differential equation in $q$ and two other second order ODEs. If we directly constructed ODEs for $\xi$ and $\zeta$ from the HJBs of Countries the AE and EME, then we would need to solve a system of three second-order differential equations, as in Di Tella (2017).

Change of Variables. Define $v_{A}$ and $v_{E}$ by

$$
v_{A}=\xi \eta q, \quad v_{E}=\zeta(1-\eta) q
$$

Then

$$
\begin{aligned}
V_{A} & =\frac{(\xi \eta q K)^{1-\gamma_{A}}}{1-\gamma_{A}}=\frac{\left(v_{A} K\right)^{1-\gamma_{A}}}{1-\gamma_{A}} \\
V_{E} & =\frac{(\zeta(1-\eta) q K)^{1-\gamma_{E}}}{1-\gamma_{E}}=\frac{\left(v_{E} K\right)^{1-\gamma_{E}}}{1-\gamma_{E}}
\end{aligned}
$$

We can therefore write

$$
\begin{aligned}
& \mathbb{E}\left[d V_{A}\right]=\left(v_{A} K\right)^{1-\gamma_{A}}\left(\mu_{v A}+\Phi(\boldsymbol{\imath})-\delta+\Sigma_{v A} \cdot \Sigma_{K}\right)-\frac{\gamma_{A}}{2}(v K)^{1-\gamma_{A}}\left\|\Sigma_{v A}+\Sigma_{K}\right\|^{2} \\
& \mathbb{E}\left[d V_{E}\right]=\left(v_{E} K\right)^{1-\gamma_{E}}\left(\mu_{v E}+\Phi(\imath)-\delta+\Sigma_{v E} \cdot \Sigma_{K}\right)-\frac{\gamma_{E}}{2}(v K)^{1-\gamma_{E}}\left\|\Sigma_{v E}+\Sigma_{K}\right\|^{2}
\end{aligned}
$$

Plugging back into the HJBs for the AE and EME yields

$$
\begin{aligned}
0= & \rho\left(v_{A} K\right)^{1-\gamma_{A}}\left(\log (\rho \eta q K)-\log \left(v_{A} K\right)\right) \\
& +\left(v_{A} K\right)^{1-\gamma_{A}}\left(\mu_{v A}+\Phi(\boldsymbol{\imath})-\delta+\Sigma_{v_{A}} \cdot \Sigma_{K}\right)-\frac{\gamma_{A}}{2}\left(v_{A} K\right)^{1-\gamma_{A}}\left\|\Sigma_{v_{A}}+\Sigma_{K}\right\|^{2} \\
\mu_{v A}= & \frac{\gamma_{A}}{2}\left[\left(\psi_{A} \sigma_{A}+\sigma_{v_{A} A}\right)^{2}+\left(\psi_{E} \sigma_{E}+\sigma_{v_{A} E}\right)^{2}\right] \\
& +\rho\left(\log \left(v_{A}\right)-\log (\rho q \eta)\right)-(\Phi(\boldsymbol{l})-\delta)-\psi_{A} \sigma_{A} \sigma_{v_{A} A}-\psi_{E} \sigma_{E} \sigma_{v_{A} E}
\end{aligned}
$$


and

$$
\begin{aligned}
\mu_{v E}= & \frac{\gamma_{E}}{2}\left[\left(\psi_{A} \sigma_{A}+\sigma_{v_{E} A}\right)^{2}+\left(\psi_{E} \sigma_{E}+\sigma_{v_{E} E}\right)^{2}\right] \\
& +\rho\left(\log \left(v_{E}\right)-\log (\rho q(1-\eta))\right)-(\Phi(\imath)-\delta)-\psi_{A} \sigma_{A} \sigma_{v_{E} A}-\psi_{E} \sigma_{E} \sigma_{v_{E} E} .
\end{aligned}
$$

By Ito’s lemma, we also have

$$
\begin{aligned}
& \mu_{v A}=\frac{\partial_{\eta} v_{A}}{v_{A}} \mu_{\eta} \eta+\frac{1}{2} \frac{\partial_{\eta \eta} v_{A}}{v_{A}} \eta^{2}\left\|\Sigma_{\eta}\right\|^{2} \\
& \mu_{v E}=\frac{\partial_{\eta} v_{E}}{v_{E}} \mu_{\eta} \eta+\frac{1}{2} \frac{\partial_{\eta \eta} v_{E}}{v_{E}} \eta^{2}\left\|\Sigma_{\eta}\right\|^{2}
\end{aligned}
$$

Equating these two expressions for the drifts of $v_{A}$ and $v_{E}$, respectively, yields two second-order differential equations for $v_{A}$ and $v_{E}$.

This formulation of the HJB is particularly convenient since we have the equalities

$$
\begin{aligned}
& \sigma_{v_{A} A}=\sigma_{\xi_{A}}+\sigma_{\eta A}+\sigma_{q A}, \\
& \sigma_{v_{A} E}=\sigma_{\xi E}+\sigma_{\eta E}+\sigma_{q E},
\end{aligned}
$$

hence we may write

$$
\begin{gathered}
\sigma_{\xi_{A}}=\sigma_{v_{A} A}-\left(\sigma_{\eta A}+\sigma_{q A}\right) \\
\sigma_{\xi_{E}}=\sigma_{v_{A} E}-\left(\sigma_{\eta E}+\sigma_{q E}\right) .
\end{gathered}
$$

For $v_{E}$, observe that by Ito's lemma,

$$
\frac{d(1-\eta)}{1-\eta}=-\frac{1}{1-\eta} d \eta=-\frac{\eta}{1-\eta} \mu_{\eta} d t-\frac{\eta}{1-\eta} \Sigma_{\eta} \cdot d \mathbf{W}_{t}
$$


hence

$$
\begin{aligned}
& \sigma_{\zeta A}=\sigma_{v_{E} A}+\frac{\eta}{1-\eta} \sigma_{\eta A}-\sigma_{q A} \\
& \sigma_{\zeta E}=\sigma_{v_{E} E}+\frac{\eta}{1-\eta} \sigma_{\eta E}-\sigma_{q E} .
\end{aligned}
$$

ODE System. The first-order ODE pinning down $q$ is equation (27), which is an implicit firstorder differential equation in $q, \xi$, and $\zeta$. Note that we do not have to actually compute the derivatives of $\xi$ and $\zeta$. Instead, we may use the identities relating the volatilities of $v_{A}$ and $v_{B}$ with $\xi$ and $\zeta$, respectively. All together, we have the following system of ODEs:

$$
\begin{aligned}
\frac{\bar{a}\left(P_{a}-P_{b}\right)}{q}+\sigma \sigma_{q A}-\sigma \sigma_{q E}= & \gamma_{A} \varsigma_{A a}+\left(\gamma_{A}-1\right) \varsigma_{\xi A}-\gamma_{E} \varsigma_{E b}-\left(\gamma_{E}-1\right) \varsigma_{\zeta E} \\
\frac{\partial_{\eta} v_{A}}{v_{A}} \mu_{\eta} \eta+\frac{1}{2} \frac{\partial_{\eta \eta} v_{A}}{v_{A}} \eta^{2}\left\|\Sigma_{\eta}\right\|^{2}= & \frac{\gamma_{A}}{2}\left[\left(\psi_{A} \sigma_{A}+\sigma_{v_{A} A}\right)^{2}+\left(\psi_{E} \sigma_{E}+\sigma_{v_{A} E}\right)^{2}\right] \\
& +\rho\left(\log \left(v_{A}\right)-\log (\rho q \eta)\right)-(\Phi(\imath)-\delta) \\
& -\psi_{A} \sigma_{A} \sigma_{v_{A} A}-\psi_{E} \sigma_{E} \sigma_{v_{A} E} \\
\frac{\partial_{\eta} v_{E}}{v_{E}} \mu_{\eta} \eta+\frac{1}{2} \frac{\partial_{\eta \eta} v_{E}}{v_{E}} \eta^{2}\left\|\Sigma_{\eta}\right\|^{2}= & \frac{\gamma_{E}}{2}\left[\left(\psi_{A} \sigma_{A}+\sigma_{v_{E} A}\right)^{2}+\left(\psi_{E} \sigma_{E}+\sigma_{v_{E} E}\right)^{2}\right] \\
& +\rho\left(\log \left(v_{E}\right)-\log (\rho q(1-\eta))\right)-(\Phi(\imath)-\delta) \\
& -\psi_{A} \sigma_{A} \sigma_{v_{E} A}-\psi_{E} \sigma_{E} \sigma_{v_{E} E} .
\end{aligned}
$$

\section{A.6 Consumption-Equivalent Welfare Gains and Losses}

We suppress time subscripts here. Because we know the form of agents' value functions, we can sharply quantiy a country's consumption-equivalent welfare gains or losses in differnt equilibria. Let a superscript of (0) denote objects from some initial equilibrium and a superscript of (1) denote objects from an alternative equilibrium. Define $\phi_{g}$ as the percentage gain in consumptionequivalent welfare. Consider the AE. With unit intertemporal elasticity of substitution, $\rho n_{A}=c_{A}$, so a percentage increase in the AE's consumption stream is equivalent to a percentage increase in the AE's net worth. Holding fixed the AE's marginal value of wealth in the initial equilibrium, we 
have that

$$
V_{A}^{(1)}=\frac{\left(\xi^{(0)}\left(1+\phi_{g}\right) c_{A}^{(0)} / \rho\right)^{1-\gamma_{A}}}{1-\gamma_{A}}=\left(1+\phi_{g}\right)^{1-\gamma_{A}} \frac{\left(\xi^{(0)} c_{A}^{(0)} / \rho\right)^{1-\gamma_{A}}}{1-\gamma_{A}}=\left(1+\phi_{g}\right)^{1-\gamma_{A}} V_{A}^{(0)}
$$

or equivalently,

$$
\phi_{g}=\left(\frac{V_{A}^{(1)}}{V_{A}^{(0)}}\right)^{1 /\left(1-\gamma_{A}\right)}-1
$$

For the consumption-equivalent welfare loss, the percentage loss $\phi_{c}$ is given by

$$
\phi_{c}=1-\left(\frac{V_{A}^{(1)}}{V_{A}^{(0)}}\right)^{1 /\left(1-\gamma_{A}\right)} .
$$

\section{B Benchmark Cases}

Here we analyze in more detail the benchmark cases we use to discern the mechanisms underlying our results.

\section{B.1 Endogenous Risk}

Brunnermeier and Sannikov (2014b) highlight the role of endogenous risk from $\Sigma_{q}$ in generating systemic risk. Because $\Sigma_{\eta}$ increases with the level of asset price volatility, endogenous risk affects the long-run stability of the economy. To get a better idea of the role endogenous risk plays in our model, we shut off the terms of trade hedge by assuming there are no intermediate goods. Instead, countries directly produce the final consumption good with a linear production technology as in Brunnermeier and Sannikov (2014b). We assume the AE is more productive with capital than the EME because if both countries are equally productive with capital, then $q$ is constant in equilibrium. Let $\bar{a}$ be the AE's productivity and $\underline{a}$ be the EME's productivity, with $\bar{a}>\underline{a}$.

The model becomes similar to the model in Brunnermeier and Sannikov (2014b) 22 The econ-

\footnotetext{
${ }^{22}$ The primary difference is that we obtain a nondegenerate stationary distribution by implementing redistributive taxes. The AE pays a fraction $v$ of their net worth in taxes to the EME, while the EME pays a fraction $\tau$ of their net
} 
omy is solved in two regions. In the first region, the more productive country does not hold all capital. Countries choose their capital holdings so that holds

$$
\bar{a}-\underline{a}=\gamma_{A} \varsigma_{A a}-\left(\gamma_{A}-1\right) \varsigma_{\xi A}-\gamma_{E} \varsigma_{E b}-\left(\gamma_{E}-1\right) \varsigma_{\zeta E}
$$

in equilibrium. In the second region, $\psi_{A}=1$. By market clearing for consumption, the price of capital is 23

$$
q=\frac{\bar{a} \psi_{A}+\underline{a}\left(1-\psi_{A}\right)+1 / \kappa}{\rho+\lambda / \kappa} .
$$

Figure 9 illustrates equilibrium in this special case. Because the countries are no longer symmetric in productivity, the drift and volatility of $\eta$ do not exhibit the same symmetric shape as the original model. Because countries directly produce the final consumption good rather than intermediate goods, there is no terms of trade hedge present. Despite this, leverage constraints still have a stabilizing effect. Moreover, their impact on equilibrium also resembles the original model's. Figure 9(a) shows that the drift of $\eta$ again starts to peak sooner and at a lower maximum as leverage constraints tighten. Similarly, Figure 9 (b) indicates that lower leverage constraints decrease the volatility of $\eta$. For constraints $L_{A}=1$ and $L_{A}=0.5$, these effects cause the stationary distribution to shift toward the right. Therefore, the terms of trade hedge does not entirely explain the improvement in long-run financial stability.

Recall that $\mu_{\eta}$ also depends on leverage choices, the volatility of asset prices, and the volatility of $\xi$, which is the marginal value of the AE's net worth (see the drift in (16)). Panels (a) and (b) in Figure 10 show that leverage constraints dampen the average volatilities of asset prices and $\xi$ but shift the peak volatility away from $\eta=0$. By altering equilibrium volatility, the AE indirectly affects the returns earned from risk taking and causes the behavior in the drift of $\eta$ shown in worth in taxes to the AE. The former guarantees the AE does not accumulate all wealth in the world econommy, while the latter guarantees the AE's net worth does not get stuck near zero due to the first tax.

${ }^{23}$ When numerically solving for equilibrium, it is more convenient to write $\psi_{A}$ as a function of $q$ :

$$
\psi_{A}=\frac{(\rho \kappa+\lambda) q-1-\kappa \underline{a}}{\kappa(\bar{a}-\underline{a})} .
$$




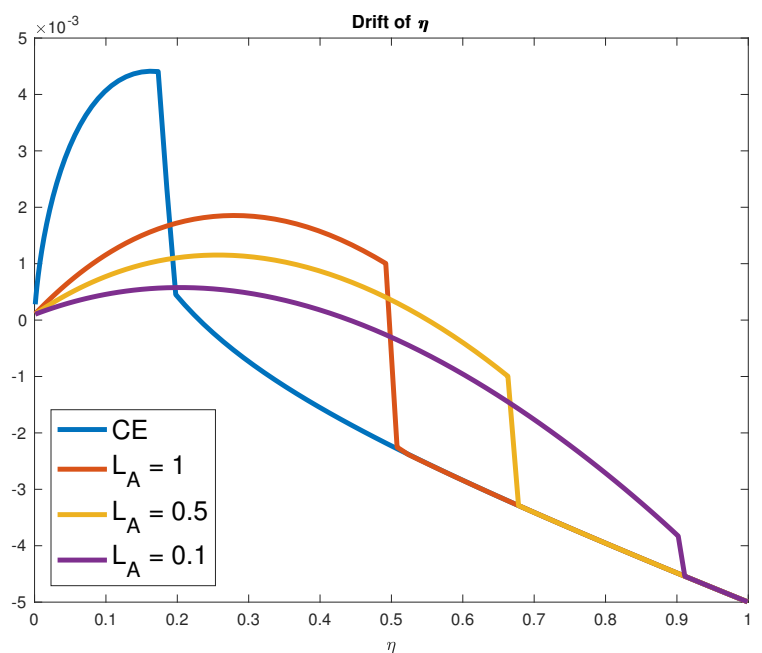

(a) Drift $\mu_{\eta} \eta$

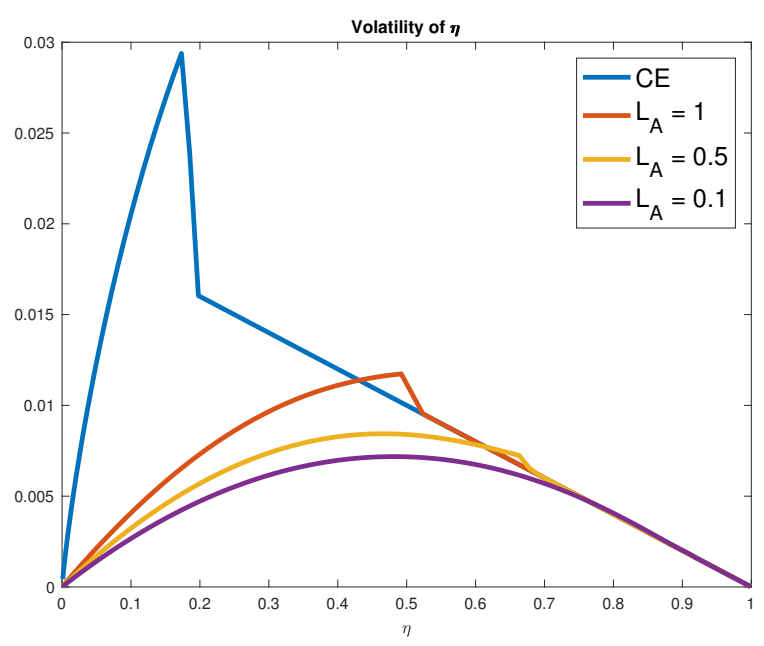

(b) Volatility $\sigma_{\eta} \eta$

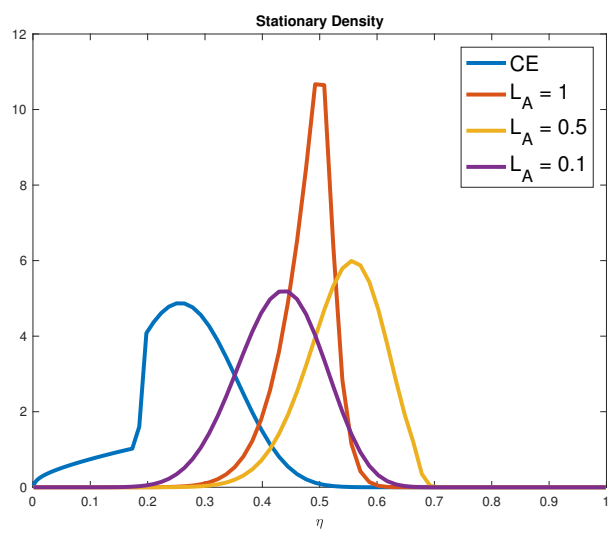

(c) Stationary Density

Figure 9: Panels (a) - (c) illustrate equilibrium with a linear investment technology while varying leverage constraints. Panel (a) plots the terms of trade, Panel (b) plots the drift of $\eta$, Panel (c) plots the volatility of $\eta$, and Panel (d) plots the stationary distributions. In all cases, the EME does not use a leverage constraint. The competitive equilibrium is in blue, $L_{A}=1$ is in red, $L_{A}=0.5$ is in yellow, and $L_{A}=0.1$ is in purple. 
Figure 9 (a). Panels (c) and (d) in Figure 10 confirm that the same changes in volatilities occur in the original model with two intermediate goods. Even without a terms of trade hedge, leverage constraints can still stabilize the stationary distribution by influencing equilibrium volatility.

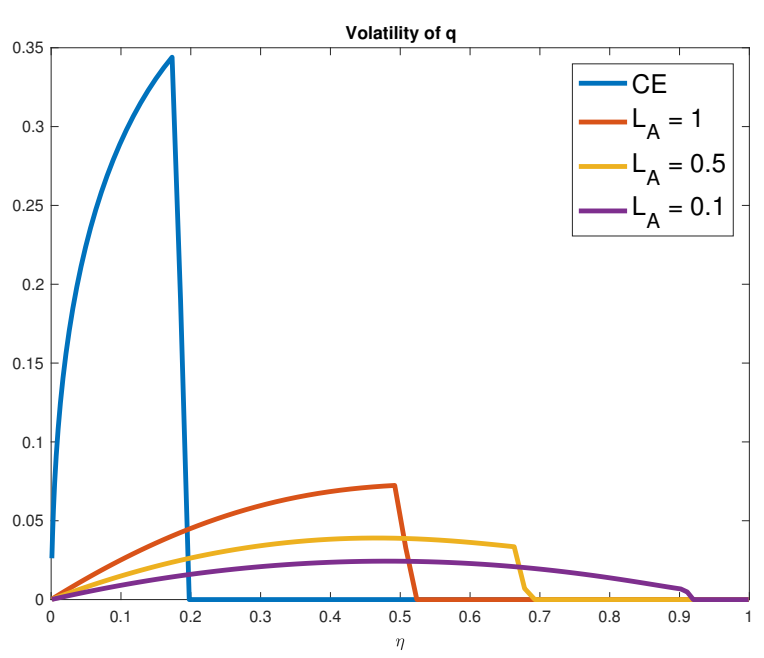

(a) Volatility of $q, 1$ Good

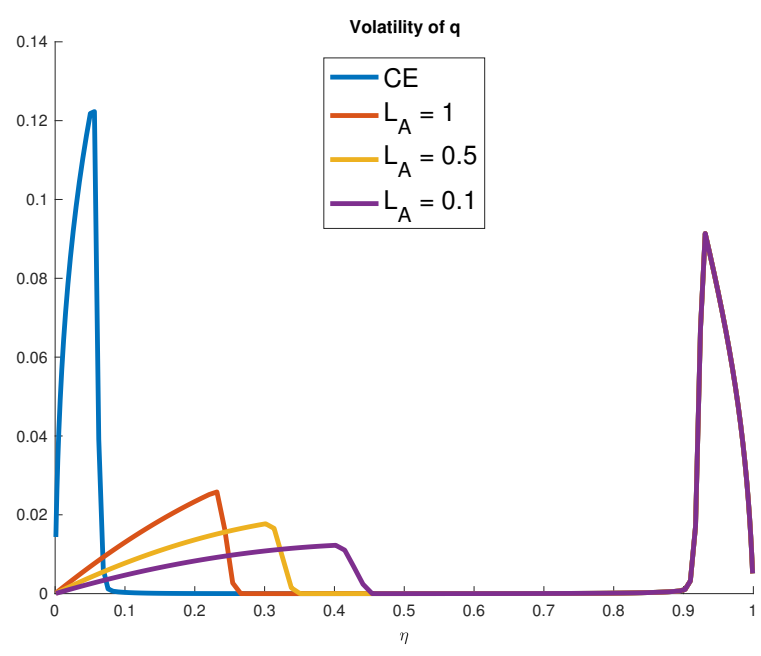

(c) Volatility of $q, 2$ Goods

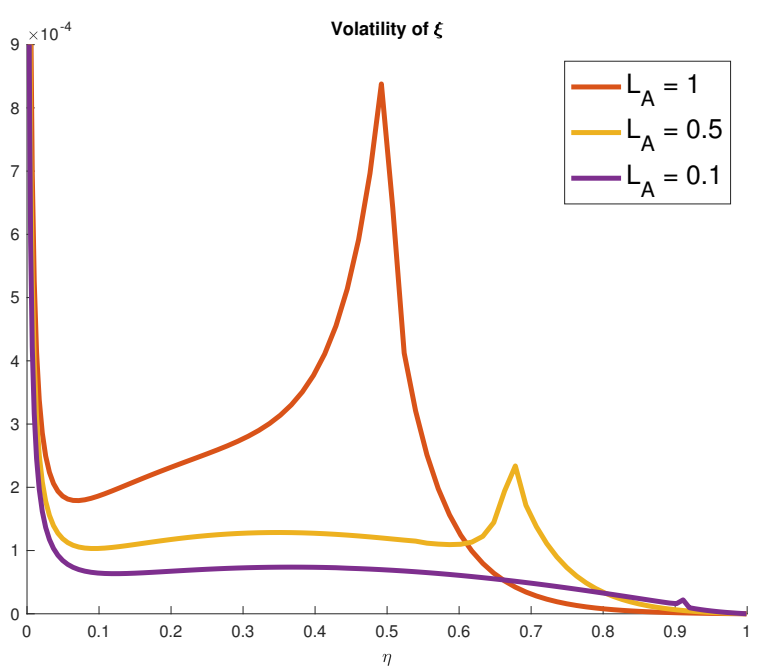

(b) Volatility of $\xi, 1$ Good

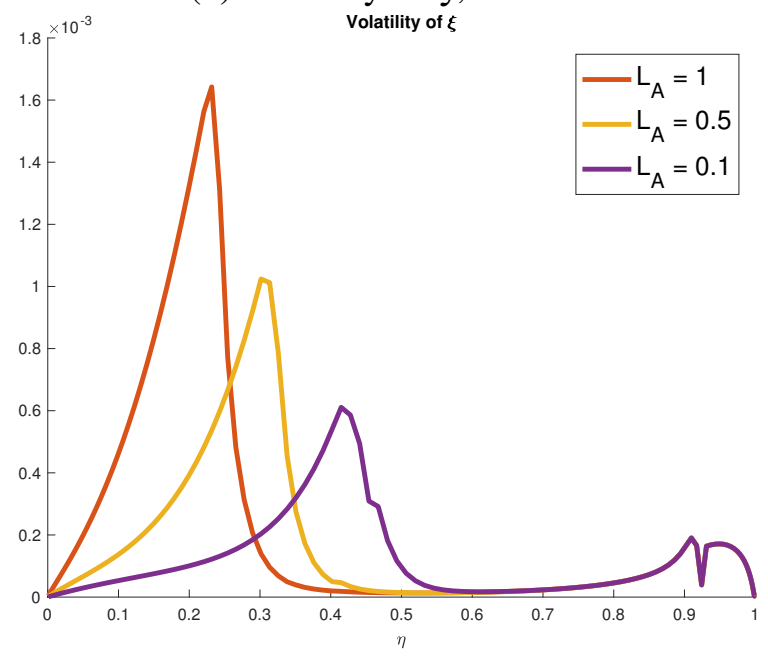

(d) Volatility of $\xi, 2$ Goods

Figure 10: Panels (a) and (b) plot the volatilities of $q$ and $\xi$, respectively, when there is only one good produced by capital. Panels (c) and (d) plot the same volatilities but from the original model. In all cases, the EME does not use a leverage constraint. The competitive equilibrium is in blue, $L_{A}=1$ is in red, $L_{A}=0.5$ is in yellow, and $L_{A}=0.1$ is in purple. 


\section{B.2 Terms of Trade Hedge}

As a counterpart to the previous section's analysis, we now focus on the terms of trade hedge and shut off amplification through asset prices by assuming $\Phi(l)$ is a linear investment technology. Without asset price amplification, endogenous volatility only emerges from countries' choice of leverage. For simplicity, assume $\Phi(\imath)=\lambda \iota$. Tobin's q implies

$$
\Phi^{\prime}(l)=\lambda=\frac{1}{q} \Rightarrow q=\frac{1}{\lambda},
$$

hence the price of capital is constant, so asset prices are not volatile.

Figure 11 demonstrates that equilibrium in this special case closely resembles our original model. Tighter leverage constraints still cause the terms of trade hedge to apply sooner when $\psi_{A}$ deviates from its optimal value, as Panel (a) shows. Panel (b) indicates that this effect on the terms of trade is associated with the drift of $\eta$ peaking faster but at a smaller maximum, just like in the original model. Because leverage constraints restrict the leverage ratio $\psi_{A} / \eta$, the volatility of $\eta$ also decreases. These changes in the drift and volatility of $\eta$ cause the stationary distribution to shift its mass to the right. Although we have shut off asset price amplification, the same phenomena that characterized equilibrium in our original model still appear.

Although the previous and current sections indicate that leverage constraints promote financial stability through both endogenous risk and the terms of trade hedge, the latter mechanism is the stronger factor. In the one good model, tighter leverage constraints do not always generate a stabler stationary distribution. Figure 9 (c) shows that the stationary distribution when $L_{A}=0.1$ is substantially left of the stationary distribution when $L_{A}=0.5$. Tightening $L_{A}=0.5$ to $L_{A}=0.1$ actually decreases long-run stability. In contrast, Figure 11)(d) suggests that tighter constraints always shift the distribution to the right like in the original model. This difference points to the terms of trade hedge as the more important mechanism for why leverage constraints improve outcomes for the AE. 


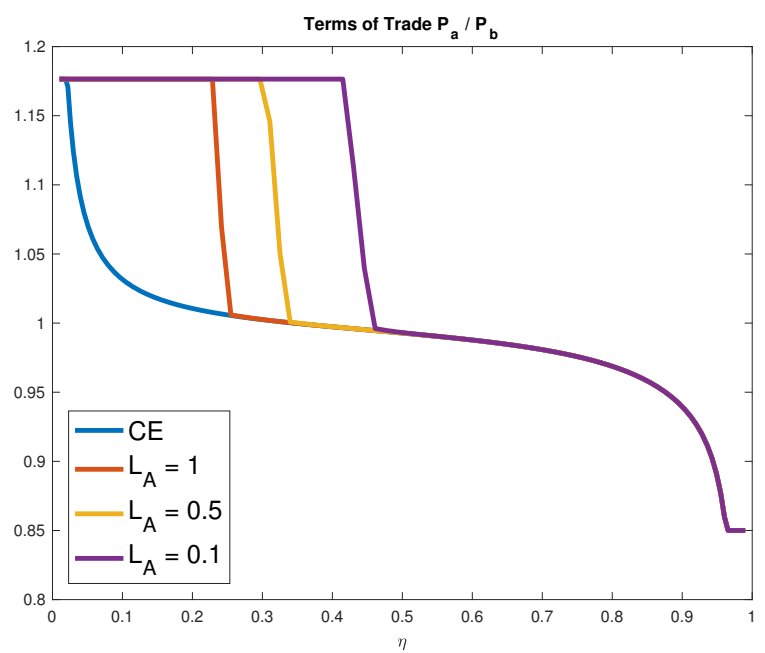

(a) Terms of Trade $P_{a} / P_{b}$

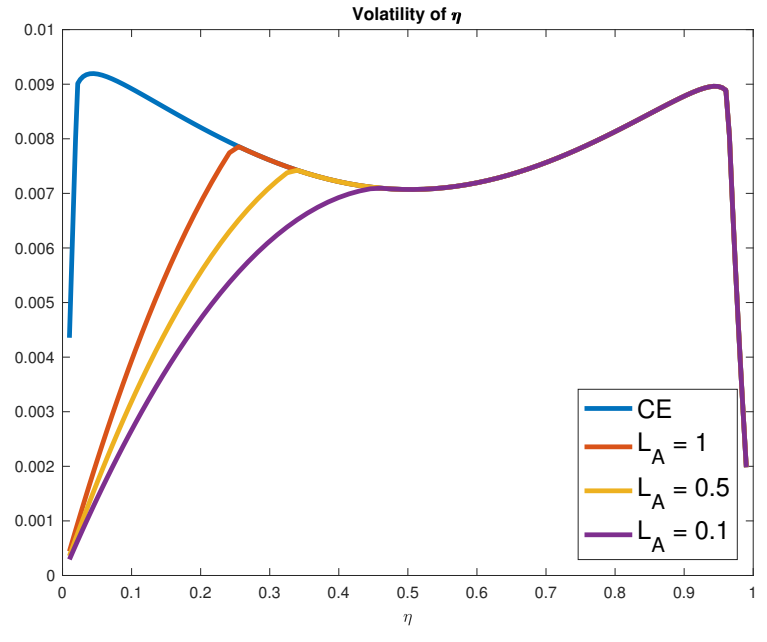

(c) Volatility $\sigma_{\eta} \eta$

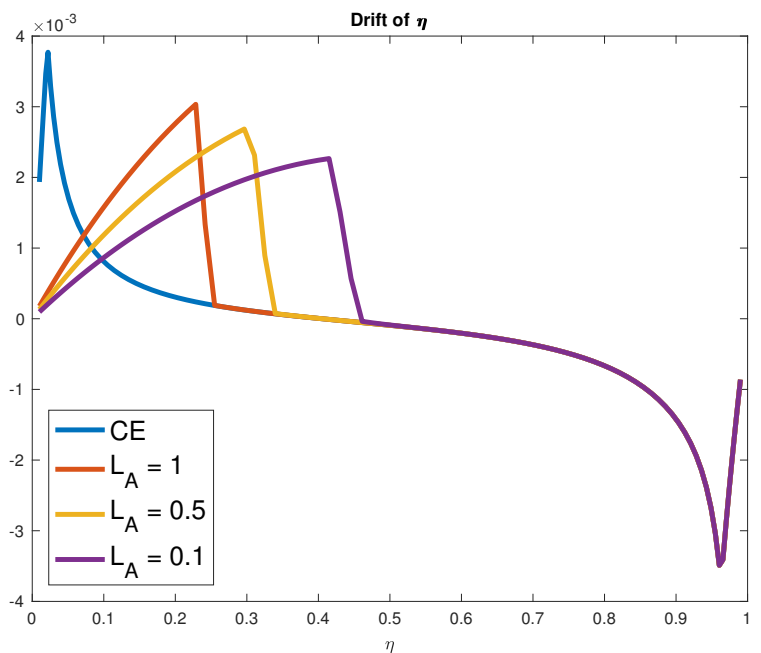

(b) Drift $\mu_{\eta} \eta$

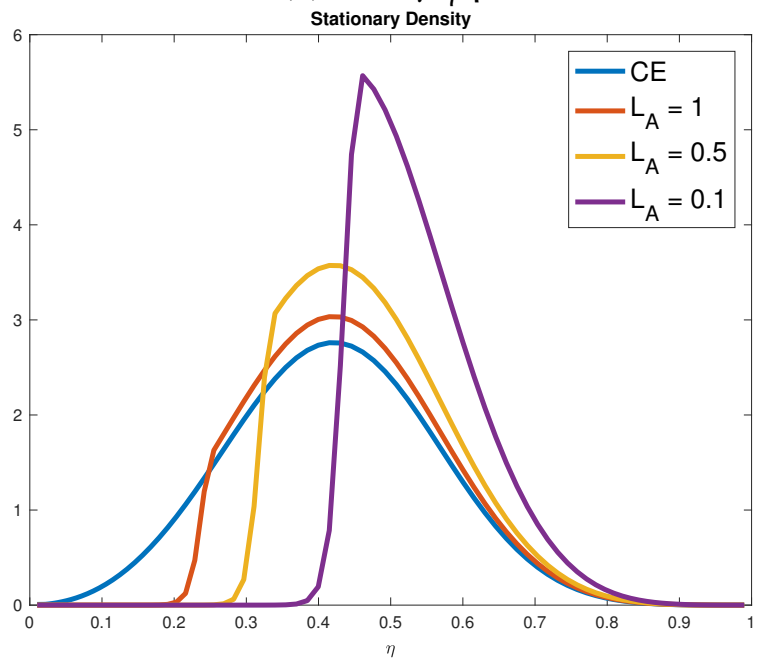

(d) Stationary Density

Figure 11: Panels (a) - (d) illustrate equilibrium with a linear investment technology while varying leverage constraints. Panel (a) plots the terms of trade, Panel (b) plots the drift of $\eta$, Panel (c) plots the volatility of $\eta$, and Panel (d) plots the stationary distributions. In all cases, the EME does not use a leverage constraint. The competitive equilibrium is in blue, $L_{A}=1$ is in red, $L_{A}=0.5$ is in yellow, and $L_{A}=0.1$ is in purple.

\section{B.3 Counterfactual Laws of Motion for $\eta$.}

Here, we examine the relative contributions of equilibrium risk pricing and the terms of trade hedge from another perspective. Figure 12 presents the drifts of $\eta$ and stationary distributions in "counterfactual" scenarios. The solid blue and red lines in Figure 12(a) and (b) are $\mu_{\eta}$ from 
the competitive equilibrium and the $L_{A}=1$ equilibrium. The yellow dashed line in Panel (a) is a "counterfactual" $\mu_{\eta}$ from the competitive equilibrium where we replace any prices of intermediate goods in the expression for $\mu_{\eta}$ in (16) by the prices from the $L_{A}=1$ equilibrium. For example, in the counterfactual drift, the first term $\bar{a} P_{a, t} / q_{t}$ is replaced by $\bar{a} P_{a, t}^{(1)} / q_{t}$, where the (1) superscript indicates that this $P_{a, t}^{(1)}$ is the price of $\operatorname{good} a$ in the $L_{A}=1$ equilibrium. Similarly, the yellow dashed line in Panel (b) is $\mu_{\eta}$ from the $L_{A}=1$ equilibrium but with any prices of intermediate goods in (16) and (17) replaced by the prices from the competitive equilibrium.

These counterfactual drifts of $\eta$ demonstrate how the terms of trade hedge affects the rate of recovery to the stochastic steady state. In Panel (a), the change in the terms of trade caused by the AE's leverage constraint substantially increases $\mu_{\eta}$ when $\eta \in[0.1,0.2]$. However, the better terms of trade for the AE does not explain all of the change in the drift of $\eta$. The difference between the solid red line and dashed yellow line results from changes in equilibrium risk pricing caused by leverage constraints. Accounting for those changes approximately doubles $\mu_{\eta}$ for $\eta \in[0.1,0.2]$. Panel (b) reinforces this point. If one replaces the intermediate good price terms in $\mu_{\eta}$ from the $L_{A}=1$ equilibrium with prices from the competitive equilibrium, $\mu_{\eta}$ drops sharply but not to zero.

Panel (c) shows how these counterfactual drifts affect long-run stability. The solid blue and red lines are the stationary distributions from the competitive equilibrium and $L_{A}=1$ with the solid blue and red lines, respectively. The stationary distribution in the dashed yellow line results from using the counterfactual drift in Panel (a) and the volatility of $\eta$ in the competitive equilibrium. By just using the terms of trade from the $L_{A}=1$ equilibrium, the implied stationary distribution (dashed yellow) is almost the same as the $L_{A}=1$ distribution (solid red). In contrast, the stationary distribution in the dashed purple line results from using the counterfactual drift in Panel (b) and the volatility of $\eta$ in the $L_{A}=1$ equilibrium. While the drift generating the dashed purple distribution has the same volatility as the $L_{A}=1$ equilibrium, the distribution is only slightly more stable than the competitive equilibrium's, with a little less mass near $\eta=0$ and a little more mass for $\eta \in[0.1,0.2]$. The difference between these two distributions shows that the terms of trade hedge is the more important mechanism through which leverage constraints provide financial stability. Changing the terms of trade produces almost as much stability as the full $L_{A}=1$ equilibrium, 
but changing the volatility of equilibrium objects barely improves stability. The reason is that the terms of trade hedge make the stochastic steady state more stable. Given an adverse shock, tighter leverage constraints cause the terms of trade hedge to apply much closer to the optimal allocation of capital. This encourages a quicker return to "good times."

As further evidence, Panel (d) constructs two more counterfactual stationary distributions. To generate the dashed yellow distribution, we pair the drift of $\eta$ from the competitive equilibrium with the volatility of $\eta$ from the $L_{A}=1$ equilibrium, and to generate the dashed purple distribution, we pair the drift of $\eta$ from the $L_{A}=1$ equilibrium with the volatility of $\eta$ from the competitive equilibrium. The difference between the dashed yellow and purple lines demonstrates that the stabilizing force of leverage constraints operates through their effect on the terms of trade hedge. When we use the drift of $\eta$ from the competitive equilibrium, where the terms of trade hedge is relatively weak, the AE's long-run financial stability becomes worse. In contrast, when we only use the drift from the constrained equilibrium, the consequent stationary distribution is almost the same as the true constrained equilibrium.

\section{Numerical Method}

Here, we provide a description of the algorithm used to compute dynamics and welfare.

\section{C.1 Equations}

Rather than solve the ODEs implied by the drifts of $v_{A}$ and $v_{E}$ directly, we will utilize the false transient method and convert the system of ODEs into a system of PDEs with one time derivative and one "spatial" derivative. A natural way to add false time derivatives is the time-dependent version of Ito's lemma, which yields the new drifts

$$
\begin{aligned}
& \mu_{v A}=\frac{\partial_{t} v_{A}}{v_{A}}+\frac{\partial_{\eta} v_{A}}{v_{A}} \mu_{\eta} \eta+\frac{1}{2} \frac{\partial_{\eta \eta} v_{A}}{v_{A}} \eta^{2}\left\|\Sigma_{\eta}\right\|^{2} \\
& \mu_{v E}=\frac{\partial_{t} v_{E}}{v_{E}}+\frac{\partial_{\eta} v_{E}}{v_{E}} \mu_{\eta} \eta+\frac{1}{2} \frac{\partial_{\eta \eta} v_{E}}{v_{E}} \eta^{2}\left\|\Sigma_{\eta}\right\|^{2} .
\end{aligned}
$$


The equilibrium PDEs then are given by

$$
\begin{aligned}
\frac{\bar{a}\left(P_{a}-P_{b}\right)}{q}+\sigma \sigma_{q A}-\sigma \sigma_{q E}= & \gamma_{A} \varsigma_{A a}+\left(\gamma_{A}-1\right) \varsigma_{\xi A}-\gamma_{E} \varsigma_{E b}-\left(\gamma_{E}-1\right) \varsigma_{\zeta E} \\
\frac{\partial_{t} v_{A}}{v_{A}}+\frac{\partial_{\eta} v_{A}}{v_{A}} \mu_{\eta} \eta+\frac{1}{2} \frac{\partial_{\eta \eta} v_{A}}{v_{A}} \eta^{2}\left\|\Sigma_{\eta}\right\|^{2}= & \frac{\gamma_{A}}{2}\left[\left(\psi_{A} \sigma_{A}+\sigma_{v_{A} A}\right)^{2}+\left(\psi_{E} \sigma_{E}+\sigma_{v_{A} E}\right)^{2}\right] \\
& +\rho\left(\log \left(v_{A}\right)-\log (\rho q \eta)\right)-(\Phi(\boldsymbol{l})-\delta) \\
& -\psi_{A} \sigma_{A} \sigma_{v_{A} A}-\psi_{E} \sigma_{E} \sigma_{v_{A} E} \\
\frac{\partial_{t} v_{E}}{v_{E}}+\frac{\partial_{\eta} v_{E}}{v_{E}} \mu_{\eta} \eta+\frac{1}{2} \frac{\partial_{\eta \eta} v_{E}}{v_{E}} \eta^{2}\left\|\Sigma_{\eta}\right\|^{2}= & \frac{\gamma_{E}}{2}\left[\left(\psi_{A} \sigma_{A}+\sigma_{v_{E} A}\right)^{2}+\left(\psi_{E} \sigma_{E}+\sigma_{v_{E} E}\right)^{2}\right] \\
& +\rho\left(\log \left(v_{E}\right)-\log (\rho q(1-\eta))\right)-(\Phi(\boldsymbol{l})-\delta) \\
& -\psi_{A} \sigma_{A} \sigma_{v_{E} A}-\psi_{E} \sigma_{E} \sigma_{v_{E} E} .
\end{aligned}
$$

We pin down capital allocations using the conditions

$$
\begin{aligned}
q & =\frac{\kappa\left(\bar{a} \psi_{A a}+\underline{a} \psi_{E a}\right)^{\alpha}\left(\underline{a} \psi_{A b}+\bar{a} \psi_{E b}\right)^{1-\alpha}+1}{\rho \kappa+\lambda} \\
1 & =\psi_{A a}+\psi_{A b}+\psi_{E a}+\psi_{E b} \\
\frac{a}{\bar{a}} & \leq \frac{P_{a}}{P_{b}} \leq \frac{\bar{a}}{\underline{a}} \\
\psi_{A} & \leq\left(1+L_{A}\right) \eta, \quad \psi_{E} \leq\left(1+L_{E}\right)(1-\eta),
\end{aligned}
$$

where $L_{i}$ is presumed infinite if Country $i$ does not impose leverage constraints.

The volatilities for a generic endogenous object $f$ take the form

$$
\begin{aligned}
\sigma_{f A} & =\frac{\partial_{\eta} f}{f} \sigma_{\eta A} \eta \\
\sigma_{f E} & =\frac{\partial_{\eta} f}{f} \sigma_{\eta E} \eta
\end{aligned}
$$

The endogenous objects whose volatilities are required to compute equilibrium are $q$, $v_{A}$, and 
$v_{E}$. We also compute the volatilities for $\xi$ and $\zeta$ using the identities

$$
\begin{aligned}
\sigma_{\xi_{A}} & =\sigma_{v_{A} A}-\sigma_{\eta A}-\sigma_{q A} \\
\sigma_{\xi_{E}} & =\sigma_{v_{A} E}-\sigma_{\eta E}-\sigma_{q E} \\
\sigma_{\zeta A} & =\sigma_{v_{E} A}+\frac{\eta}{1-\eta} \sigma_{\eta A}-\sigma_{q A} \\
\sigma_{\zeta E} & =\sigma_{v_{E} E}+\frac{\eta}{1-\eta} \sigma_{\eta E}-\sigma_{q E}
\end{aligned}
$$

\section{C.2 Algorithms}

\section{C.2.1 Solving Equilibrium}

We solve for $q, v_{A}$, and $v_{E}$ using the method of false transients. We adopt the solution procedure outlined in Brunnermeier and Sannikov (2016b) and split the solution method into a static step and a time step. There are a variety of ways to reduce the dimensionality of the state space to a finite one. We choose finite differences, although other methods like Chebyshev collocation would be viable as well. In our description of the algorithm, assume all derivatives of $q, v_{A}$, and $v_{E}$ with respect to $\eta$ have been approximated using finite differences ${ }^{24}$ To start the algorithm, the user needs to specify initial guesses for $v_{A}$ and $v_{E}$. We initially use similar guesses to those proposed by Brunnermeier and Sannikov (2016b) to solve at least one equilibrium. Once we have solved some equilibria, we save those value functions and use them as initial guesses for other equilibria in our numerical exercises. The static step goes first, followed by the time step. Repeat until convergence is achieved for $v_{A}$ and $v_{E} \cdot 25$

Static Step. Assume we know $v_{A}$ and $v_{E}$.

1. Use the stationary economies when $\eta=0$ and $\eta=1$ to provide boundary conditions for $q$ at $\eta=0$ and $\eta=1$.

2. Convert the conditions pinning down capital allocations and the first-order differential equa-

\footnotetext{
${ }^{24} \mathrm{We}$ apply an upwind difference scheme to $v_{A}$ and $v_{E}$ since the state space has only one non-time variable.

${ }^{25}$ We choose a tolerance of $10^{-6}$ for the maximum pointwise change in $v_{A}$ and $v_{E}$.
} 
tion (by approximating $q^{\prime}$ with finite differences) into functions of $q$.

3. Apply a nonlinear equation solver to compute $q$.

4. Back out capital allocations, goods prices, $\mu_{\eta}, \Sigma_{\eta}$, volatilities of equilibrium objects, the growth rate of capital, and other quantities of interest (e.g. output levels).

Time Step. Assume we are given $q$, capital allocations, the capital growth rate, $\mu_{\eta}, \Sigma_{\eta}$, and the volatilities of $v_{A}$ and $v_{E}$. Then take a small time step back from $t$ to $t-\Delta t$ and solve the PDE backwards in time 26

\section{C.2.2 Solving for the Coordinated and Uncoordinated Equilibrium}

We arrive at most of our results by calculating ex-ante welfare over a fine grid of many pairs of leverage constraints and interpolating to determine optimal and Nash policies. One of the nodes in this grid is always $\left(L_{A}, L_{E}\right)=(0,0)$. In addition, we use an iterative procedure described below to verify that Nash is $(0,0)$. The algorithm is essentially a fixed point iteration, and convergence occurs when both countries are playing best responses.

1. Initialize $L_{A} \stackrel{27}{27}$

2. Do until convergence to a Nash equilibrium

(i) Fixing $L_{A}$, compute ex-ante welfare using a grid of leverage constraints for the EME. Interpolate and select the optimal $L_{E}$.

(ii) Fixing $L_{E}$, compute ex-ante welfare using a grid of leverage constraints for the AE. Interpolate and select the optimal $L_{A}$.

\footnotetext{
${ }^{26}$ Since the derivatives w.r.t. $\eta$ have been approximated with finite differences, solving the PDE reduces to solving an ordinary differential equation. The size of the time step may also be iteratively updated.

${ }^{27}$ To check our results do not depend on the initial $L_{A}$, we initialize many $L_{A}$.
} 


\section{International Negotiations and Bargaining Power}

We can apply the insights of our theoretical model to the case of international financial regulations. Global recommendations for financial regulation reflect agreements reached as the outcome of (possibly complicated) negotiations among countries. Because countries can always revert to Nash policies unilaterally, our results suggest that AEs (especially the U.S.) have strong bargaining power when negotiating international agreements. Crucially, the incentives for coordination differ between AEs and EMEs. AEs prefer Nash policies because they mitigate exposure to long-run financial instability. In contrast, EMEs want AEs to provide intermediation, which destabilizes AEs. To obtain intermediation, EMEs need to compensate AEs, so during negotiations, greater attention will be paid toward the concerns of AEs. In other words, the Pareto weight assigned to the welfare of AEs will be relatively large. As a proof of concept, Appendix D.1 develops a simple example where the AE in our model adopts open an capital account and allows the EME to maintain closed a capital account if the EME pays the AE a wealth transfer. The transfer Pareto dominates the Nash equilibrium, but, relative to the competitive equilibrium, only the $\mathrm{AE}$ is better off.

While we do not claim that our model exactly explains the policy decisions of AEs and EMEs, our model does predict recent empirical facts. Barth et al. (2013) show that after the financial crisis the U.S. has tightened regulations (e.g. on bank capital) more relative to many of its peers, reflecting the unilateral incentives which lead to the excessively stringent policies in our model's Nash equilibrium. In the 1990s, AEs held the "Washington Consensus" view and pressured EMEs to adopt free capital accounts. Presumably, AEs perceived economic benefits from these actions. This outcome reflects our model's prediction that a country's welfare increases with the laxness of the other country's prudential controls because it increases the probability of being relatively rich. Indeed, market liberalization in the 1990s precipitated financial crises in several EMEs and likely caused wealth redistribution toward AEs.

To be fair, prior to the financial crisis, AEs increased their international financial integration more quickly than EMEs. Over the period 1990-2004, for example, Lane and Milesi-Ferretti (2007) show that the sum of foreign assets and liabilities to GDP rose faster in AEs. This evidence appears to contradict our model in the sense that in older historical periods, AEs have developed 
freer capital accounts, not tighter ones. However, this argument assumes that world outcomes must be an uncoordinated Nash equilibrium. We have also shown that financial asymmetries generate asymmetric objectives. EMEs want AEs to provide intermediation, but AEs want to avoid bearing systemic risk. This asymmetry gives AEs, on the margin, more bargaining power in international policy coordination. It is reasonable that AEs may open their capital accounts to EMEs in exchange for economic benefits from EMEs.

The experience of EMEs in the design of international financial regulations also corroborates our prediction. The Basel standards are a key component of international financial regulations, but only after the financial crisis were EMEs included in discussions to construct these standards. Walter (2015) and Shimizu (2013) observe that this narrow membership led to a focus during Basel III on regulations most relevant to banks residing in developed countries. Similar concerns have been expressed about the previous Basel accords as well.

In the following two sections, we present a simple example showing how financial asymmetry could lead to policies that primarily benefit the AE and then discuss how our model's predictions are consistent with commentary about the ways in which AEs both control and benefit the most from recent international financial regulations.

\section{D.1 Bargaining Power Example}

This section expands the space of permissible actions by the EME to model this bargaining power in a simple reduced form. The EME can impose a wealth tax on its agents at a rate $\tau$ and use those taxes to pay the AE when $\eta$ is below some threshold $\eta_{\tau}$. At time $t=0$, the EME and AE can also implement a one-time wealth transfer to achieve an initial wealth allocation. It is in the EME's interest to pay this tax and agree to the initial wealth redistribution because a sufficiently generous tax and redistribution can make the $\mathrm{AE}$ willing to raise its leverage constraint above zero. One could interpret these policies as insurance paid to the AE by the EME in exchange for the AE's providing intermediation. The tax modifies the laws of motion for $\eta(16$ and (17) by adding the term $\tau(1-\eta) / \eta$ to $\mu_{\eta}$ when $\eta<\eta_{\tau}$. We do not interpret the wealth tax literally but instead utilize it as a modeling device that represents the capacity of AEs to negotiate international policies to 
their benefit.

Since we aim mainly to provide an example, we do not conduct an exhaustive search for the optimal policy the AE and EME can obtain through redistributive policies. We choose the parameters $\eta_{\tau}=0.5, \tau=40 \mathrm{bp}, \eta_{0}=0.4934, L_{A}$ never binding, and $L_{E}=0$. With these choices, the AE obtains a consumption equivalent welfare gain of 55bp while the EME acquires a 5.8bp increase. When this option is available, both countries strictly prefer to cooperate on this equilibrium instead of the Nash equilibrium. A simple way to add an explicit bargaining process is assume countries play a finite-horizon sequential-offer bargaining game before "time begins." Their payoffs are the value functions at $t=0$ in the infinite-horizon economy under the policies prescribed by the vector $\left(\eta_{\tau}, \tau, \eta_{0}, L_{A}, L_{E}\right)$. For example, countries may sequentially propose an $\eta_{0}$ fixing $\eta_{\tau}=0.5$, $\tau=40 \mathrm{bp}, L_{A}$ never binding, and $L_{E}=0$. For each round that countries fail to agree, the final pay offs decrease. One way to literally implement the decrease in final pay offs is to assume a fraction of the initial aggregate capital stock is destroyed for each round of failed coordination. However, whereas these policies improve the AE's welfare outcomes even more, they do not help the EME very much. Relative to the competitive equilibrium, the EME still suffers a welfare decrease of $19.1 \%$ in consumption equivalents.

\section{D.2 Negotiations of International Agreements}

Advanced economies (AEs) hold substantial influence over the governance and direction of many international economic organizations, and only recently have emerging market economies (EMEs) begun to take significant leadership in the writing of international regulatory standards. As of September 2018, the U.S., Japan, Germany, France, and the U.K. alone hold 36\% of the allotted votes on the IMF Executive Board. Moreover, every president of the World Bank was American, every managing director of the IMF has been an European, and every chairman of the Bank for International Settlements (BIS) has been from an AE. Out of 20 members on BIS's board of directors, 16 come from AEs. Furthermore, it was not until after the Financial Crisis that EMEs were included in regulatory discussions by the Basel Committee on Banking Supervision (BCBS). Similarly, the G20 replaced the G8 as the main body for international economic cooperation only 
in 2009.

Despite greater formal representation for EMEs, AEs continue to dominate the activities of these organizations when it comes to financial regulation. Walter (2015) finds evidence that EMEs still do not participate very much in the creation of financial rules like Basel III, which may be explained by mismatch between the agendas of these regulatory bodies and EME interests as well as EME's comparative lack of resources and experiences navigating international financial regulation. Bair (2012) relates an account of the Basel III discussions about leverage ratios, portraying the debate as two-died between an opposing group (Germany, France, and Japan), and a supporting group (the U.S., U.K., Canada, Switzerland, the Netherlands, Sweden, and most Basel Committee members). Notably, the account made no mention of EMEs. These claims are corroborated by Watanagase (2013), who alleges that "most proposed regulations [by Basel III] are formulated and calibrated mainly based on advanced markets' contexts and data, which can be significantly different from [EMEs]."

Existing explanations for this outcome can be broadly characterized as follows: AEs hold significant bargaining power during the negotiation of new rules because they have greater market size and more expertise with financial systems and regulation. ${ }^{28}$ As a result, AEs are able to shape the international financial system to better fit their preferences. Our paper has proposed an additional channel through which AEs have greater bargaining power over EMEs: the key role AEs play in bearing financial instability. AEs with developed financial systems can better intermediate risky investments through the issuance of debt liabilities, but doing so exposes AEs to a greater risk of financial crisis.

Our results corroborate and supplement commentary about the ways in which AEs both control and benefit the most from recent international financial regulations. Indeed, there is some concern that AEs design global macroprudential and capital account policies that are not well-suited, at a micro level, for EMEs. 29 When creating international regulatory standards in response to the

\footnotetext{
${ }^{28}$ For example, in a speech on behalf of the BIS, White (2001) justified the sparse membership of the BCBS during Basel II on the grounds that representatives from AEs had greater expertise due to their more developed financial systems. Walter (2015) discusses evidence for why EMEs may have limited knowledge on constructing and implementing international financial regulations. See chapter 2 of Drezner (2007) for a game-theoretic model demonstrating how AEs can take advantage of their economic power and market size to influence international rule-setting.

${ }^{29}$ In the popular press, Taylor 2010) writing in the Financial Times emphasizes that Basel III regulations are too
} 
financial crises of the 1990s, developed countries either vested new powers in organizations with predominantly AE membership or constructed new institutions like the Financial Stability Forum rather than use established organizations like the IMF. The latter choice reflected the fact that on the IMF Executive Board, the precedent is to make a decision only when consensus has been achieved. If AEs had chosen to vest the IMF with these powers, then they would have had to consider the concerns of EMEs when writing new international financial regulations. Through the creation of new institutions and the use of existing ones with membership dominated by their own representives-whether this was the desired intention or not-AEs could write regulations based primarily on their own preferences. For more details, see chapter 5 in Drezner (2007).

Despite these circumstances, the standards written by these bodies still became de facto international standards for EMEs. AEs linked these standards to participation in international institutions and to market mechanisms. Access to resources from organizations like the IMF began to require compliance with financial regulatory standards written by AE-dominated organizations like the Financial Stability Forum. Even though developing countries did not participate in the creation of those standards, they nevertheless had to follow them to acquire needed resources. Beck et al. (2018) finds evidence that EMEs, when seeking advice from the IMF and World Bank, have been advised or encouraged to adopt parts of Basel II and III, despite these standards not being official requirements. Markets also reinforced the need to comply with these regulations. Failure to demonstrate compliance would generate negative signals in financial markets. To facilitate expansion by both EME banks into foreign markets and AE banks into EME markets, many EMEs adopted Basel II and Basell III because they are de facto international regulatory standards, as Beck et al. (2018) notes.

Because EMEs were included in Basel discussions only after the Financial Crisis, Basel III still tended to focus on regulations most relevnt to banks residing in AEs. Shimizu (2013) notes that some of the regulations, like the more stringent capital adequacy regulation, may impair economic growth in Asian economies without substantially improving financial stability because of the differences in the structure of their financial systems. Historically, the introduction of Basel regulatory standards sharply contracted the supply and weakened the demand for credit in Japan, complex for emerging markets to implement well (e.g. risk management offices are just not sophisticated enough). 
contributing to the country's "lost decade." Substantial portions of Shimizu (2013) also criticize Basel's capital adequacy regulation as ineffective for addressing systemic risk and as a major cause of Japan's credit crunch during the 1990s.

The implementation of Basel III's leverage ratio may similarly penalize EMEs. Most banks in Asian EMEs focus on supplying traditional financial products and especially trade finance. For example, Watanagase (2013) reports that at the time of the Financial Crisis, only 0.1 percent of total assets held by the Thai banking sector were invested in products related to collateralized debt obligations. Basel III's leverage ratio, however, could render traditional financial products like trade finance more costly than holding off-balance-sheet items like derivatives. On the margin, the comparatively higher cost of traditional products may incentivize financial institutions to invest more of their portfolio in sophisticated and riskier products.

Basel III and unilateral regulatory reforms in response to the financial crisis (e.g., ring-fencing in the UK and the Volcker rule in the US) may also reduce the volume of cross-border transactions with EME banks due to higher costs, adversely impacting EMEs. Alford (2013) worries that, because of additional supervision, systemically important financial institutions (SIFI) may reduce their investment in emerging markets. Beck (2018) discusses some of the channels through which fund flows may be reduced.

While Basel III focused on many rules-based regulations, EMEs tend to favor more discretionary regulations because their financial systems are different from both each others' and the financial systems of AEs. Shimizu (2013) pushes Basel III to allow flexibility in the national implementation of its standards because rules targeting the causes of financial crises in Western AEs like the U.S. may not be effective in Asian economies and could constrict the flow of funds needed for growth. Other central banks, notably the Reserve Bank of India, have also seen success using dynamic sector risk-weight adjustment, where regulators use their discretion to identify the systemic risk of different asset classes and re-weight their supervision accordingly (see Acharya (2013)). Watanagase (2013) advocates discretionary risk-based supervision as an essential component of financial regulations because exceedingly rules-based regulations may not adapt to new technologies and changing sources of systemic risk. Knowledge of the linkages between the finan- 
cial sector and the real economy is also limited, so regulatory rules may not be complete in their scope, making discretion a necessary dimension for policymakers.

\section{E Robustness}

Here, we check the robustness of our results. We show that zero leverage constraints still comprise a Nash equilibrium in the case of symmetric risk aversion coefficients. We also check that our main results are not sensitive to our choices of risk aversion coefficients nor the gains from specialization in trade. For all the parameters we consider, we still obtain a Nash equilibrium in which both countries impose zero leverage constraints, and the AE gains at the expense of the EME, relative to the competitive equilibrium. Below, we report consumption equivalent welfare changes from the competitive equilibrium to Nash for each country.

\section{E.1 Symmetric Economy}

We set $\gamma_{A}=\gamma_{E}=1.5$ so that our coefficients are equally between 1 and 2 , but this choice is not essential. We still obtain a Nash equilibrium of $\left(L_{A}, L_{E}\right)=(0,0)$. However, because there is no asymmetry in risk aversion coefficients, Nash does not harm one country at the expense of the other. Relative to the competitive equilibrium, Nash policies produce welfare gains of $7.01 \%$ in consumption equivalents for both countries. Optimal policies are slightly positive at $L_{A}=L_{E} \approx$ 90bp, but the gains relative to Nash are small (on the order of a couple basis points). In a symmetric world, the strategic incentives align very closely with the socially optimal ones because neither country demands the other's intermediation relatively more. It is precisely an asymmetry in risk tolerance that lies at the crux of our analysis.

\section{E.2 Risk Aversion}

Table 3 reports the consumption equivalent change from the competitive equilibrium to Nash equilibrium for each country while varying our choices for risk aversion coefficients. The more asymmetric risk aversion is, the larger the increase in welfare that the AE obtains in Nash and the larger 
Risk Aversion Welfare Change for AE Welfare Change for E Debt/Output Ratio

\begin{tabular}{cccc}
\hline & & & \\
$\gamma_{A}=1.4, \gamma_{E}=1.6$ & $11.0 \%$ & $3.31 \%$ & -.271 \\
$\gamma_{A}=1.25, \gamma_{E}=1.75$ & $16.9 \%$ & $-1.38 \%$ & -1.82 \\
$\gamma_{E}=1.9$ & $24.8 \%$ & $-6.62 \%$ & -3.37 \\
Original & $26.8 \%$ & $-7.62 \%$ & -3.38 \\
$\gamma_{A}=1.01$ & $28.2 \%$ & $-8.34 \%$ & -3.89 \\
$\gamma_{E}=2.1$ & $28.6 \%$ & $-8.53 \%$ & -3.89 \\
$\gamma_{E}=3$ & $43.2 \%$ & $-14.6 \%$ & -5.43 \\
\hline \hline
\end{tabular}

Table 3: This table displays the consumption equivalent welfare change from the competitive equilibrium to Nash while varying the degree of asymmetry in risk aversion. We report the original calibration (for comparison) and the risk aversion coefficient(s) we change from our original calibration. For example, the third row has only $\gamma_{E}=1.9$, so we maintain $\gamma_{A}=1.05$ for that row's calculations. The different cases are reported in order of the absolute difference in risk aversion.

the decrease in welfare that the EME experiences relative to the competitive equilibrium. This result reinforces our point that the EME wants the $\mathrm{AE}$ to bear sources of long-run financial instability because the $\mathrm{AE}$ has relatively higher risk tolerance. A larger asymmetry in risk aversion leads to greater demand by the EME for intermediation by the AE. When the AE denies that intermediation, it produces larger gains for itself while worsening the welfare loss suffered by the EME.

However, if the asymmetry in risk aversion is sufficiently small, then Nash equilibrium improves outcomes for both countries. This occurs in the case of $\left(\gamma_{A}, \gamma_{E}\right)=(1.4,1.6)$. The intuition is that when the EME's risk tolerance is close enough to the AE's, then the EME's demand for intermediation by the $\mathrm{AE}$ is relatively low, and the benefits from greater long-run financial stability are relatively high. Although both countries obtain better outcomes, the welfare gains are still asymmetric. the AE's welfare gain is greater than the gain in the symmetric economy while the EME's gain is lower. This difference emerges due to the mechanism highlighted by our original calibration. With an asymmetry in risk aversion, the country with lower risk aversion ends up bearing the burden of long-run instability relatively more, so zero leverage constraints are more 
$\underline{a} \quad$ Welfare Change for $\mathrm{AE}$ Welfare Change for $\mathrm{E}$

\begin{tabular}{lll}
\hline \hline & & \\
0.75 & $26.7 \%$ & $-7.62 \%$ \\
0.85 & $26.8 \%$ & $-7.62 \%$ \\
0.95 & $27.9 \%$ & $-7.63 \%$ \\
& & \\
\hline \hline
\end{tabular}

Table 4: This table displays the consumption equivalent welfare change from the competitive equilibrium to Nash while varying the degree of productivity gains from specialization.

beneficial for that country.

The final column of Table 3 reports the debt to domestic output ratio in the stochastic steady state. This column gives some sense about the degree of imbalances in the stochastic steady state as the asymmetry in risk aversion widens. The wider the asymmetry, the more leveraged the $\mathrm{AE}$ becomes in the steady state. This occurs for two reasons. First, the AE becomes more leveraged in general since it has a higher risk tolerance. Second, the stochastic steady state shifts toward zero as the $\mathrm{AE}$ because the $\mathrm{AE}$ is able to lever up and achieve nearly optimal capital allocations much quicker.

\section{E.3 Gains from Specialization}

Table 4 reports the consumption equivalent change from the competitive equilibrium to Nash equilibrium for each country. Generally, the smaller the gains from specialization (i.e. the greater $\underline{a}$ is), the larger the increase in welfare that the AE obtains in Nash and the larger the decrease in welfare that the EME experiences relative to the competitive equilibrium. The size of the changes, however, are not that different across the different parameter choices. 


\section{F Extension: Location and Ownership Shocks}

Here, we show that our baseline model generates similar risk premia to a model that includes shocks dependent on the country location and country of ownership. In Section F.5, we show that with log flow utility, there exists a parametrization of the extended model such that the risk premia required by $\mathrm{AE}$ agents are the same as in the original model.

\section{F.1 Setup}

For brevity, we do not mention details that do not change relative to the baseline model, such as preferences.

Technology. Without loss of generality, we assume the aggregate stock of capital at time $t=0$ is equally distributed between the $\mathrm{AE}$ and the EME. Capital produced from internal investment in a given country remains located in that country. Since the rate of internal investment is determined by Tobin's q, every agent chooses the same rate, so capital grows at the same rate in the aggregate in either country. As a result, capital remains equally distributed between the AE and the EME for all time.

Define $\psi_{i j k, t} \psi_{i j k, t}, \psi_{i j k, t}$, and $\psi_{i j k, t}$ to be the share of capital owned by Country $i=A, E$ used to produce good $j=a, b$ with capital located in Country $k=A, E$. market clearing for capital implies

$$
\sum_{i=A, E} \sum_{j=a, b} \sum_{k=A, E} \psi_{i j k, t}=1
$$

We additionally define

$$
\begin{aligned}
\psi_{i, t} & =\sum_{j=a, b} \sum_{k=A, E} \psi_{i j k, t} \\
\psi_{i k, t} & =\sum_{j=a, b} \psi_{i j k, t}
\end{aligned}
$$

For a single agent located in Country $i$ using capital in Country $k$, the capital that they own evolves 
according to

$$
\frac{d k_{t}}{k_{t}}=\left(\Phi\left(l_{t}\right)-\delta\right) d t+\sigma_{i O} d W_{i O, t}+\sigma_{k L} d W_{k L, t}
$$

In the interest of generality, we also let $\sigma_{i O}$ depend on the user's country of origin. In total, there are now four Brownian motions: $W_{A O, t}, W_{E O, t}, W_{A L, t}$ and $W_{E L, t}$. We assume these Brownian motions are mutually independent. The first two shocks are shocks to capital owned by the AE and the EME, respectively. The latter two shocks are shocks to capital located in the AE and the EME, respectively. Aggregating together, the stock of world capital $K_{t}$ evolves according to

$$
\begin{aligned}
\frac{d K_{t}}{K_{t}}= & \left.\left(\Phi\left(\imath_{t}\right)-\delta\right) d t\right)+\psi_{A, t} \sigma_{A O} d W_{A O, t}+\psi_{E, t} \sigma_{E} d W_{E O, t} \\
& +\left(\psi_{A A, t}+\psi_{E A, t}\right) \sigma_{A L} d W_{A L, t}+\left(\psi_{A E, t}+\psi_{E E, t}\right) \sigma_{E L} d W_{A E, t}
\end{aligned}
$$

We conjecture that the price of capital follows

$$
\frac{d q_{t}}{q_{t}}=\mu_{q, t} d t+\sigma_{q A O, t} d W_{A O, t}+\sigma_{q B O, t} d W_{B O, t}+\sigma_{q A L, t} d W_{A L, t}+\sigma_{q E L, t} d W_{E L, t} .
$$

Returns from Capital. The returns for the AE investing in good $a$ using capital located in the $\mathrm{AE}$ and capital located in the EME are given by, respectively,

$$
\begin{aligned}
d r_{t}^{A a A}= & \left(\frac{\bar{a} P_{a, t}}{q_{t}}+\mu_{q, t}+\Phi\left(l_{t}\right)-\delta+\sigma_{A O} \sigma_{q A O, t}+\sigma_{A L} \sigma_{q A L, t}\right) d t \\
& +\left(\sigma_{A O}+\sigma_{q A O, t}\right) d W_{A O, t}+\sigma_{q E O, t} d W_{E O, t}+\left(\sigma_{A L}+\sigma_{q A L, t}\right) d W_{A L, t} \\
d r_{t}^{A a E}= & \left(\frac{\bar{a} P_{a, t}}{q_{t}}+\mu_{q, t}+\Phi\left(l_{t}\right)-\delta+\sigma_{A O} \sigma_{q A O, t}+\sigma_{E L} \sigma_{q E L, t}\right) d t \\
& +\left(\sigma_{A O}+\sigma_{q A O, t}\right) d W_{A O, t}+\sigma_{q E O, t} d W_{E O, t}+\left(\sigma_{A E}+\sigma_{q A E, t}\right) d W_{A E, t}
\end{aligned}
$$

The AE can also invest in good $a$ using capital located in the EME as well as in good $b$ using capital located in the AE or the EME. Similar returns equations hold for agents in the EME. It will be convenient to let $\mathbf{W}_{t}=\left[W_{A O, t}, W_{E O, t} W_{A L, t} W_{E L, t}\right]^{T}$ stack all exogenous shocks and let $\Sigma_{i j k, t}$ denote the volatility vector of $d r_{t}^{i j k}$ so that $\Sigma_{i j k, t} \cdot d \mathbf{W}_{t}$ gives the volatility of $d r_{t}^{i j k}$. 
Portfolio Choice. The portfolio choice problem is symmetric for agents in either country, so we only characterize the problem of $\mathrm{AE}$ agents. An agent in the $\mathrm{AE}$ chooses a consumption rate $c_{A, t}$ and portfolio weights $\left(x_{A a A, t}, x_{A a E, t}, x_{A b A, t}, x_{A b E, t}, 1-\sum_{j=a, b} \sum_{k=A, E} x_{A j k, t}\right)$. It will be convenient to let $x_{A, t}=\sum_{j=a, b} \sum_{k=A, E} x_{A j k, t}$ and $x_{A k, t}=\sum_{j=a, b} x_{A j k, t}$ for $k=A, E$. We may then write the evolution of net worth for $\mathrm{AE}$ agents as

$$
\frac{d n_{A, t}}{n_{A, t}}=\left(1-x_{A, t}\right) d r_{t}^{F}-\frac{c_{A, t}}{n_{A, t}} d t+\sum_{j=a, b} \sum_{k=A, E} x_{A j k, t} d r_{t}^{A j k}
$$

Denote by $\Sigma_{A j k, t}$ the volatility for $\mathrm{AE}$ agents to invest in the production of good $j=a, b$ with capital located in Country $k=A, E$. The risk premium required by $\mathrm{AE}$ agents is the covariance of these returns with the stochastic discount afctor of agents in the AE. In equilibrium, we obtain the following conditions:

$$
\begin{aligned}
& \mathbb{E}\left[d r_{t}^{A a A}\right]-d r_{t}^{F}=\gamma_{A}\left(x_{A A, t} \Sigma_{A a A, t}+x_{A E, t} \Sigma_{A a E, t}\right) \cdot \Sigma_{A a A, t}+\left(\gamma_{A}-1\right) \Sigma_{\xi, t} \cdot \Sigma_{A a A, t} \\
& \mathbb{E}\left[d r_{t}^{A a E}\right]-d r_{t}^{F}=\gamma_{A}\left(x_{A A, t} \Sigma_{A a A, t}+x_{A E, t} \Sigma_{A a E, t}\right) \cdot \Sigma_{A a E, t}+\left(\gamma_{A}-1\right) \Sigma_{\xi, t} \cdot \Sigma_{A a E, t} \\
& \mathbb{E}\left[d r_{t}^{A b A}\right]-d r_{t}^{F} \leq \gamma_{A}\left(x_{A A, t} \Sigma_{A a A, t}+x_{A E, t} \Sigma_{A a E, t}\right) \cdot \Sigma_{A a A, t}+\left(\gamma_{A}-1\right) \Sigma_{\xi, t} \cdot \Sigma_{A a A, t} \\
& \mathbb{E}\left[d r_{t}^{A b E}\right]-d r_{t}^{F} \leq \gamma_{A}\left(x_{A A, t} \Sigma_{A a E, t}+x_{A E, t} \Sigma_{A a E, t}\right) \cdot \Sigma_{A a E, t}+\left(\gamma_{A}-1\right) \Sigma_{\xi, t} \cdot \Sigma_{A a E, t} .
\end{aligned}
$$

The inequalities are sometimes slack because there are no short sales. The first two equalities must hold since the $\mathrm{AE}$ has a superior production technology for good $a$, and there are diversification benefits to using capital in both countries.

Taking the difference between the first two equalities yields

$$
\begin{aligned}
\sigma_{A L} \sigma_{q A L, t}-\sigma_{E L} \sigma_{q E L, t}= & \gamma_{A}\left(x_{A A, t} \Sigma_{A a A, t}+x_{A E, t} \Sigma_{A a E, t}\right)\left(\Sigma_{A a A, t}-\Sigma_{A a E, t}\right) \\
& +\left(\gamma_{A}-1\right) \Sigma_{\xi, t} \cdot\left(\Sigma_{A a A, t}-\Sigma_{A a E, t}\right) .
\end{aligned}
$$

Focusing on $\Sigma_{A a A, t}-\Sigma_{A a E, t}$, simplification yields the vector

$$
\left[0,0, \sigma_{A L}+\sigma_{q A L, t},-\sigma_{E L}-\sigma_{q A E, t}\right]^{T}
$$


We therefore obtain

$$
\begin{aligned}
\sigma_{A L} \sigma_{q A L, t}-\sigma_{E L} \sigma_{q E L, t}= & \gamma_{A}\left(x_{A A, t}\left(\sigma_{A L}+\sigma_{q A L, t}\right)^{2}-x_{A E, t}\left(\sigma_{E L}+\sigma_{q E L, t}\right)^{2}\right) \\
& +\left(\gamma_{A}-1\right)\left(\sigma_{\xi A A, t}\left(\sigma_{A L}+\sigma_{q A L, t}\right)-\sigma_{\xi A E, t}\left(\sigma_{E L}+\sigma_{q E L, t}\right)\right) .
\end{aligned}
$$

This equation pins down the allocation of the AE's capital located in the AE and in the EME.

\section{F.2 Law of Motion for $\eta$}

The aggregate law of motion for net worth in the $\mathrm{AE}$ is

$$
\frac{d N_{A, t}}{N_{A, t}}=d r_{t}^{F}-\frac{C_{A, t}}{N_{A, t}}+\sum_{j=a, b} \sum_{k=A, E}\left(d r_{t}^{A j k}-d r_{t}^{F}\right) .
$$

Notice that the asset-pricing conditions and the volatilities of returns for producing in the AE are the same for both goods $a$ and $b$. In other words, when AE agents produce both goods $a$ and $b$, we must have

$$
d r_{t}^{A a A}=d r_{t}^{A b A}, \quad d r_{t}^{A a E}=d r_{t}^{A b E}
$$

We can therefore write $d N_{A, t}$ as

$$
\frac{d N_{A, t}}{N_{A, t}}=d r_{t}^{F}-\frac{C_{A, t}}{N_{A, t}}+x_{A A, t}\left(d r_{t}^{A a A}-d r_{t}^{F}\right)+x_{A E, t}\left(d r_{t}^{A a E}-d r_{t}^{F}\right)
$$

Multiplying the returns terms by one yields

$$
\begin{aligned}
\frac{d N_{A, t}}{N_{A, t}} & =d r_{t}^{F}-\frac{C_{A, t}}{N_{A, t}}+x_{A, t} \frac{x_{A A, t}}{x_{A, t}}\left(d r_{t}^{A a A}-d r_{t}^{F}\right)+x_{A, t} \frac{x_{A E, t}}{x_{A, t}}\left(d r_{t}^{A a E}-d r_{t}^{F}\right) \\
& =d r_{t}^{F}-\frac{C_{A, t}}{N_{A, t}}+x_{A, t}\left(\frac{x_{A A, t}}{x_{A, t}}\left(d r_{t}^{A a A}-d r_{t}^{F}\right)+\frac{x_{A E, t}}{x_{A, t}}\left(d r_{t}^{A a E}-d r_{t}^{F}\right)\right)
\end{aligned}
$$

With the law of motion for $N_{A, t}$ written in this form, we can now show the similarity in risk premia between the present model and the baseline model. The risk premium for holding capital 
will take the same form as in the baseline model, which had

$$
\mathbb{E}\left[d r_{t}^{A a}\right]-d r_{t}^{F}=\gamma_{A} \varsigma_{A a, t}+\left(\gamma_{A}-1\right) \varsigma_{\xi A, t}
$$

Define $d r_{t}^{A}$ to be the return earned by $\mathrm{AE}$ agents per unit of capital held. This quantity is given by

$$
d r_{t}^{A}=\frac{x_{A A, t}}{x_{A, t}} d r_{t}^{A a A}+\frac{x_{A E, t}}{x_{A, t}} d r_{t}^{A a E}
$$

The return per unit of capital is an average of the returns $d r_{t}^{A a A}$ and $d r_{t}^{A a E}$, weighted by the relative shares of capital located in the $\mathrm{AE}$ and the EME. Taking expectations of the excess returns and substituting in the asset pricing conditions for AE agents yield

$$
\begin{aligned}
\mathbb{E}\left[d r_{t}^{A}\right]-d r_{t}^{F}= & \frac{x_{A A, t}}{x_{A, t}}\left(\mathbb{E}\left[d r_{t}^{A A A}\right]-d r_{t}^{F}\right)+\frac{x_{A E, t}}{x_{A, t}}\left(\mathbb{E}\left[d r_{t}^{A a E}\right]-d r_{t}^{F}\right) \\
= & \frac{x_{A A, t}}{x_{A, t}} \gamma_{A}\left(x_{A A, t} \Sigma_{A a A, t}+x_{A E, t} \Sigma_{A a E, t}\right) \cdot \Sigma_{A a A, t}+\left(\gamma_{A}-1\right) \Sigma_{\xi, t} \cdot \Sigma_{A a A, t} \\
& +\frac{x_{A E, t}}{x_{A, t}} \gamma_{A}\left(x_{A A, t} \Sigma_{A a A, t}+x_{A E, t} \Sigma_{A a E, t}\right) \cdot \Sigma_{A a E, t}+\left(\gamma_{A}-1\right) \Sigma_{\xi, t} \cdot \Sigma_{A a E, t} \\
= & \frac{x_{A A, t}}{x_{A, t}} \gamma_{A} x_{A, t}\left(\left(\sigma_{A O}+\sigma_{q A O, t}\right)^{2}+\sigma_{q E O, t}^{2}\right) \\
& +\frac{x_{A A, t}}{x_{A, t}}\left(\gamma_{A}-1\right)\left(\sigma_{\xi A O, t}\left(\sigma_{A O}+\sigma_{q A O, t}\right)+\sigma_{\xi E O, t} \sigma_{q E O, t}\right) \\
& +\frac{x_{A A, t}}{x_{A, t}}\left(\gamma_{A} x_{A A, t}\left(\sigma_{A L}+\sigma_{q A L, t}\right)^{2}+\left(\gamma_{A}-1\right) \sigma_{\xi A L, t}\left(\sigma_{A L}+\sigma_{q A L, t}\right)\right) \\
& +\frac{x_{A E, t}}{x_{A, t}} \gamma_{A} x_{A, t}\left(\left(\sigma_{A O}+\sigma_{q A O, t}\right)^{2}+\sigma_{q E O, t}^{2}\right) \\
& +\frac{x_{A E, t}}{x_{A, t}}\left(\gamma_{A}-1\right)\left(\sigma_{\xi A O, t}\left(\sigma_{A O}+\sigma_{q A O, t}\right)+\sigma_{\xi E O, t} \sigma_{q E O, t}\right) \\
& +\frac{x_{A E, t}}{x_{A, t}}\left(\gamma_{A} x_{A E, t}\left(\sigma_{E L}+\sigma_{q E L, t}\right)^{2}+\left(\gamma_{A}-1\right) \sigma_{\xi E L, t}\left(\sigma_{E L}+\sigma_{q E L, t}\right)\right) .
\end{aligned}
$$


Combining like terms obtains

$$
\begin{aligned}
\mathbb{E}\left[d r_{t}^{A}\right]-d r_{t}^{F}= & \gamma_{A} x_{A, t}\left(\left(\sigma_{A O}+\sigma_{q A O, t}\right)^{2}+\sigma_{q E O, t}^{2}\right) \\
& +\left(\gamma_{A}-1\right)\left(\sigma_{\xi A O, t}\left(\sigma_{A O}+\sigma_{q A O, t}\right)+\sigma_{\xi E O, t} \sigma_{q E O, t}\right) \\
& +\frac{x_{A A, t}}{x_{A, t}}\left(\gamma_{A} x_{A A, t}\left(\sigma_{A L}+\sigma_{q A L, t}\right)^{2}+\left(\gamma_{A}-1\right) \sigma_{\xi A L, t}\left(\sigma_{A L}+\sigma_{q A L, t}\right)\right) \\
& +\frac{x_{A E, t}}{x_{A, t}}\left(\gamma_{A} x_{A E, t}\left(\sigma_{E L}+\sigma_{q E L, t}\right)^{2}+\left(\gamma_{A}-1\right) \sigma_{\xi E L, t}\left(\sigma_{E L}+\sigma_{q E L, t}\right)\right) .
\end{aligned}
$$

Re-arrange terms to acquire

$$
\begin{aligned}
\mathbb{E}\left[d r_{t}^{A}\right]-d r_{t}^{F}= & \gamma_{A} x_{A, t}\left[\left(\sigma_{A O}+\sigma_{q A O, t}\right)^{2}+\frac{x_{A A, t}^{2}}{x_{A, t}^{2}}\left(\sigma_{A L}+\sigma_{q A L, t}\right)^{2}+\frac{x_{A E, t}^{2}}{x_{A, t}^{2}}\left(\sigma_{E L}+\sigma_{q E L, t}\right)^{2}+\sigma_{q E O, t}^{2}\right] \\
& +\left(\gamma_{A}-1\right) \sigma_{\xi A O, t}\left(\sigma_{A O}+\sigma_{q A O, t}\right) \\
& +\left(\gamma_{A}-1\right)\left[\frac{x_{A A, t}}{x_{A, t}} \sigma_{\xi A L, t}\left(\sigma_{A L}+\sigma_{q A L, t}\right)+\frac{x_{A E, t}}{x_{A, t}} \sigma_{\xi E L, t}\left(\sigma_{E L}+\sigma_{q E L, t}\right)+\sigma_{\xi E O, t} \sigma_{q E O, t}\right] .
\end{aligned}
$$

If we define

$$
\begin{aligned}
\varsigma_{A a, t}= & x_{A, t}\left[\left(\sigma_{A O}+\sigma_{q A O, t}\right)^{2}+\frac{x_{A A, t}^{2}}{x_{A, t}^{2}}\left(\sigma_{A L}+\sigma_{q A L, t}\right)^{2}+\frac{x_{A E, t}^{2}}{x_{A, t}^{2}}\left(\sigma_{E L}+\sigma_{q E L, t}\right)^{2}+\sigma_{q E O, t}^{2}\right] \\
\varsigma_{\xi A, t}= & \sigma_{\xi A O, t}\left(\sigma_{A O}+\sigma_{q A O, t}\right)+\frac{x_{A A, t}}{x_{A, t}} \sigma_{\xi A L, t}\left(\sigma_{A L}+\sigma_{q A L, t}\right) \\
& +\frac{x_{A E, t}}{x_{A, t}} \sigma_{\xi E L, t}\left(\sigma_{E L}+\sigma_{q E L, t}\right)+\sigma_{\xi E O, t} \sigma_{q E O, t},
\end{aligned}
$$

then the excess returns per unit of capital satisfies

$$
\mathbb{E}\left[d r_{t}^{A}\right]-d r_{t}^{F}=\gamma_{A} \varsigma_{A a, t}+\left(\gamma_{A}-1\right) \varsigma_{\xi A, t},
$$

as in the original model. The primary difference from the baseline model is that the terms $\varsigma_{A a, t}$ and $\varsigma_{\xi_{A, t}}$ are affected by the physical location of capital owned by AE agents and portfolio rebalancing conducted in response to location-specific shocks. 
Plugging (43) into the law of motion for $N_{A, t}$ yields

$$
\begin{aligned}
\frac{d N_{A, t}}{N_{A, t}}= & d r_{t}^{F}+\left(x_{A, t}\left(\gamma_{A} \varsigma_{A a, t}+\left(\gamma_{A}-1\right) \varsigma_{\xi A, t}\right)-\frac{C_{A, t}}{N_{A, t}}\right) d t \\
& +x_{A, t}\left(\sigma_{A O}+\sigma_{q A O, t}\right) d W_{A O, t}+x_{A, t} \sigma_{q E O, t} d W_{E O, t} \\
& +x_{A A, t}\left(\sigma_{A L}+\sigma_{q A L, t}\right) d W_{A L, t}+x_{A E, t}\left(\sigma_{E L}+\sigma_{q E L, t}\right) d W_{E L, t} .
\end{aligned}
$$

Let $\Sigma_{N A, t}$ denote the volatility vector for this process. By Ito's product rule,

$$
\frac{d\left(q_{t} K_{t}\right)}{q_{t} K_{t}}=\left(\mu_{q, t}+\Phi\left(l_{t}\right)-\delta+\Sigma_{q, t} \cdot \Sigma_{K, t}\right) d t+\left(\Sigma_{q, t}+\Sigma_{K, t}\right) \cdot d \mathbf{W}_{t} .
$$

Using the AE's asset-pricing conditions, we have

$$
\begin{aligned}
-\mu_{q, t}-\Phi\left(l_{t}\right)+\delta= & \frac{\bar{a} P_{a, t}}{q_{t}}+\sigma_{A O} \sigma_{q A O, t}+\sigma_{A L} \sigma_{q A L, t}-d r_{t}^{F} \\
& -\gamma_{A}\left(x_{A A, t} \Sigma_{A a A, t}+x_{A E, t} \Sigma_{A a E, t}\right) \cdot \Sigma_{A a A, t}-\left(\gamma_{A}-1\right) \Sigma_{\xi, t} \cdot \Sigma_{A a A, t} \\
-\mu_{q, t}-\Phi\left(l_{t}\right)+\delta= & \frac{\bar{a} P_{a, t}}{q_{t}}+\sigma_{A O} \sigma_{q A O, t}+\sigma_{E L} \sigma_{q E L, t}-d r_{t}^{F} \\
& -\gamma_{A}\left(x_{A A, t} \Sigma_{A a A, t}+x_{A E, t} \Sigma_{A a E, t}\right) \cdot \Sigma_{A a E, t}-\left(\gamma_{A}-1\right) \Sigma_{\xi, t} \cdot \Sigma_{A a E, t} .
\end{aligned}
$$

By Ito's lemma, we have

$$
\frac{d\left(1 /\left(q_{t} K_{t}\right)\right)}{1 /\left(q_{t} K_{t}\right)}=\left(\left\|\Sigma_{q, t}+\Sigma_{K, t}\right\|^{2}-\mu_{q, t}-\Phi\left(l_{t}\right)+\delta-\Sigma_{q, t} \cdot \Sigma_{K, t}\right) d t-\left(\Sigma_{q, t}+\Sigma_{K, t}\right) \cdot d \mathbf{W}_{t} .
$$

By Ito's product rule,

$$
\begin{aligned}
\frac{d \eta_{t}}{\eta_{t}}= & \left(d r_{t}^{F}-\mu_{q, t}-\Phi\left(\boldsymbol{l}_{t}\right)+\delta+\left\|\Sigma_{q, t}+\Sigma_{K, t}\right\|^{2}-\Sigma_{q, t} \cdot \Sigma_{K, t}-\frac{C_{A, t}}{N_{A, t}}\right) d t \\
& +\left(x_{A, t}\left(\gamma_{A} \varsigma_{A a, t}+\left(\gamma_{A}-1\right) \varsigma_{\xi A, t}\right)-\Sigma_{N A, t} \cdot\left(\Sigma_{q, t}+\Sigma_{K, t}\right)\right) d t \\
& +\left(\Sigma_{N A, t}-\Sigma_{q, t}-\Sigma_{K, t}\right) \cdot d \mathbf{W}_{t} .
\end{aligned}
$$


Notice that $x_{A A, t}+x_{A E, t}=x_{A, t}$ implies $x_{A A, t} / x_{A, t}+x_{A E, t} / x_{A, t}=1$. Thus, we have

$$
\begin{aligned}
& -\mu_{q, t}-\Phi\left(\boldsymbol{l}_{t}\right)+\delta \\
= & \frac{x_{A A, t}}{x_{A, t}}\left(-\mu_{q, t}-\Phi\left(\boldsymbol{l}_{t}\right)+\delta\right)+\frac{x_{A E, t}}{x_{A, t}}\left(-\mu_{q, t}-\Phi\left(\boldsymbol{l}_{t}\right)+\delta\right) \\
= & \frac{\bar{a} P_{a, t}}{q_{t}}-d r_{t}^{F}+\sigma_{A O} \sigma_{q A O, t} \\
& -\frac{x_{A A, t}}{x_{A, t}}\left[\gamma_{A}\left(x_{A A, t} \Sigma_{A a A, t}+x_{A E, t} \Sigma_{A a E, t}\right) \cdot \Sigma_{A a A, t}+\left(\gamma_{A}-1\right) \Sigma_{\xi, t} \cdot \Sigma_{A a A, t}-\sigma_{A L} \sigma_{q A L, t}\right] \\
& -\frac{x_{A E, t}}{x_{A, t}}\left[\gamma_{A}\left(x_{A A, t} \Sigma_{A a A, t}+x_{A E, t} \Sigma_{A a E, t}\right) \cdot \Sigma_{A a E, t}+\left(\gamma_{A}-1\right) \Sigma_{\xi, t} \cdot \Sigma_{A a E, t}-\sigma_{E L} \sigma_{q E L, t}\right] \\
= & \frac{\bar{a} P_{a, t}}{q_{t}}-d r_{t}^{F}+\sigma_{A O} \sigma_{q A O, t}+\frac{x_{A A, t}}{x_{A, t}} \sigma_{A L} \sigma_{q A L, t}+\frac{x_{A E, t}}{x_{A, t}} \sigma_{E L} \sigma_{q E L, t}-\gamma_{A} \varsigma_{A a, t}-\left(\gamma_{A}-1\right) \varsigma_{\xi A, t} .
\end{aligned}
$$

Additionally,

$$
\begin{aligned}
\Sigma_{q, t} \cdot \Sigma_{K, t}= & \psi_{A, t} \sigma_{A O} \sigma_{q A O, t}+\psi_{E, t} \sigma_{E O} \sigma_{q E O, t} \\
& +\left(\psi_{A A, t}+\psi_{E A, t}\right) \sigma_{A L} \sigma_{q A L, t}+\left(\psi_{A E, t}+\psi_{E E, t}\right) \sigma_{E L} \sigma_{q E L, t} \\
= & \psi_{A, t} \sigma_{A O} \sigma_{q A O, t}+\left(1-\psi_{A, t}\right) \sigma_{E O} \sigma_{q E O, t} \\
& +\left(\psi_{A A, t}+\psi_{E A, t}\right) \sigma_{A L} \sigma_{q A L, t}+\left(\psi_{A E, t}+\psi_{E E, t}\right) \sigma_{E L} \sigma_{q E L, t}
\end{aligned}
$$

Plugging back into the law of motion for $\eta_{t}$, we obtain

$$
\begin{aligned}
\mu_{\eta, t}= & \frac{\bar{a} P_{a, t}}{q_{t}}-\frac{C_{A, t}}{N_{A, t}}+\left(x_{A, t}-1\right)\left(\gamma_{A} \varsigma_{A A, t}+\left(\gamma_{A}-1\right) \varsigma_{\xi A, t}\right)-\left(\Sigma_{N A, t}-\Sigma_{q, t}-\Sigma_{K, t}\right) \cdot\left(\Sigma_{q, t}+\Sigma_{K, t}\right) \\
& +\left(1-\psi_{A, t}\right)\left(\sigma_{A O} \sigma_{q A O, t}-\sigma_{E O} \sigma_{q E O, t}\right)+\left(\frac{x_{A A, t}}{x_{A, t}}-\psi_{A A, t}-\psi_{E A, t}\right) \sigma_{A L} \sigma_{q A L, t} \\
& +\left(\frac{x_{A E, t}}{x_{A, t}}-\psi_{A E, t}-\psi_{E E, t}\right) \sigma_{E L} \sigma_{q E L, t}
\end{aligned}
$$


for the drift. For the volatility vector, we have

$$
\begin{aligned}
\Sigma_{\eta, t}= & {\left[\begin{array}{c}
x_{A, t}\left(\sigma_{A O}+\sigma_{q A O, t}\right)-\sigma_{q A, t}-\psi_{A, t} \sigma_{A O} \\
x_{A, t} \sigma_{q E O, t}-\sigma_{q E, t}-\left(1-\psi_{A, t}\right) \sigma_{E O} \\
x_{A A, t}\left(\sigma_{A L}+\sigma_{q A L, t}\right)-\sigma_{q A L, t}-\left(\psi_{A A, t}+\psi_{E A, t}\right) \sigma_{A L} \\
x_{A E, t}\left(\sigma_{E L}+\sigma_{q E L, t}\right)-\sigma_{q E L, t}-\left(\psi_{A E, t}+\psi_{E E, t}\right) \sigma_{E L}
\end{array}\right] } \\
= & {\left[\begin{array}{c}
\left(x_{A, t}-\psi_{A, t}\right) \sigma_{A O}+\left(x_{A, t}-1\right) \sigma_{q A, t} \\
-\left(1-\psi_{A, t}\right) \sigma_{E O}+\left(x_{A, t}-1\right) \sigma_{q E, t} \\
\left(x_{A A, t}-\psi_{A A, t}-\psi_{E A, t}\right) \sigma_{A L}+\left(x_{A A, t}-1\right) \sigma_{q A L, t} \\
\left(x_{A E, t}-\psi_{A E, t}-\psi_{E E, t}\right) \sigma_{E L}+\left(x_{A E, t}-1\right) \sigma_{q E L, t}
\end{array}\right] }
\end{aligned}
$$

We now write portfolio shares in terms of capital allocations by observing

$$
x_{A, t}=\frac{\psi_{A, t}}{\eta_{t}}, \quad x_{A A, t}=\frac{\psi_{A A, t}}{\eta_{t}}, \quad x_{A E, t}=\frac{\psi_{A E, t}}{\eta_{t}} .
$$

The drift of $\eta$ becomes

$$
\begin{aligned}
\mu_{\eta, t}= & \frac{\bar{a} P_{a, t}}{q_{t}}-\frac{C_{A, t}}{N_{A, t}}+\left(\frac{\psi_{A, t}}{\eta_{t}}-1\right)\left(\gamma_{A} \varsigma_{A a, t}+\left(\gamma_{A}-1\right) \varsigma_{\xi A, t}\right) \\
& -\left(\Sigma_{N A, t}-\Sigma_{q, t}-\Sigma_{K, t}\right) \cdot\left(\Sigma_{q, t}+\Sigma_{K, t}\right)+\left(1-\psi_{A, t}\right)\left(\sigma_{A O} \sigma_{q A O, t}-\sigma_{E O} \sigma_{q E O, t}\right) \\
& +\left(\frac{\psi_{A A, t}}{\psi_{A, t}}-\psi_{A A, t}-\psi_{E A, t}\right) \sigma_{A L} \sigma_{q A L, t}+\left(\frac{\psi_{A E, t}}{\psi_{A, t}}-\psi_{A E, t}-\psi_{E E, t}\right) \sigma_{E L} \sigma_{q E L, t}
\end{aligned}
$$

and the volatility of $\eta$ becomes

$$
\Sigma_{\eta, t}=\left[\begin{array}{c}
\psi_{A, t}\left(\frac{1}{\eta_{t}}-1\right) \sigma_{A O}+\left(\frac{\psi_{A, t}}{\eta_{t}}-1\right) \sigma_{q A O, t} \\
-\left(1-\psi_{A, t}\right) \sigma_{E O}+\left(\frac{\psi_{A, t}}{\eta_{t}}-1\right) \sigma_{q E O, t} \\
\left(\frac{\psi_{A A, t}}{\eta_{t}}-\psi_{A A, t}-\psi_{E A, t}\right) \sigma_{A L}+\left(\frac{\psi_{A A, t}}{\eta_{t}}-1\right) \sigma_{q A L, t} \\
\left(\frac{\psi_{A E, t}}{\eta_{t}}-\psi_{A E, t}-\psi_{E E, t}\right) \sigma_{E L}+\left(\frac{\psi_{A E, t}}{\eta_{t}}-1\right) \sigma_{q E L, t} .
\end{array}\right]
$$




\section{F.3 Asset Price Volatilities}

For the remainder of this appendix section, we suppress time subscripts and regard equilibrium quantities as functions of $\eta$. By Ito's lemma, for a twice-continuously differentiable $f$, the volatility vector of $f$ is

$$
\Sigma_{f}=\frac{f^{\prime}}{f} \Sigma_{\eta} \eta
$$

Thus, we have

$$
\Sigma_{q}=\left[\begin{array}{c}
\sigma_{q A O} \\
\sigma_{q E O} \\
\sigma_{q A L} \\
\sigma_{q E L}
\end{array}\right]=\frac{q^{\prime}}{q} \Sigma_{\eta} \eta=\frac{q^{\prime}}{q}\left[\begin{array}{c}
\psi_{A}(1-\eta) \sigma_{A O}+\left(\psi_{A}-\eta\right) \sigma_{q A O} \\
-\left(1-\psi_{A}\right) \eta \sigma_{E O}+\left(\psi_{A}-\eta\right) \sigma_{q E O} \\
\left(\psi_{A A}(1-\eta)-\psi_{E A} \eta\right) \sigma_{A L}+\left(\psi_{A A}-\eta\right) \sigma_{q A L} \\
\left(\psi_{A E}(1-\eta)-\psi_{E E} \eta\right) \sigma_{E L}+\left(\psi_{A E}-\eta\right) \sigma_{q E L}
\end{array}\right]
$$

Solving for the volatilities of $q$ yields

$$
\begin{aligned}
\sigma_{q A O} & =\frac{q^{\prime} \psi_{A}(1-\eta) \sigma_{A O}}{q-q^{\prime}\left(\psi_{A}-\eta\right)} \\
\sigma_{q E O} & =-\frac{q^{\prime}\left(1-\psi_{A}\right) \eta \sigma_{E O}}{q-q^{\prime}\left(\psi_{A}-\eta\right)} \\
\sigma_{q A L} & =\frac{q^{\prime}\left(\psi_{A A}(1-\eta)-\psi_{E A} \eta\right) \sigma_{A L}}{q-q^{\prime}\left(\psi_{A A}-\eta\right)} \\
\sigma_{q E L} & =\frac{q^{\prime}\left(\psi_{A E}(1-\eta)-\psi_{E E} \eta\right) \sigma_{E L}}{q-q^{\prime}\left(\psi_{A E}-\eta\right)}
\end{aligned}
$$

For $\xi$, we similarly have

$$
\begin{aligned}
& \sigma_{\xi_{A A}}=\frac{\xi^{\prime}}{\xi}\left(\left(\psi_{A A}(1-\eta)-\psi_{E A} \eta\right) \sigma_{A L}+\left(\psi_{A A}-\eta\right) \sigma_{q A L}\right) \\
& \sigma_{\xi_{A E}}=\frac{\xi^{\prime}}{\xi}\left(\left(\psi_{A E}(1-\eta)-\psi_{E E} \eta\right) \sigma_{E L}+\left(\psi_{A E}-\eta\right) \sigma_{q E L}\right)
\end{aligned}
$$


By (39), an AE agent holds capital in both the AE and the EME if and only if

$$
\begin{aligned}
& \sigma_{A L} \sigma_{q A L}-\sigma_{E L} \sigma_{q E L}-\gamma_{A}\left(\frac{\psi_{A A}}{\eta}\left(\sigma_{A L}+\sigma_{q A L}\right)^{2}-\frac{\psi_{A E}}{\eta}\left(\sigma_{E L}+\sigma_{q E L}\right)^{2}\right) \\
& =\left(\gamma_{A}-1\right) \frac{\xi^{\prime}}{\xi}\left(\left(\psi_{A A}(1-\eta)-\psi_{E A} \eta\right) \sigma_{A L}+\left(\psi_{A A}-\eta\right) \sigma_{q A L}\right)\left(\sigma_{A L}+\sigma_{q A L}\right) \\
& -\left(\gamma_{A}-1\right) \frac{\xi^{\prime}}{\xi}\left(\left(\psi_{A E}(1-\eta)-\psi_{E E} \eta\right) \sigma_{E L}+\left(\psi_{A E}-\eta\right) \sigma_{q E L}\right)\left(\sigma_{E L}+\sigma_{q E L}\right) .
\end{aligned}
$$

\section{F.4 Equal Volatilities for Location Shocks}

In the case that $\sigma_{A L}=\sigma_{E L}$, it is straightforward to characterize the location of capital usage. Intuitively, since the volatility of capital shocks is the same in either country, agents will split their capital usage between each country equally. In other words, $\psi_{A A}=\psi_{A E}$ and $\psi_{E A}=\psi_{E E}$. These allocations imply $\sigma_{q A L}=\sigma_{q E L}$, which delivers the following equalities

$$
\begin{aligned}
\sigma_{A L} \sigma_{q A L} & =\sigma_{E L} \sigma_{q E L} \\
\frac{\psi_{A A}}{\eta}\left(\sigma_{A L}+\sigma_{q A L}\right)^{2} & =\frac{\psi_{A E}}{\eta}\left(\sigma_{E L}+\sigma_{q E L}\right)^{2} \\
\left(\psi_{A A}(1-\eta)-\psi_{E A} \eta\right) \sigma_{A L}\left(\sigma_{A L}+\sigma_{q A L}\right) & =\left(\psi_{A E}(1-\eta)-\psi_{E E} \eta\right) \sigma_{E L}\left(\sigma_{E L}+\sigma_{q E L}\right) \\
\left(\psi_{A A}-\eta\right) \sigma_{q A L}\left(\sigma_{A L}+\sigma_{q A L}\right) & =\left(\psi_{A E}-\eta\right) \sigma_{q E L}\left(\sigma_{E L}+\sigma_{q E L}\right) .
\end{aligned}
$$

These equalities imply that the asset-pricing condition pinning down $\psi_{A A}$ is satisfied for AE agents. Thus, with equal location volatilities and allocation of capital across countries, AE agents satisfy their asset-pricing conditions. By symmery, the same reasoning applies to EME agents.

\section{F.5 Isomorphism}

In this section, we establish an observational equivalence between the baseline model and this extension when $\gamma_{A}=1$ and $\gamma_{B}=1$, i.e. both AE and EME agents have log utility. By an observational equivalence, we mean there exists parametrizations such that the models produce the same equilibrium allocations.

By symmetry of this special case, we only need to consider the problem for AE agents. Define 
$\varsigma_{A a}^{\prime}$ as the original model's parallel to $\varsigma_{A a}$. Note that since $\gamma_{A}=1$, we do not need to consider $\varsigma_{\xi_{A}}$. For this section, we will assume $\sigma_{L} \equiv \sigma_{A L}=\sigma_{E L}$ and $\sigma_{O} \equiv \sigma_{A O}=\sigma_{E O}$. We want to find $\sigma_{O}$ and $\sigma_{L}$ such that $\varsigma_{A a}^{\prime}=\varsigma_{A a}$. For reference,

$$
\zeta_{A a}^{\prime}=\frac{\psi_{A}}{\eta}\left(\left(\sigma+\sigma_{q A}\right)^{2}+\sigma_{q E}^{2}\right)
$$

We want

$$
\begin{aligned}
& \left(\sigma+\frac{q^{\prime} \psi_{A}(1-\eta) \sigma}{q-q^{\prime}\left(\psi_{A}-\eta\right)}\right)^{2}+\left(\frac{q^{\prime}\left(1-\psi_{A}\right) \eta \sigma}{q-q^{\prime}\left(\psi_{A}-\eta\right)}\right)^{2} \\
& =\left(\sigma_{O}+\frac{q^{\prime} \psi_{A}(1-\eta) \sigma_{O}}{q-q^{\prime}\left(\psi_{A}-\eta\right)}\right)^{2}+\frac{1}{2}\left(\sigma_{L}+\frac{1}{2} \frac{q^{\prime}\left(\psi_{A}(1-\eta)-\psi_{E} \eta\right) \sigma_{L}}{q-q^{\prime}\left(\psi_{A} / 2-\eta\right)}\right)^{2} \\
& +\left(\frac{q^{\prime}\left(1-\psi_{A}\right) \eta \sigma_{O}}{q-q^{\prime}\left(\psi_{A}-\eta\right)}\right)^{2} \\
& 2\left(\sigma+\frac{q^{\prime} \psi_{A}(1-\eta) \sigma}{q-q^{\prime}\left(\psi_{A}-\eta\right)}\right)^{2}-2\left(\sigma_{O}+\frac{q^{\prime} \psi_{A}(1-\eta) \sigma_{O}}{q-q^{\prime}\left(\psi_{A}-\eta\right)}\right)^{2} \\
& +2\left(\frac{q^{\prime}\left(1-\psi_{A}\right) \eta \sigma}{q-q^{\prime}\left(\psi_{A}-\eta\right)}\right)^{2}-2\left(\frac{q^{\prime}\left(1-\psi_{A}\right) \eta \sigma_{O}}{q-q^{\prime}\left(\psi_{A}-\eta\right)}\right)^{2} \\
& =\left(\sigma_{L}+\frac{1}{2} \frac{q^{\prime}\left(\psi_{A}(1-\eta)-\psi_{E} \eta\right) \sigma_{L}}{q-q^{\prime}\left(\psi_{A} / 2-\eta\right)}\right)^{2} \\
& 2\left[\left(\sigma^{2}-\sigma_{O}^{2}\right)+2 \frac{q^{\prime} \psi_{A}(1-\eta)}{q-q^{\prime}\left(\psi_{A}-\eta\right)}\left(\sigma^{2}-\sigma_{O}\right)^{2}+\left(\frac{q^{\prime} \psi_{A}(1-\eta)}{q-q^{\prime}\left(\psi_{A}-\eta\right)}\right)^{2}\left(\sigma^{2}-\sigma_{O}^{2}\right)\right] \\
& +2\left(\frac{q^{\prime}\left(1-\psi_{A}\right) \eta}{q-q^{\prime}\left(\psi_{A}-\eta\right)}\right)^{2}\left(\sigma^{2}-\sigma_{O}^{2}\right) \\
& =\sigma_{L}^{2}+\frac{q^{\prime}\left(\psi_{A}(1-\eta)-\left(1-\psi_{A}\right) \eta\right)}{q-q^{\prime}\left(\psi_{A} / 2-\eta\right)} \sigma_{L}^{2}+\frac{1}{4}\left(\frac{q^{\prime}\left(\psi_{A}(1-\eta)-\left(1-\psi_{A}\right) \eta\right.}{q-q^{\prime}\left(\psi_{A} / 2-\eta\right)}\right)^{2} \sigma_{L}^{2} \\
& 2\left(\sigma^{2}-\sigma_{O}^{2}\right)\left[1+2 \frac{q^{\prime}\left(\psi_{A}(1-\eta)\right)}{q-q^{\prime}\left(\psi_{A}-\eta\right)}+\left(\frac{q^{\prime}\left(\psi_{A}(1-\eta)\right)}{q-q^{\prime}\left(\psi_{A}-\eta\right)}\right)^{2}+\left(\frac{q^{\prime}\left(1-\psi_{A}\right) \eta}{q-q^{\prime}\left(\psi_{A}-\eta\right)}\right)^{2}\right] \\
& =2\left(\sigma^{2}-\sigma_{O}^{2}\right)\left[\left(1+\frac{q^{\prime}\left(\psi_{A}(1-\eta)\right)}{q-q^{\prime}\left(\psi_{A}-\eta\right)}\right)^{2}+\left(\frac{q^{\prime}\left(1-\psi_{A}\right) \eta}{q-q^{\prime}\left(\psi_{A}-\eta\right)}\right)^{2}\right] \\
& =\sigma_{L}^{2}\left(1+\frac{1}{2} \frac{q^{\prime}\left(\psi_{A}(1-\eta)-\left(1-\psi_{A}\right) \eta\right.}{q-q^{\prime}\left(\psi_{A} / 2-\eta\right)}\right)^{2} \text {. }
\end{aligned}
$$


Re-arranging obtains

$$
\begin{aligned}
& \sigma_{L}^{2}=2\left(\sigma^{2}-\sigma_{O}^{2}\right) \frac{\left(1+\frac{q^{\prime}\left(\psi_{A}(1-\eta)\right)}{q-q^{\prime}\left(\psi_{A}-\eta\right)}\right)^{2}+\left(\frac{q^{\prime}\left(1-\psi_{A}\right) \eta}{q-q^{\prime}\left(\psi_{A}-\eta\right)}\right)^{2}}{\left(1+\frac{1}{2} \frac{q^{\prime}\left(\psi_{A}(1-\eta)-\left(1-\psi_{A}\right) \eta\right)}{q-q^{\prime}\left(\psi_{A} / 2-\eta\right)}\right)^{2}} \\
& \sigma_{L}^{2}=2\left(\sigma^{2}-\sigma_{O}^{2}\right) \frac{\left(\frac{q-q^{\prime}\left(1-\psi_{A}\right) \eta}{q-q^{\prime}\left(\psi_{A}-\eta\right)}\right)^{2}+\left(\frac{q^{\prime}\left(1-\psi_{A}\right) \eta}{q-q^{\prime}\left(\psi_{A}-\eta\right)}\right)^{2}}{\left(1+\frac{1}{2} \frac{q^{\prime}\left(\psi_{A}-\eta\right)}{q-q^{\prime}\left(\psi_{A} / 2-\eta\right)}\right)^{2}} \\
& \sigma_{L}^{2}=2\left(\sigma^{2}-\sigma_{O}^{2}\right) \frac{\frac{q^{2}-2 q q^{\prime}\left(1-\psi_{A}\right) \eta+\left(q^{\prime}\left(1-\psi_{A}\right) \eta\right)^{2}+\left(q^{\prime}\left(1-\psi_{A}\right) \eta\right)^{2}}{\left(q-q^{\prime}\left(\psi_{A}-\eta\right)\right)^{2}}}{\left(1+\frac{1}{2} \frac{q^{\prime}\left(\psi_{A}-\eta\right)}{q-q^{\prime}\left(\psi_{A} / 2-\eta\right)}\right)^{2}} \\
& \sigma_{L}^{2}=2\left(\sigma^{2}-\sigma_{O}^{2}\right) \frac{q^{2}-2 q q^{\prime}\left(1-\psi_{A}\right) \eta+2\left(q^{\prime}\left(1-\psi_{A}\right) \eta\right)^{2}}{\left(q-q^{\prime}\left(\psi_{A}-\eta\right)\right)^{2}\left(1+\frac{1}{2} \frac{q^{\prime}\left(\psi_{A}-\eta\right)}{q-q^{\prime}\left(\psi_{A} / 2-\eta\right)}\right)^{2}}
\end{aligned}
$$

Thus, for a given $q, q^{\prime}$, and $\psi_{A}$, we can obtain $\varsigma_{A a}^{\prime}=\varsigma_{A a}$ if we choose $\sigma_{L}$ and $\sigma_{O}$ such that $\sigma_{O}<\sigma$ and they satisfy

$$
\sigma_{L}=\sqrt{2\left(\sigma^{2}-\sigma_{O}^{2}\right)} \frac{\sqrt{q^{2}-2 q q^{\prime}\left(1-\psi_{A}\right) \eta+2\left(q^{\prime}\left(1-\psi_{A}\right) \eta\right)^{2}}}{\left|\left(q-q^{\prime}\left(\psi_{A}-\eta\right)\right)\left(1+\frac{1}{2} \frac{q^{\prime}\left(\psi_{A}-\eta\right)}{q-q^{\prime}\left(\psi_{A} / 2-\eta\right)}\right)\right|} .
$$

We now have equivalent risk premia in the baseline model and in this extension for AE and EME agents. Since agents have unit EIS in the baseline model as well, the two equations pinning down the behavior of $q$ and $\psi_{A}$ are market clearing for consumption and the difference between the AE's asset-pricing condition and the EME's asset-pricing condition. Because risk premia are the same when $\sigma_{O}$ and $\sigma_{L}$ satisfy the above conditions, $q$ and $\psi_{A}$ solve the same equations as in the baseline model. Therefore, the extension of the baseline model is observationally equivalent in the sense that capital allocations and prices are equivalent. 


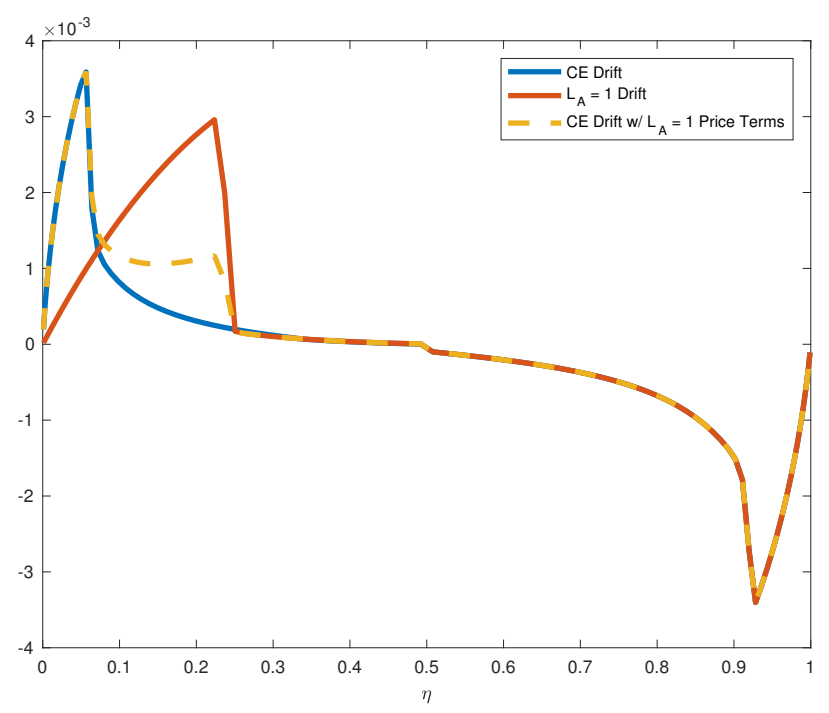

(a) CE Drift

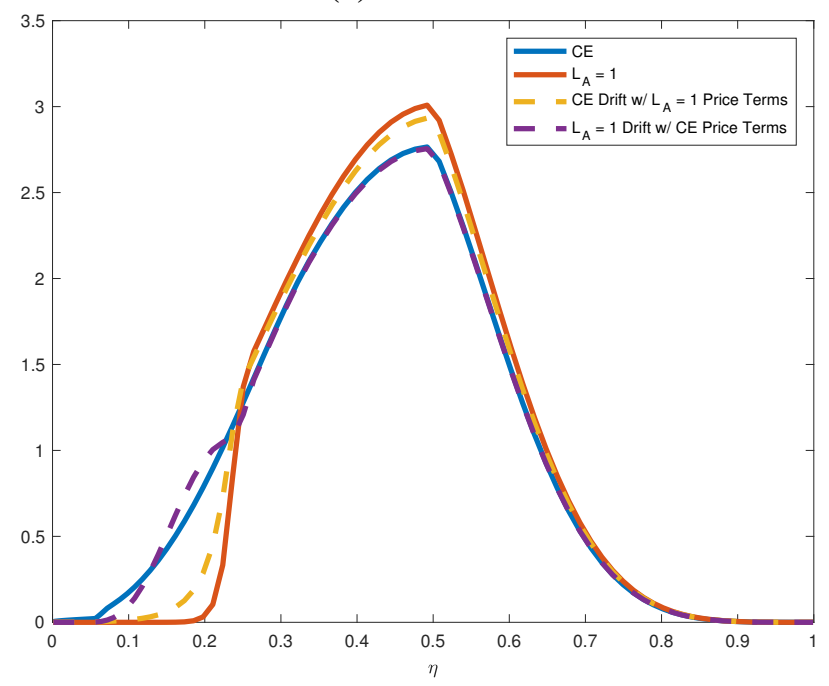

(c) Counterfactual Drifts

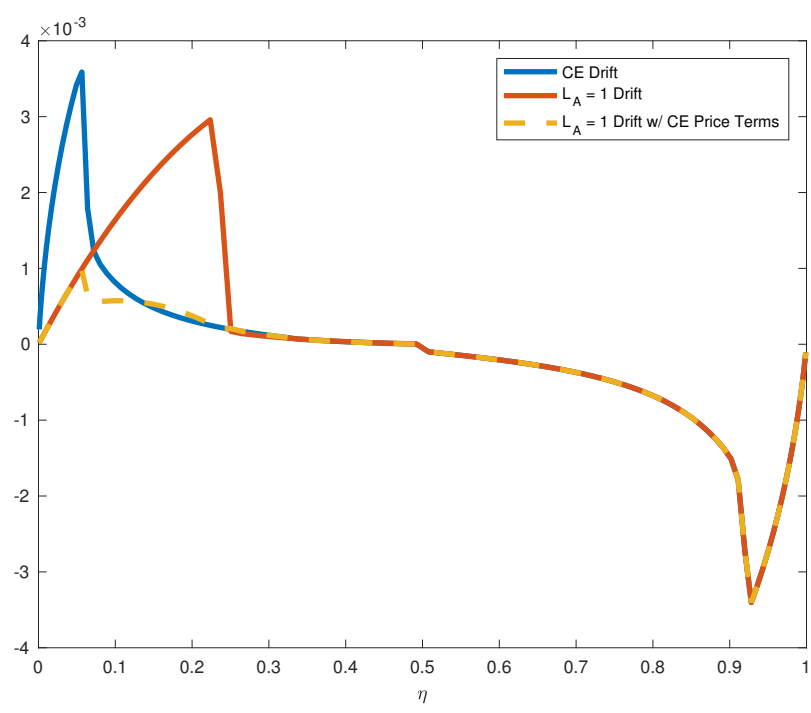

(b) $L_{A}$ Drift

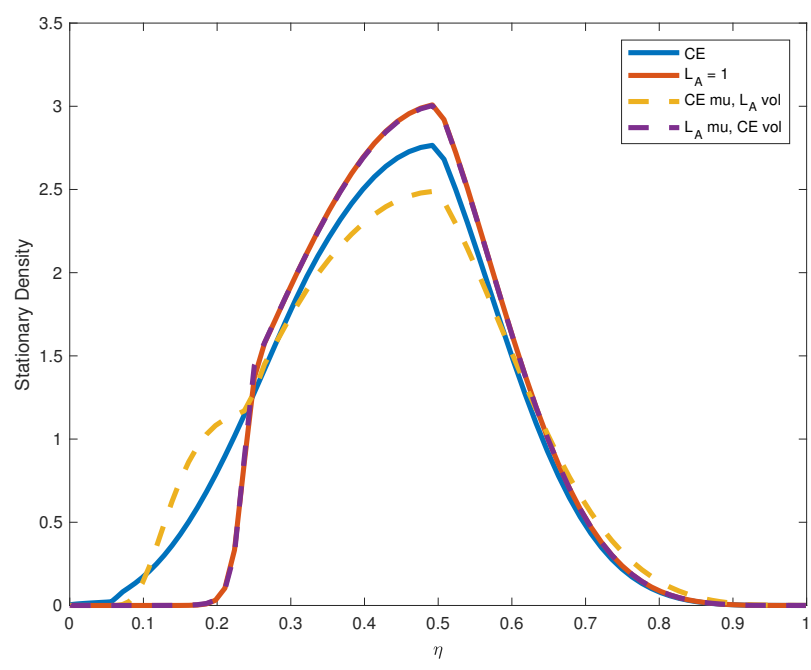

(d) Counterfactual Drift and Volatility Pairs

Figure 12: This figure examines counterfactual $\mu_{\eta}$ and stationary distribution. In all cases, $L_{E}$ does not bind. Panels (a) and (b) plot $\mu_{\eta}$ from the competitive equilibrium with the solid blue line and from the $L_{A}=1$ equilibrium with the solid red line. The yellow dashed line in (a) plots $\mu_{\eta}$ from the competitive equilibrium but with the prices of intermediate goods that appear in the $\mu_{\eta}$ term replaced by the prices from the $L_{A}=1$ equilibrium. The yellow dashed line in (b) plots $\mu_{\eta}$ from the $L_{A}=1$ equilibrium but with the prices of intermediate goods replaced by the prices from the competitive equilibrium. Panels (c) and (d) plots the stationary distributions from the competitive equilibrium and the $L_{A}=1$ equilibrium in solid blue and red lines, respectively. Panel (c) also plots the distributions implied by the counterfactual drifts from Panels (a) and (b). The dashed yellow line in Panel $(\mathrm{d})$ is the counterfactual stationary distribution if we use the drift from the competitive equilibrium paired with the volatility from the $L_{A}=1$ equilibrium. The dashed purple line in Panel (d) is the stationary distribution if we use the drift from the $L_{A}=1$ equilibrium paired with the volatility from the competitive equilibrium. 